\title{
Monte Carlo Studies of the ATLAS Forward Calorimeter and of the Supersymmetric Top Quark
}

\author{
by
}

\section{John Paul Archambault}

\author{
A thesis submitted to the \\ Faculty of Graduate Studies and Research \\ in partial fulfillment of the requirements \\ for the degree of
}

\section{Doctor of Philosophy}

\author{
Department of Physics \\ Carleton University \\ Ottawa-Carleton Institute of Physics \\ Ottawa, Canada
}

February 25, 2009

Copyright (C) 2009 John Paul Archambault 
Library and

Archives Canada

Published Heritage

Branch

395 Wellington Street

Ottawa ON K1A 0N4

Canada
Bibliothèque et

Archives Canada

Direction du

Patrimoine de l'édition

395 , rue Wellington

Ottawa ON K1A 0N4

Canada

Your file Votre référence

ISBN: 978-0-494-52058-1

Ourfile Notre référence

ISBN: 978-0-494-52058-1

NOTICE:

The author has granted a nonexclusive license allowing Library and Archives Canada to reproduce, publish, archive, preserve, conserve, communicate to the public by telecommunication or on the Internet, loan, distribute and sell theses worldwide, for commercial or noncommercial purposes, in microform, paper, electronic and/or any other formats.

The author retains copyright ownership and moral rights in this thesis. Neither the thesis nor substantial extracts from it may be printed or otherwise reproduced without the author's permission.
AVIS:

L'auteur a accordé une licence non exclusive permettant à la Bibliothèque et Archives Canada de reproduire, publier, archiver, sauvegarder, conserver, transmettre au public par télécommunication ou par l'Internet, prêter, distribuer et vendre des thèses partout dans le monde, à des fins commerciales ou autres, sur support microforme, papier, électronique et/ou autres formats.

L'auteur conserve la propriété du droit d'auteur et des droits moraux qui protège cette thèse. $\mathrm{Ni}$ la thèse ni des extraits substantiels de celle-ci ne doivent être imprimés ou autrement reproduits sans son autorisation.
In compliance with the Canadian

Privacy Act some supporting forms may have been removed from this thesis.

While these forms may be included in the document page count, their removal does not represent any loss of content from the thesis.
Conformément à la loi canadienne sur la protection de la vie privée, quelques formulaires secondaires ont été enlevés de cette thèse.

Bien que ces formulaires aient inclus dans la pagination, il n'y aura aucun contenu manquant.

\section{Canadä}




\section{Abstract}

The Large Hadron Collider will collide $7 \mathrm{TeV}$ proton beams with the intent of studying the Standard Model of Particle Physics and searching for physics beyond. One of the general purpose detectors designed to study the high energy proton collisions is called the ATLAS detector.

The Liquid Argon Forward Calorimeter (FCal) of the ATLAS detector is an important component for the studies mentioned above. The FCal contains both electromagnetic and hadronic modules and in 2003 , a beam test was conducted by the ATLAS FCal group to acquire data for the energy calibration of the FCal modules. Using the $\mathrm{H} 6$ beam line at CERN, the beam test was performed to investigate the response of the FCal to both electrons and pions in the energy range of (10-200) GeV. In the context of this thesis work, a simulation of the beam test was incorporated into the ATLAS software framework to study the beam test data. This thesis presents results of the Geant 4 simulation, which are compared to the data to quantify the performance of the FCal over the above mentioned energy range.

One of the most promising extensions of the Standard Model is known as Supersymmetry and it will be a major focus of the ATLAS experiment. In supersymmetric theories, each Standard Model particle has a superpartner differing in its spin statistics. A light supersymmetric partner of the top quark (stop) is motivated by theories of electroweak baryogenesis in the Minimal Supersymmetric Standard Model (MSSM). This thesis takes a phenomenological study of an MSSM benchmark point, LST1, with a stop mass of $150 \mathrm{GeV}$, and investigates the discovery sensitivity with the ATLAS detector. 


\section{Acknowledgements}

First of all, I would like to thank my supervisor, Manuella. Her dedication to physics and to her students is unmatched. I could not have asked for a better supervisor.

I would also like to thank Gerald Oakham, the team leader of the Carleton ATLAS group. The generosity of the group has provided me with a number of opportunities and experiences that I will never forget. The rest of the Carleton ATLAS group has also been instrumental in my projects, providing feedback at group meetings as well as through informal discussions. Throughout my years at Carleton the group has evolved, including members D. Asner, C. Cojocaru, L. Heelan, M. Khakzad, C. Liu, M. Schram, Y.H. Shin, Z. Yang and most recently T. McCarthy, A. Robichaud, R. Ueno and K. Whalen.

Of these members, I worked mostly with Mohsen Khakzad, Louise Heelan and Zhaoyu Yang. I truly enjoyed our time working together. Thank you for taking the time to work with me.

Over the years, I have also received help from people in the ATLAS Canada community as well as in the ATLAS FCal group.

Thank you to all my friends and family. Even though it seemed that your favourite question was "When will you be finished?", I appreciate your interest in my endeavours!

Last, but not least, thank you to Mom, Dad, Mike and Jen. Your continued support has meant everything to me. 


\section{Contents}

1 Introduction 1

1.1 The Standard Model and Beyond . . . . . . . . . . . . . 1

2 The LHC and ATLAS 9

2.1 The Large Hadron Collider . . . . . . . . . . . . . . . . . . 9

2.1.1 The Accelerator ................. 10

2.1 .2 Proton-Proton Interactions . . . . . . . . . . . . 12

2.2 The ATLAS Experiment . . . . . . . . . . . . 15

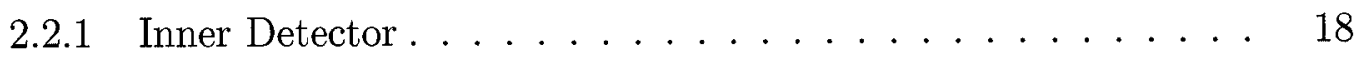

2.2 .2 Calorimeters........................ 19

2.2.3 Muon Spectrometer . . . . . . . . . . . . . 22

2.2 .4 Trigger ........................... 24

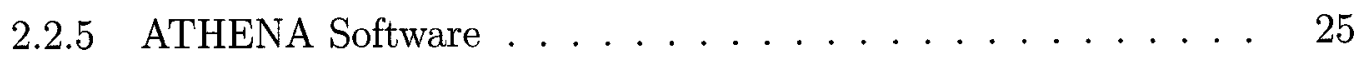

3 The ATLAS Forward Calorimeter $\quad 29$ 
3.1 Calorimetry . . . . . . . . . . . . . . . . . 29

3.1.1 Electromagnetic Showers . . . . . . . . . . . . . . 30

3.1 .2 Hadronic Showers . . . . . . . . . . . . . . . . . . 33

3.1 .3 Sampling Calorimeters . . . . . . . . . . . . 36

3.2 The ATLAS Forward Calorimeter . . . . . . . . . . . . 39

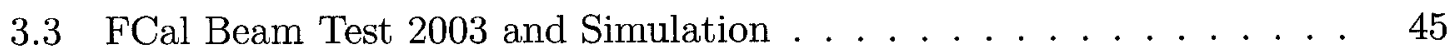

3.3.1 Simulation of the FCal Modules . . . . . . . . . . . 53

3.3.2 Geant4 Physics Lists . . . . . . . . . . . . . . . . 54

3.3.3 FCal Simulation Performance $\ldots \ldots \ldots \ldots \ldots$

3.4 Summary . . . . . . . . . . . . . . . . . 64

4 Studies of a Light Stop with the ATLAS Detector 66

4.1 Supersymmetry . . . . . . . . . . . . . . 67

4.2 Light Stops . . . . . . . . . . . . . . . . . . . 73

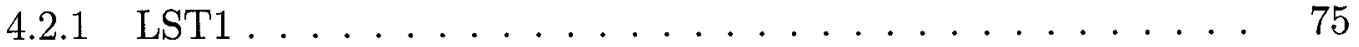

4.2.2 Light Stop Signature at the LHC $\ldots \ldots \ldots \ldots$

4.2 .3 Monte Carlo Production . . . . . . . . . . . . . . 79

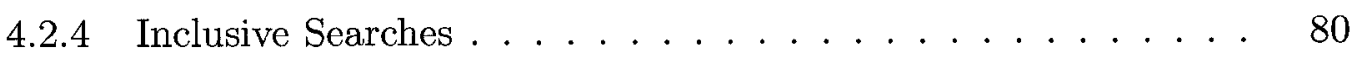

4.2 .5 Exclusive Searches . . . . . . . . . . . . . . 81

4.3 Summary $\ldots \ldots \ldots \ldots \ldots \ldots \ldots \ldots \ldots \ldots \ldots \ldots$ 
$\begin{array}{ll}\text { A Collider Physics Parameters } & 97\end{array}$

B ATLFAST Physics Objects $\quad 99$

B.1 Reconstruction of Physics Objects in ATLFAST . . . . . . . . . . . 99

C Invariant Mass Distribution Formulas 105

D Projects 


\section{List of Tables}

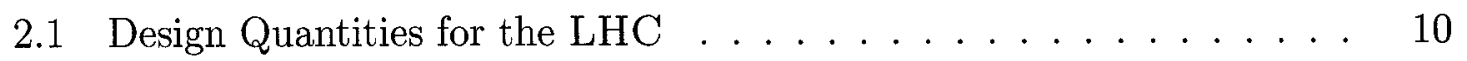

3.1 Properties of the ATLAS FCal Modules . . . . . . . . . . . . 44

3.2 Cryostat Positions $\ldots \ldots \ldots \ldots \ldots \ldots \ldots \ldots \ldots$

3.3 Simulated Material Properties for FCal Hadronic Modules . . . . . 54

$3.4 \quad$ FCal Sampling Fractions . . . . . . . . . . . . . . . 58

$3.5 \quad$ FCal Linearity, Energy Resolution . . . . . . . . . . . . . . 62

4.1 MSSM Chiral Supermultiplets $\ldots \ldots \ldots \ldots \ldots$

4.2 MSSM Vector Supermultiplet $\ldots \ldots \ldots \ldots \ldots$

$4.3 \quad$ LST1 Mass Spectrum . . . . . . . . . . . . . . . . . . 77

4.4 Gluino-Pair Cross-Section $\ldots \ldots \ldots \ldots \ldots$

4.5 Signal and Background Cut Flow $\ldots \ldots \ldots \ldots \ldots$

4.6 Cut Flow for Different Gluino Masses . . . . . . . . . . . . 83

4.7 Invariant Mass Fit Results . . . . . . . . . . . . . 87

viii 
4.8 Systematic Uncertainties: Selection Criteria . . . . . . . . . . . 91

4.9 Systematic Uncertainties: Invariant Mass Parameters . . . . . . . . 92 


\section{List of Figures}

1.1 Unification . . . . . . . . . . . . . . . 2

1.2 Standard Model . . . . . . . . . . . . . . . . 3

2.1 The CERN Accelerator Complex . . . . . . . . . . 11

2.2 Proton-Proton Collisions . . . . . . . . . . . . . . 13

2.3 Proton-Proton Cross-Sections . . . . . . . . . . . . . . 14

2.4 The ATLAS Detector ..................... 15

2.5 List of the ATLAS Detector Components . . . . . . . . . 16

2.6 The Inner Detector . . . . . . . . . . . . . . . . . 19

2.7 The ATLAS Calorimeters . . . . . . . . . . . . 20

2.8 The EMEC, Tile Barrel and HEC . . . . . . . . . . . . . . . 21

2.9 The Muon Spectrometer ... . . . . . . . . . . . 23

2.10 Analysis Software Chain . . . . . . . . . . . . 26

3.1 Photon Cross-Sections in Matter ... . . . . . . . . . 31 
3.2 Energy Loss of Electrons . . . . . . . . . . . . . . . . . 32

3.3 Particle Showers . . . . . . . . . . . . . . . 34

3.4 Liquid Argon Electrode . . . . . . . . . . . . . . . 37

3.5 Schematic Diagram of the Forward Calorimeter . . . . . . . . . 41

3.6 FCal Geometry Structures . . . . . . . . . . . . . . . . . . 42

3.7 Schematic Diagram of FCal2,3 f . . . . . . . . . . . . 45

3.8 Beam Spot Positions . . . . . . . . . . . . . . . . . 47

3.9 Schematic Diagram for the 2003 Beam Test . . . . . . . . . 48

3.10 Schematic Diagram of the Test Beam Cryostat . . . . . . . . . 49

3.11 FCal Beam Test Readout Coverage . . . . . . . . . . . . . . . 51

3.12 Energy Deposition in FCall . . . . . . . . . . . . . . 52

3.13 FCal Sampling Fraction Geometry Setup . . . . . . . . . . . 56

3.14 FCal Electron Sampling Fractions . . . . . . . . . . . . . . 57

3.15 Electron Energy Distributions . . . . . . . . . . . . 60

3.16 Linearity and Energy Residuals for Electrons . . . . . . . . . . . . 61

3.17 Energy Resolution for Electrons . . . . . . . . . . . . . 63

4.1 Gauge Couplings . . . . . . . . . . . . . . . 72

4.2 Stop Mass Limits . . . . . . . . . . . . . . . . . . 75

$4.3 \mathrm{LST} 1 \mathrm{Input} \operatorname{Spectrum} \ldots \ldots \ldots \ldots$. . . . . . . . . . . . . . . . .

4.4 Gluino Pair Decay Chain . . . . . . . . . . . . . . 79 
4.5 Effective Mass . . . . . . . . . . . . . . . . . 81

4.6 LST1 Significance . . . . . . . . . . . . . . . . 84

4.7 Invariant Mass Distributions for $M_{b c}$ and $M_{l c} \ldots \ldots . \ldots$

4.8 Comparison of Invariant Masses . . . . . . . . . . . . . . . . . 88

4.9 Correction Function . . . . . . . . . . . . . . . . . . . 90 


\section{Chapter 1}

\section{Introduction}

\subsection{The Standard Model and Beyond}

The ultimate goal in the study of particle physics is to understand how elementary particles in nature interact, with the quest for the unification of the fundamental forces providing the primary motivating factor. The known forces of nature are illustrated in Figure 1.1, as a function of energy. Electromagnetism, along with the weak nuclear force (responsible for radioactive decay), the strong nuclear force (responsible for the binding of quarks within nuclei), and gravity, comprise the four fundamental forces. The diagram illustrates the belief that all forces unify at higher energy scales.

The first forces to be unified were electricity and magnetism [1]. Electromagnetism was further unified with the weak nuclear force [2-4] to form the electroweak interaction at energies on the order of $10^{2} \mathrm{GeV}$. The theory containing the electroweak interaction and the strong interaction (also known as Quantum Chromodynamics (QCD)), is referred to as the Standard Model [5] and it is the most successful theory 


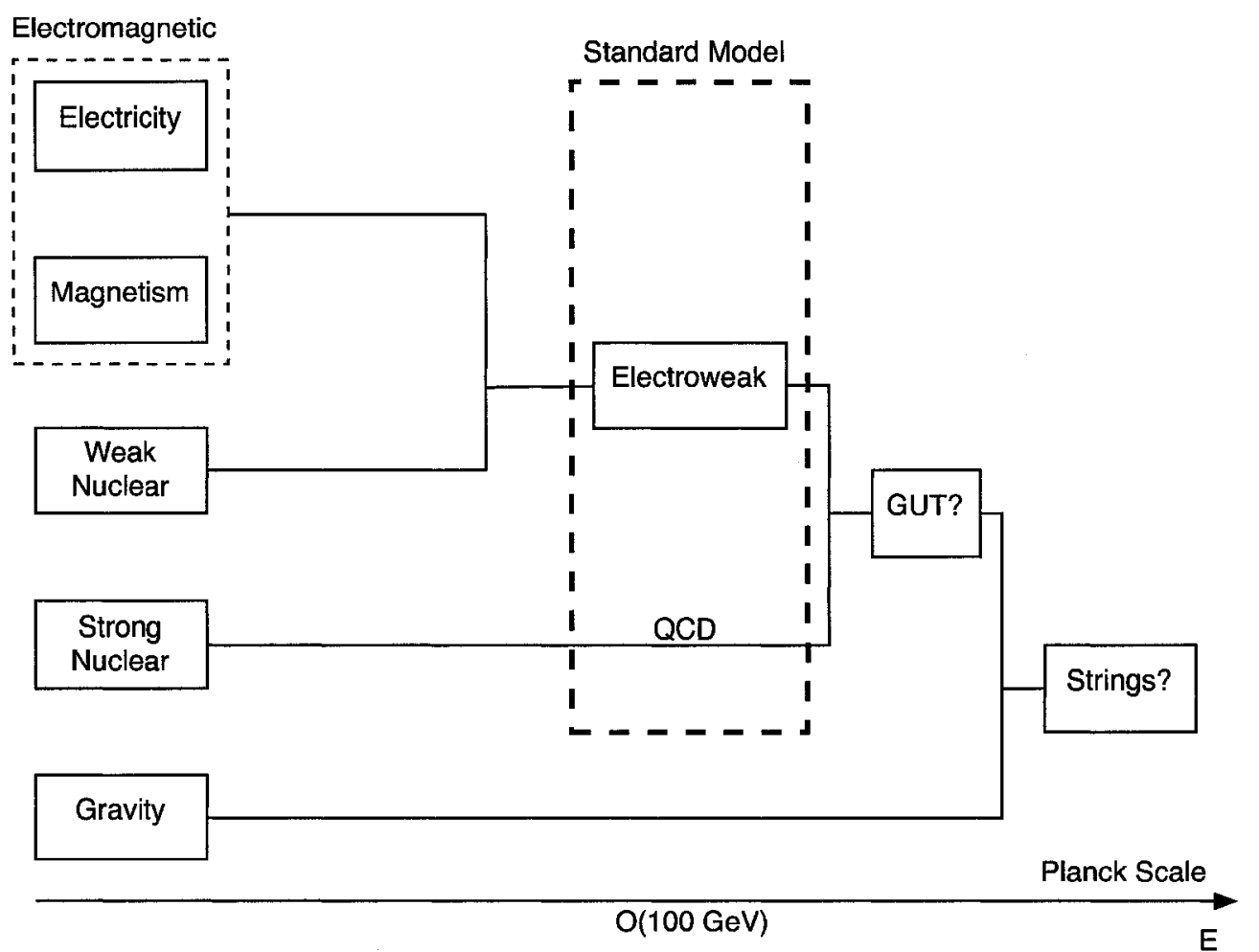

Figure 1.1: Unification of the fundamental forces.

to date in explaining elementary particles and their interactions. Theories exist in which attempts are made to unify the electroweak and strong interactions in models, known as Grand Unified Theories (GUT), occurring typically at energies of $10^{16} \mathrm{GeV}$. At even higher energies, known as the Planck Scale $\left(10^{19} \mathrm{GeV}\right)$, gravitational effects become important. It is at this energy scale where string theory may provide answers in the quest to unify gravity with the other forces.

The Standard Model is composed of two different types of particles called fermions and bosons, illustrated in Figure $1.2^{1}$. The fermions have 1/2-integer spin values, while the bosons have integer spins. The vector (spin-1) bosons propagate the

\footnotetext{
${ }^{1}$ The convention $\hbar=c=1$ is used. Therefore, all masses, momenta and energies are quoted with dimensions of energy.
} 


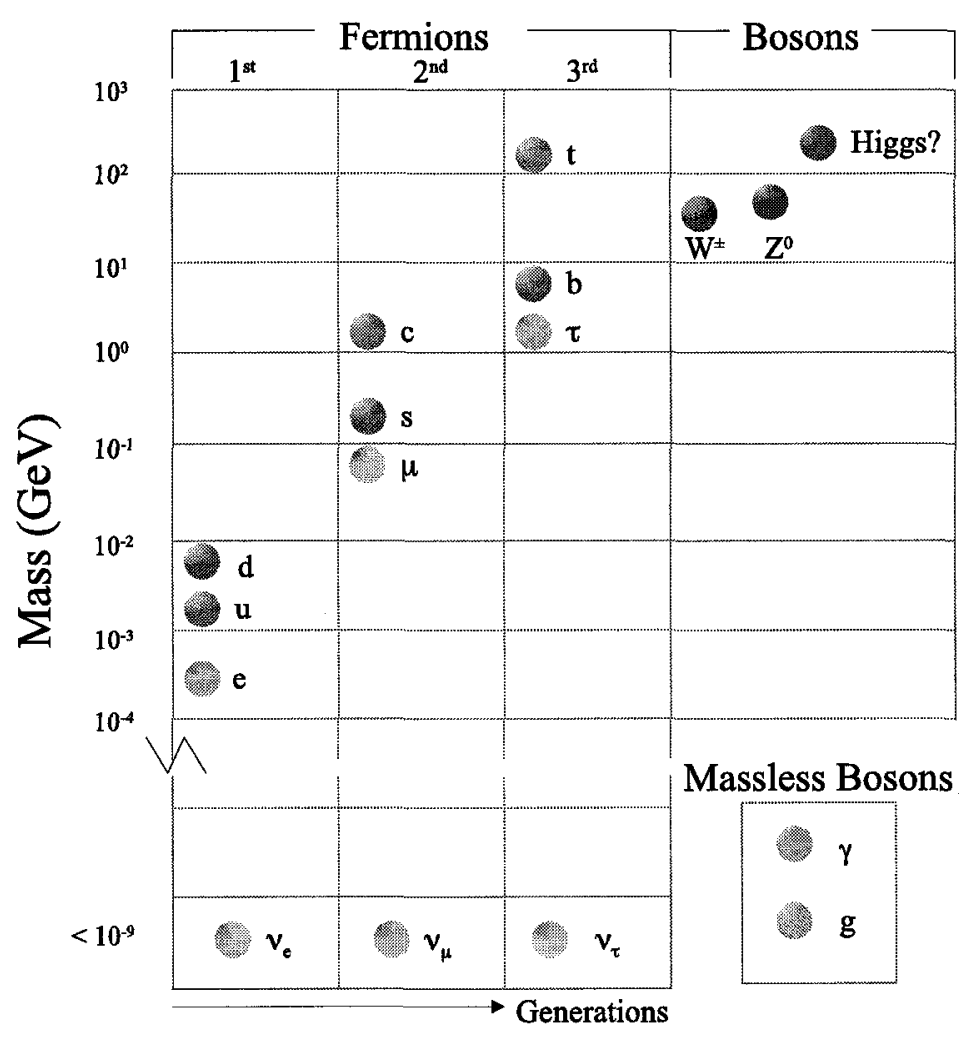

Figure 1.2: Particle content of the Standard Model [6].

forces (interactions) in the Standard Model. The photon $(\gamma)$ is responsible for the electromagnetic interaction, the $W^{ \pm}$and the $Z^{0}$ mediate the weak interaction and the gluons $(g)$ propagate the strong interaction.

The fermions make up the matter of the universe and are further subdivided into two categories known as leptons and quarks. The leptons are charged under the electroweak interaction while the quarks are charged under both the electroweak and strong interactions. The Standard Model contains three families of both quarks and leptons, along with their anti-particles. The leptons include the electron $(e)$, the 
muon $(\mu)$, and the tau $(\tau)$ with corresponding neutrinos $\left(\nu_{e}\right),\left(\nu_{e}\right)$ and $\left(\nu_{\tau}\right)^{2}$. The three families of quarks consist of the up and down $(u, d)$, the charm and strange $(c, s)$ and the top and bottom $(\mathrm{t}, \mathrm{b})$ quarks.

The Standard Model is a quantum field theory built on gauge theories, reflecting the underlying local gauge symmetries that are present. It is composed of three gauge theories corresponding to the three forces present in the theory. By requiring local gauge invariance in the Lagrangian densities for the fermion fields, interactions between the fermion field and the gauge field occur. This was first discovered in quantum electrodynamics (QED) [9-12], where local gauge invariance of the electron field requires the existence of the photon.

The success of QED led to the construction of gauge theories for the electroweak and the strong interactions. The resulting gauge group for the Standard Model is the product $S U(3)_{c} \times S U(2)_{L} \times U(1)_{Y}$ corresponding to the colour, the weak and the hypercharge symmetries, respectively. The ' $\mathrm{L}$ ' in the weak gauge group signifies that only the chiral-left components of the fermions are involved in the charged weak interaction. For this reason, the left-handed fermions are arranged in doublets while the right-handed fermions are singlets under the $S U(2)_{L}$ gauge group.

A major difference in $\mathrm{QCD}$ is that, unlike the photon which is electromagnetically neutral, the gluons are charged under colour. This is believed to be the cause of confinement, the phenomenon where colour charges cannot be isolated. Although there is plenty of experimental evidence for confinement, an analytic proof does not currently exist. The gluon colour charge also leads to self-interactions and as a consequence the strong interaction exhibits asymptotic freedom $[13,14]$. The decrease

\footnotetext{
${ }^{2}$ The neutrinos are considered massless in the Standard Model, but recent results [7,8] prove that the neutrinos are massive particles.
} 
in the coupling strength permits the use of perturbative techniques when calculating interactions in QCD at high energies $(>>1 \mathrm{GeV})$.

One of the most important questions that remains regarding the Standard Model concerns the breaking of the electroweak symmetry and the origin of mass. In theories requiring gauge invariance, mass terms for vector fields violate the gauge symmetry, resulting in massless vector bosons, as in the case of the photon in QED. Mass terms for fermions are also forbidden in the electroweak theory, due to the fact that the left- and right-handed components transform differently under $S U(2)_{L}$. In both cases, however, massive particles exist. The $W^{ \pm}$and $Z^{0}$ have masses of $(80.389 \pm 0.025) \mathrm{GeV}$ and $(91.1875 \pm 0.0021) \mathrm{GeV}$, respectively, while the fermion masses range from $0.5 \mathrm{MeV}$ to $175 \mathrm{GeV}[6]$.

One way to solve the mass problem is to spontaneously break the local $S U(2)_{L} \times U(1)_{Y}$ gauge symmetry $[15,16]$. The process by which the symmetry is spontaneously broken is known as the Higgs mechanism $[15,17]$ and it provides a way to generate masses for both the weak gauge bosons $\left(W^{ \pm}, Z^{0}\right)$ as well as for the quarks and charged leptons, while keeping the photon massless. Furthermore, the Higgs mechanism predicts the relationship between the masses of the $W^{ \pm}$and $Z^{0}$, providing a major triumph of the Standard Model.

The problem remains that spontaneous symmetry breaking predicts the existence of a massive neutral scalar particle referred to as the Higgs boson, which has not been discovered. In addition, its mass depends on a coupling which is unknown, thus the mass cannot be predicted. If it is assumed that the coupling is $\mathcal{O}(1)$, then the Higgs mass will be $\mathcal{O}(100 \mathrm{GeV})$, within the energy regime of current experiments ${ }^{3}$. The search for the elusive Higgs boson is one of the most important reasons for

\footnotetext{
${ }^{3}$ The experimental limit of the Higgs mass is $\mathrm{m}_{H}>114 \mathrm{GeV}$ at $95 \%$ confidence level [18].
} 
building the Large Hadron Collider (LHC).

There are no known deviations from the Standard Model up to energies currently being probed. However, even with its success, it is known not to be a complete theory. This is obvious since the Standard Model does not contain the gravitational interaction. Besides the omission of gravity, too many other questions still remain. Why are there so many free parameters? Why are there three families of quarks and leptons? Why is there such a large matter-antimatter asymmetry in the universe? These types of questions seem to suggest that there are new physics phenomena at energies above the electroweak energy scale.

One serious theoretical problem manifests itself in the separation of scales seen between the electroweak energy scale and the Planck scale, a separation of 17 orders of magnitude. The trouble lies in the fact that the Higgs is a scalar particle with no symmetries preventing it from obtaining as large a mass as possible. Unlike vector bosons, mass terms for scalar particles are gauge invariant. Thus, if new physics does not exist between the electroweak scale and the Planck scale, the Higgs mass would be 17 orders of magnitude larger than the "natural" assumption of $\mathcal{O}\left(10^{2} \mathrm{GeV}\right)$ given above. This is known as the hierarchy problem.

As indicated above, symmetries are usually involved when dealing with the problem of "fine-tuning" physical values, as needed with the mass of the Higgs. The situation may not be different in this case. The introduction of a symmetry between fermions and bosons can explain the relatively low mass of the Higgs boson. This type of symmetry is referred to as supersymmetry and is one of the favoured extensions to the Standard Model. However, a Higgs mass set at the electroweak scale requires supersymmetry to be introduced at energies on or below $\mathcal{O}(1 \mathrm{TeV})$. Again, this is well within the reach of the LHC and will be a major focus of physics studies. 
An experimental motivation for new physics is the observation of cold dark matter (CDM) present in the universe. The Wilkinson Microwave Anisotropy Probe (WMAP) $[19,20]$ determined the difference between the total matter density of the universe and the baryon density, providing a value for the dimensionless quantity representing the cold dark matter energy density:

$$
\Omega_{C D M} h^{2}=0.1126_{-0.0181}^{+0.0161} \quad(2 \sigma-\text { level })
$$

In Equation (1.1), $\Omega_{C D M}$ is the ratio of the cold dark matter density to the critical density and $h /(100 \mathrm{~km} / \mathrm{s} / \mathrm{Mpc})$ is the Hubble constant. Measurements of the dark matter density constrain any models of new physics and although many scenarios exist which attempt to explain dark matter, weakly interacting massive particles with masses and interaction rates characterized by the electroweak scale provide the most compelling alternative. Stable particles with these properties arise naturally in supersymmetric theories, on which more detail will be provided in Section 4.1.

Other models exist for new physics phenomena such as large extra dimensions, quark compositeness, theories with extra gauge bosons, etc.. All theories attempt to answer some of the questions given above, but will not be discussed further here.

Located at the LHC, the ATLAS detector will be utilized in the search for supersymmetry. Both the LHC and ATLAS will be described in Chapter 2 and Appendix A. Calorimetry plays a key role for all physics studies, including searches for new physics phenomena, and will be explained in Chapter 3, along with studies of the ATLAS Forward Calorimeter. Supersymmetry studies using the ATLAS detector will be explored in Chapter 4, Appendix B and Appendix C. A particular particle spectrum in which a light supersymmetric partner to the top quark occurs is presented and the possibility for discovery is investigated. Finally, many projects have been completed over the course of these studies, not all of which are presented 
in this thesis, but a complete list is presented in Appendix D. 


\section{Chapter 2}

\section{The LHC and ATLAS}

\subsection{The Large Hadron Collider}

The Large Hadron Collider (LHC) has been built at the European Organization for Nuclear Research (CERN) in Geneva, Switzerland to collide protons from which precise measurements of Standard Model parameters and searches for new physics phenomena can be made. It is an accelerator ring approximately $27 \mathrm{~km}$ in circumference in which protons will collide with an unprecedented centre-of-mass energy of $\sqrt{s}=14 \mathrm{TeV}$ and a peak luminosity of $L=10^{34} \mathrm{~cm}^{-2} \mathrm{~s}^{-1}$. The properties of the machine are summarized in Table 2.1 and a discussion of the concept of luminosity is given in Appendix A.

The LHC tunnel is built at an average depth of $100 \mathrm{~m}$ underground, straddling the Swiss-French border and is the home to four main experiments. ATLAS (A Toroidal LHC ApparatuS) and CMS (Compact Muon Solenoid) are general purpose experiments that will perform their studies with a variety of physics channels. $\mathrm{LHCb}$ 


\begin{tabular}{l|c} 
Description & Quantity \\
\hline \hline Circumference & $26659 \mathrm{~m}$ \\
Energy (protons) & $7 \mathrm{TeV}$ \\
Design luminosity & $10^{34} \mathrm{~cm}^{-2} \mathrm{~s}^{-1}$ \\
Number of bunches per proton beam & 2808 \\
Number of protons per bunch & $1.15 \times 10^{11}$ \\
Bunch spacing (time) & $25 \mathrm{~ns}$ \\
Number of collisions per second & $\sim 10^{9}$
\end{tabular}

Table 2.1: Design quantities for the LHC.

will be dedicated to the study of $b$-quark physics. In addition to proton-proton collisions, the LHC will also collide heavy ions. In particular, lead nuclei $\left({ }^{208} \mathrm{~Pb}^{+82}\right)$ will collide with energies of $2.76 \mathrm{TeV}$ per nucleon and a luminosity of $10^{27} \mathrm{~cm}^{-2} \mathrm{~s}^{-1}$. With these collisions, ALICE (A Large Ion Collider Experiment) will study the physics of strongly interacting matter at extreme energy densities.

\subsubsection{The Accelerator}

The LHC [21] is the last stage of the CERN Accelerator Complex, illustrated in Figure 2.1, which will accelerate the protons to $7 \mathrm{TeV}$. The protons are initially accelerated to an energy of $50 \mathrm{MeV}$ using the linear accelerator (LINAC) and then injected into the Proton Synchrotron Booster (PSB) where they undergo further acceleration to reach an energy of $1.4 \mathrm{GeV}$. After the PSB, the protons reach an energy of $25 \mathrm{GeV}$ in the Proton Synchrotron (PS). This is followed by the Super Proton Synchrotron (SPS), where they obtain an energy of $450 \mathrm{GeV}$. Finally, the protons are transferred to the LHC where they are accelerated for approximately 20 


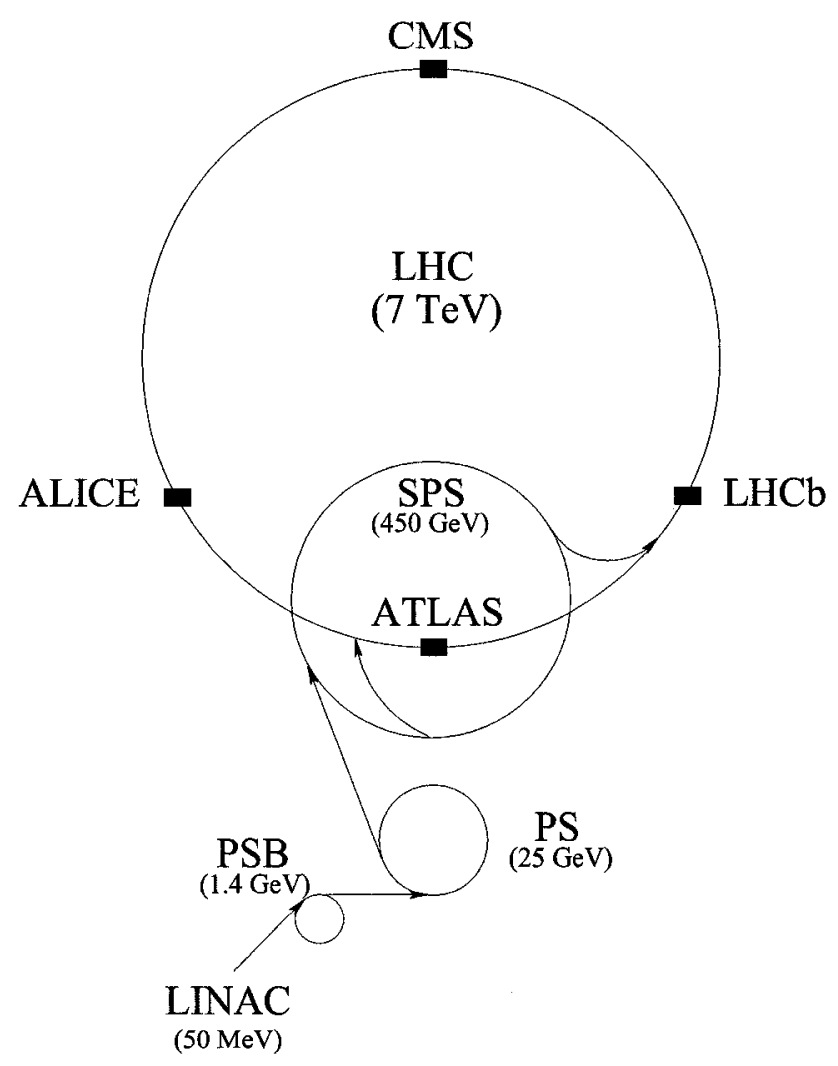

Figure 2.1: Schematic diagram of the CERN Accelerator Complex. Protons are accelerated at different stages until they reach $7 \mathrm{TeV}$ in the LHC.

minutes until they reach the design energy of $7 \mathrm{TeV}$.

The proton energy in the LHC is obtained using superconducting dipole magnets and radio frequency $(\mathrm{RF})$ cavities. The dipole magnets are responsible for bending the beam and defining the beam momentum. Given the bending radius of $2805 \mathrm{~m}$ at the LHC, a proton beam with a momentum of $7 \mathrm{TeV}$ requires a magnetic field of $8.33 \mathrm{~T}$. The RF cavities are electromagnetic resonators responsible for accelerating the protons. The acceleration from $450 \mathrm{GeV}$ to $7 \mathrm{TeV}$ is done over many cycles with each turn producing a gain in energy of approximately $0.5 \mathrm{MeV}$. In addition, quadrupole and higher order magnets are used to focus and stabilize the beams. 
The proton beams at the LHC are composed of well-defined bunches, with each bunch consisting of $1.15 \times 10^{11}$ protons and each beam holding 2808 bunches separated by 25 ns. Because of the short bunch separation, events from more than one bunch crossing may be measured together. This is one source of background known as pileup and the detectors will need to rely on fast readout electronics to avoid sampling events from several different bunch crossings simultaneously.

Employing protons permits high energy collisions because the loss of energy due to synchrotron radiation from the accelerating protons is suppressed by its large mass. It also gives rise to the high luminosity because proton beams are easier to store than anti-proton beams. One drawback to this design is that colliding two counter-rotating proton beams requires the beams to be stored in separate vacuum chambers with opposite magnetic field directions.

\subsubsection{Proton-Proton Interactions}

An important consideration in proton-proton collisions is the fact that the proton is not an elementary (point-like) particle. The proton consists of three valence quarks (two $u$-quarks and a $d$-quark) along with a sea of virtual quarks and gluons. Collectively, these particles are referred to as partons.

In high energy proton-proton collisions, it is the partons that interact. A schematic diagram of proton-proton collisions is given in Figure 2.2. Because each parton only carries a fraction of the total proton momentum, the energy of the interaction will on average, be less than the total available energy. Furthermore, due to the event-by-event variation in the momentum fraction carried by the partons, momentum cannot be constrained in the direction of the beam. The only momentum 


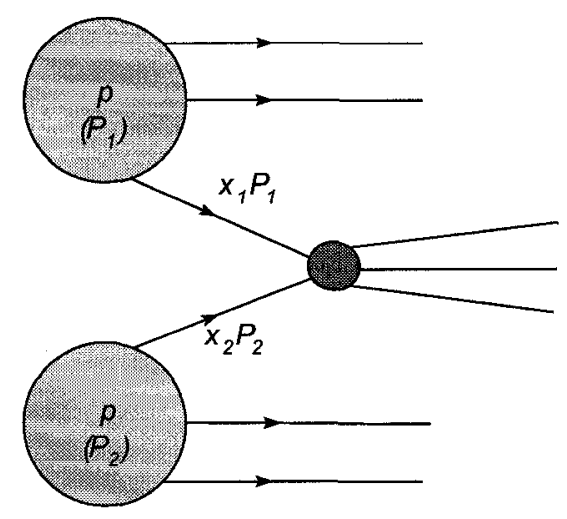

Figure 2.2: Schematic diagram for a proton(p)-proton interaction.

The partons carry an fraction $\mathrm{x}$ of the total proton momentum P.

constraint available is in the plane transverse to the beam axis, referred to as the transverse momentum $\left(p_{T}\right)$. The physics signals of interest consist predominantly of final states with particles having large $p_{T}$.

The cross-section and event rates for proton-proton interactions at peak luminosity are illustrated in Figure 2.3 along with the values for some physics processes of interest at the LHC. The total cross-section $(\sigma)$ is approximately $100 \mathrm{mb}$. This cross-section can be separated into elastic and inelastic scattering events, with $\sigma_{\text {elastic }} \approx 20 \mathrm{mb}$ and $\sigma_{\text {inelastic }} \approx 80 \mathrm{mb}$. Elastic scattering events will not be seen in the detectors because they do not produce particles at sufficient angles relative to the beam line.

The physics events of interest will come from the inelastic events in which the momentum transfer between partons is large, known as hard scattering events. The low event rates for such processes mean that the collisions will be dominated by soft scattering events, producing several events simultaneously. The large cross-section for inelastic scattering produces an average of 25 events per bunch crossing ${ }^{1}$. The

\footnotetext{
${ }^{1}$ This result is for peak luminosity and accounts for gaps in the proton bunches which changes
} 


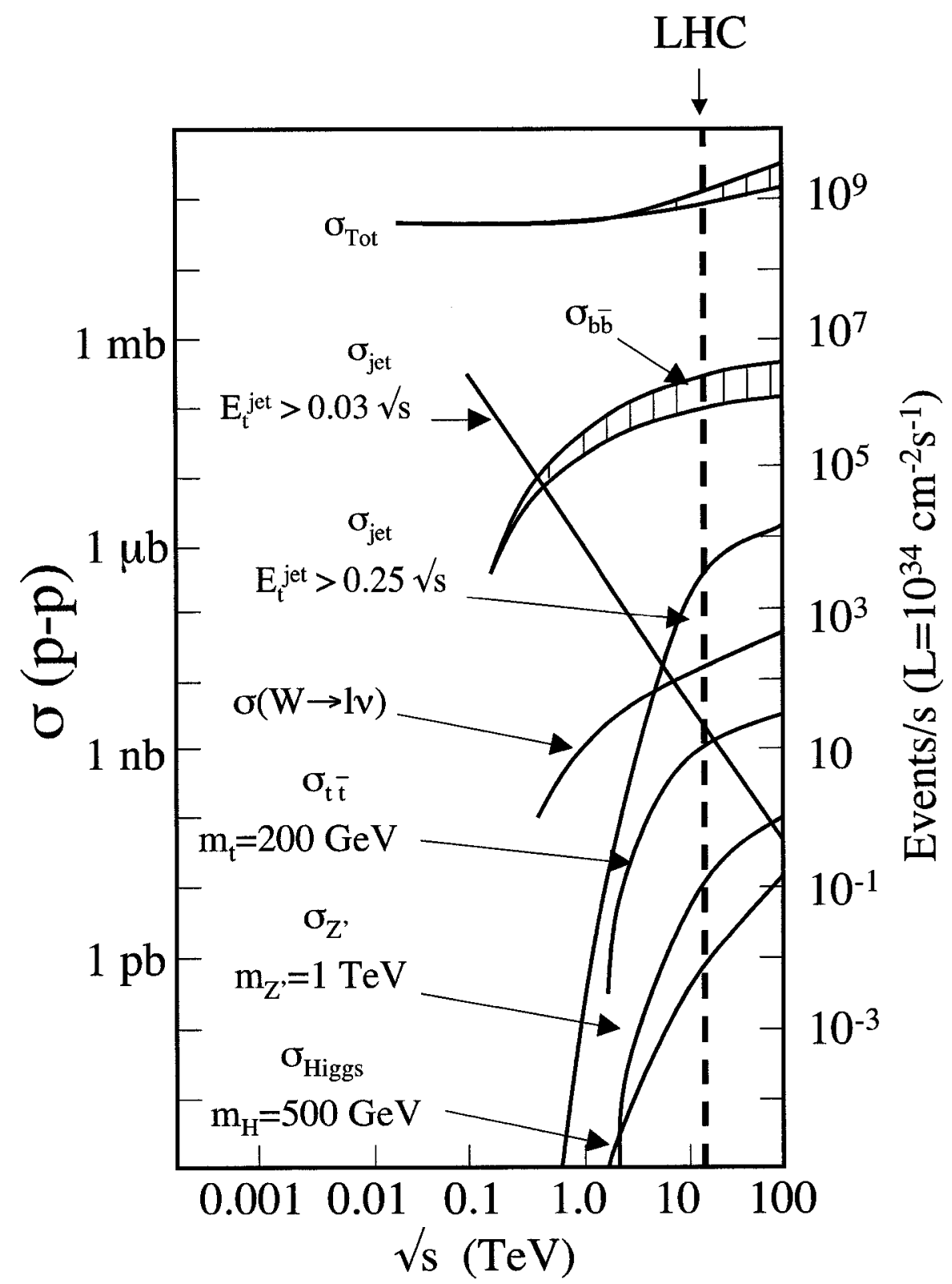

Figure 2.3: Cross-sections for various physics processes in protonproton collisions. 


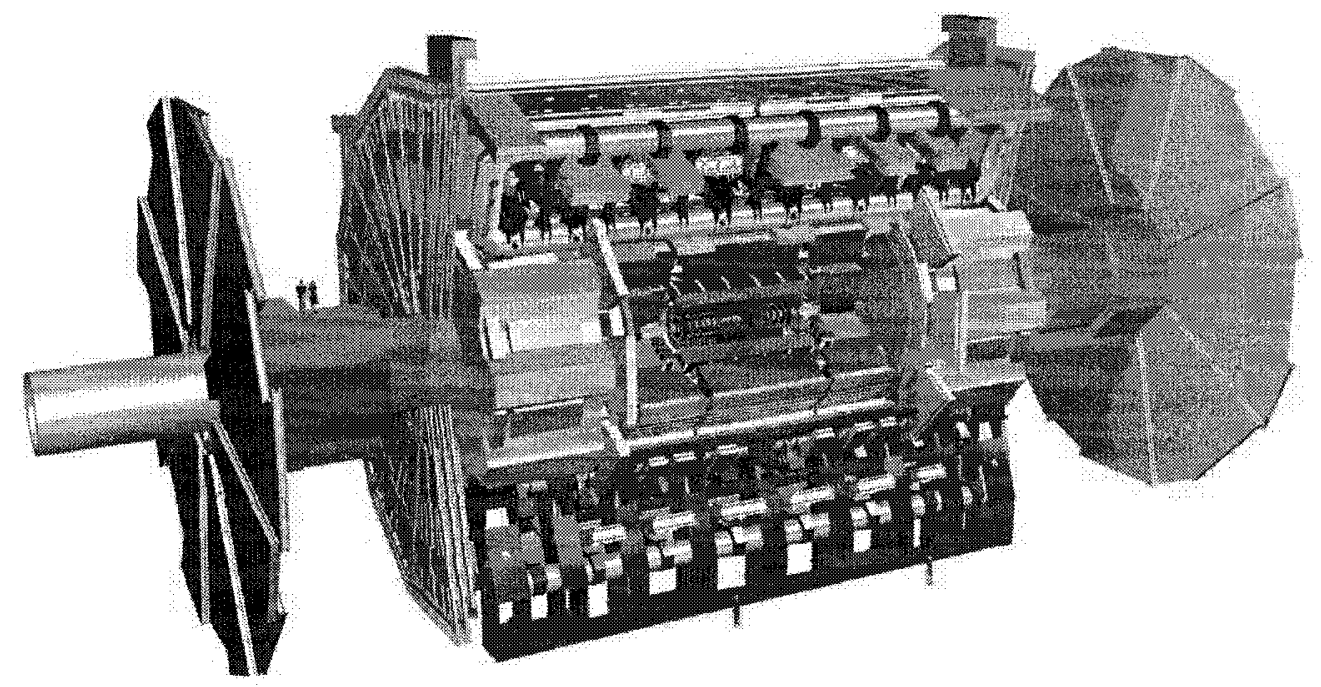

Figure 2.4: Schematic diagram of the ATLAS detector and its subsystems.

large number of events per bunch at peak luminosity adds to the pileup mentioned above and will be an important source of background for all physics studies.

\subsection{The ATLAS Experiment}

The ATLAS collaboration consists of approximately 2500 people and has defined a very extensive physics program. Studies will be performed to understand the nature of electroweak symmetry breaking, to reduce uncertainties on Standard Model parameters and to search for new physics phenomena. The ATLAS detector [22], shown in Figure 2.4, has been built to fulfill the requirements for the study of most of the physics that may appear at the $\mathrm{TeV}$ scale.

As described in Section 1.1, one possible solution to electroweak symmetry breaking is the Higgs mechanism. The discovery of the Higgs boson was used as a benchmark analysis in the design of the ATLAS detector. It underlies the robustthe effective bunch separation from $25 \mathrm{~ns}$ to $31.6 \mathrm{~ns}$. 


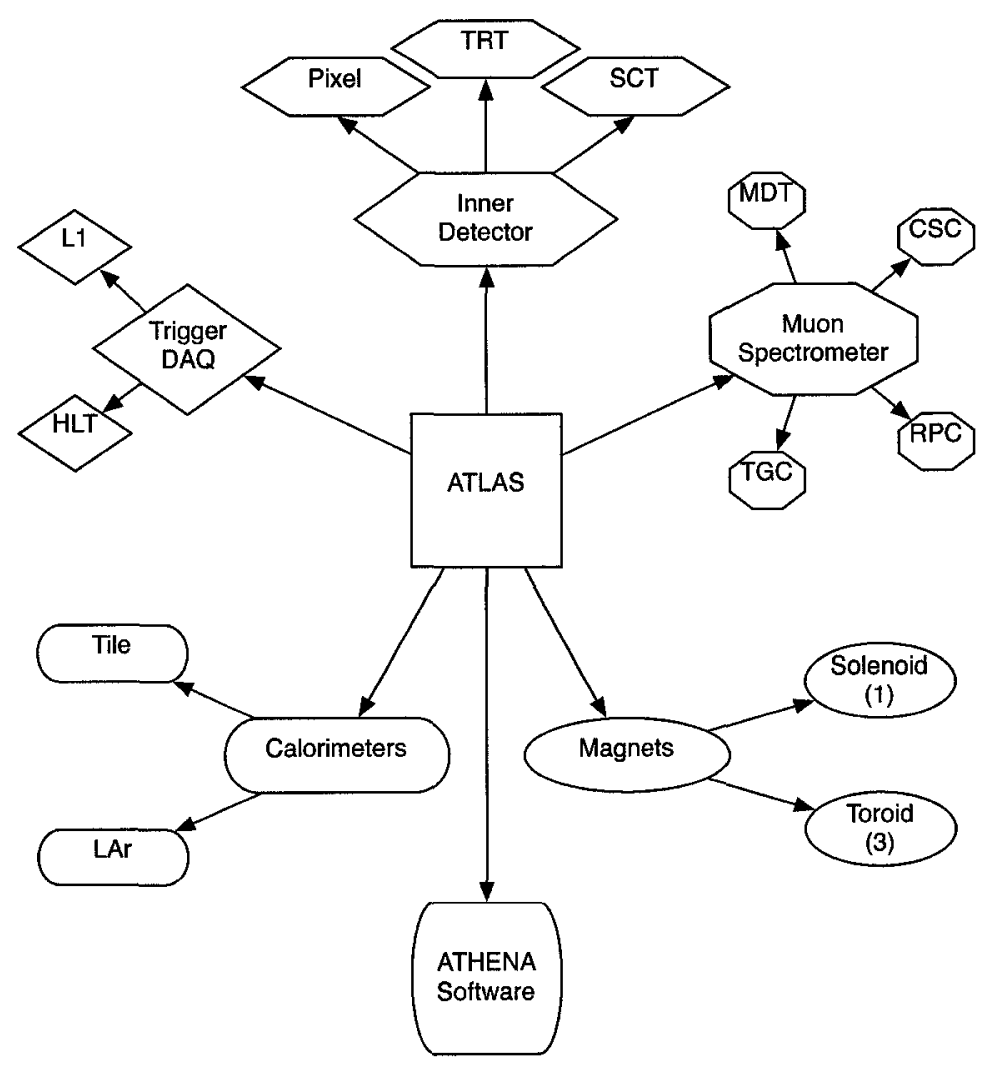

Figure 2.5: List of the ATLAS detector components and their subsystems.

ness of the detector because a range of production and decay mechanisms can occur depending on the mass of the Higgs, leading to a variety of possible signatures.

The high luminosity of the LHC will allow for precise tests of QCD and measurements of electroweak interactions. Also, the top quark will be produced in abundance, allowing for more precise measurements of its properties, including the top mass and the cross-section for single-top production.

Theories beyond the Standard Model which are accessible with the ATLAS detector are supersymmetry, quark compositeness, theories with extra gauge bosons as well as theories with extra dimensions. A common signature to many theories extending the Standard Model is large amounts of energy which go undetected, referred 
to as missing energy $(\mathbb{E})$.

The wide range of physics studies requires certain characteristics of the ATLAS detector. Full coverage around the beam axis is important for minimizing backgrounds for missing energy. Particle identification techniques for electrons, photons and muons as well as hadronic calorimetry are also essential for jet and missing energy measurements.

In order to fulfill the requirements of the physics goals, the ATLAS detector is divided into a number of subdetectors which have specific characteristics. A list of the different components and their subsystems is given in Figure 2.5. The major hardware components of the ATLAS detector are the magnet system, the inner detector, the calorimeters, the muon chambers and the trigger/data acquistion system. In addition, the software system, referred to as ATHENA, is an integral part of the ATLAS experiment. Although the systems are represented as independent subsystems, many work together to perform measurements. For example, the magnets are essential components and together with the muon chambers form the muon spectrometer, which identifies muons and measures their momentum. The subsystems will be described in more detail in the upcoming sections.

The ATLAS detector is a large cylindrical detector with a length of $44 \mathrm{~m}$ and a diameter of $25 \mathrm{~m}$. The coordinate system is defined such that the $z$-axis is aligned with the beam axis. The $x y$-plane is transverse to the beam axis with the $+x$ pointing towards the center of the LHC ring and the $+y$-axis pointing upward. The azimuthal angle $\phi$ is measured around the beam axis and the polar angle $\theta$ is measured from the $z$-axis. The transverse momentum $\left(p_{T}\right)$ and transverse energy (defined as $E_{T}=E \sin \theta$ ) are defined in the $x y$-plane. A common variable that is used in the description of the detector is referred to as the pseudo-rapidity, $\eta$. It is 
defined as

$$
\eta \equiv-\ln \left(\tan \left(\frac{\theta}{2}\right)\right)
$$

\subsubsection{Inner Detector}

The ATLAS inner detector (ID) $[23,24]$ is designed to reconstruct and measure the trajectory and momentum of charged particles, as well as locating primary and secondary vertices in physics events. Three separate but complementary subdetectors perform the measurements. The silicon pixel and silicon microstrip (SCT) detectors provide high resolution measurements at the inner radii of ATLAS, while the transition radiation tracker (TRT) provides continuous tracking at the outer radii. All three subdetectors of the inner detector are immersed in a $2 \mathrm{~T}$ magnetic field generated by the central solenoid.

The inner detector is illustrated in Figure 2.6. It has components in both the barrel region and the end-cap regions, providing coverage over $|\eta|<2.5$ for momentum measurements and $|\eta|<2.0$ for electron identification. The pixel detector consists of three cylinders concentric to the beam axis and three disks perpendicular to the beam axis in each of the end-caps, while the SCT has 4 layers in the barrel and 9 disks in each of the end-caps. The TRT consists of 4-mm-diameter polyimide straws arranged parallel to the beam axis in the barrel region and radially in the endcap region, extending the tracking measurements to $|\eta|=2.0$. The 80.4(6.3) million readout channels in the pixel detector (SCT) provide the high granularity needed for the excellent spatial resolution [22]. With an average of 36 hits per track, the TRT provides continuous track measurements to improve the momentum resolution and particle identification capabilities of the inner detector. 


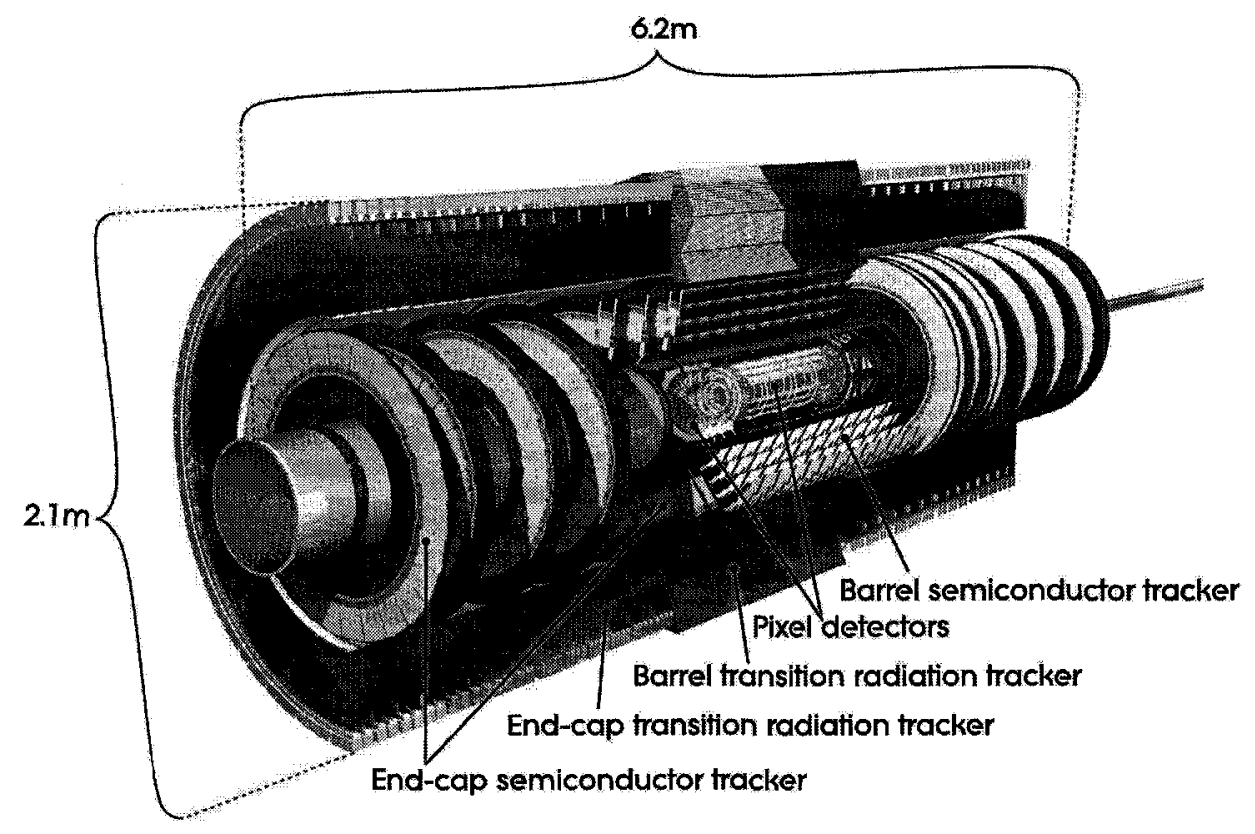

Figure 2.6: The ATLAS inner detector.

\subsubsection{Calorimeters}

The ATLAS calorimeter system [25,26], illustrated in Figure 2.7, contains many different subdetectors to measure the energies of electrons, photons and jets. Both electromagnetic (EM) and hadronic (H) calorimeters are located in the barrel (B) and end-cap (EC) regions, providing coverage over $|\eta|<4.9$. The individual detectors consist of the electromagnetic barrel (EMB), the Tile barrel, the Tile extended barrel, the electromagnetic end-cap (EMEC), the hadronic end-cap (HEC) and the forward calorimeter (FCal). Not labelled in the figure is a presampler detector covering the region $0<|\eta|<1.8$, used to correct for the energy lost by electrons and photons upstream of the calorimeter. The EM calorimeters are 22 (24) radiation lengths, while the hadronic calorimeters are 9.7 (10) interaction lengths in the barrel (endcap) region. The amount of material sufficiently absorbs highly energetic particles, satisfying energy resolution requirements as well as shielding for the muon system. 


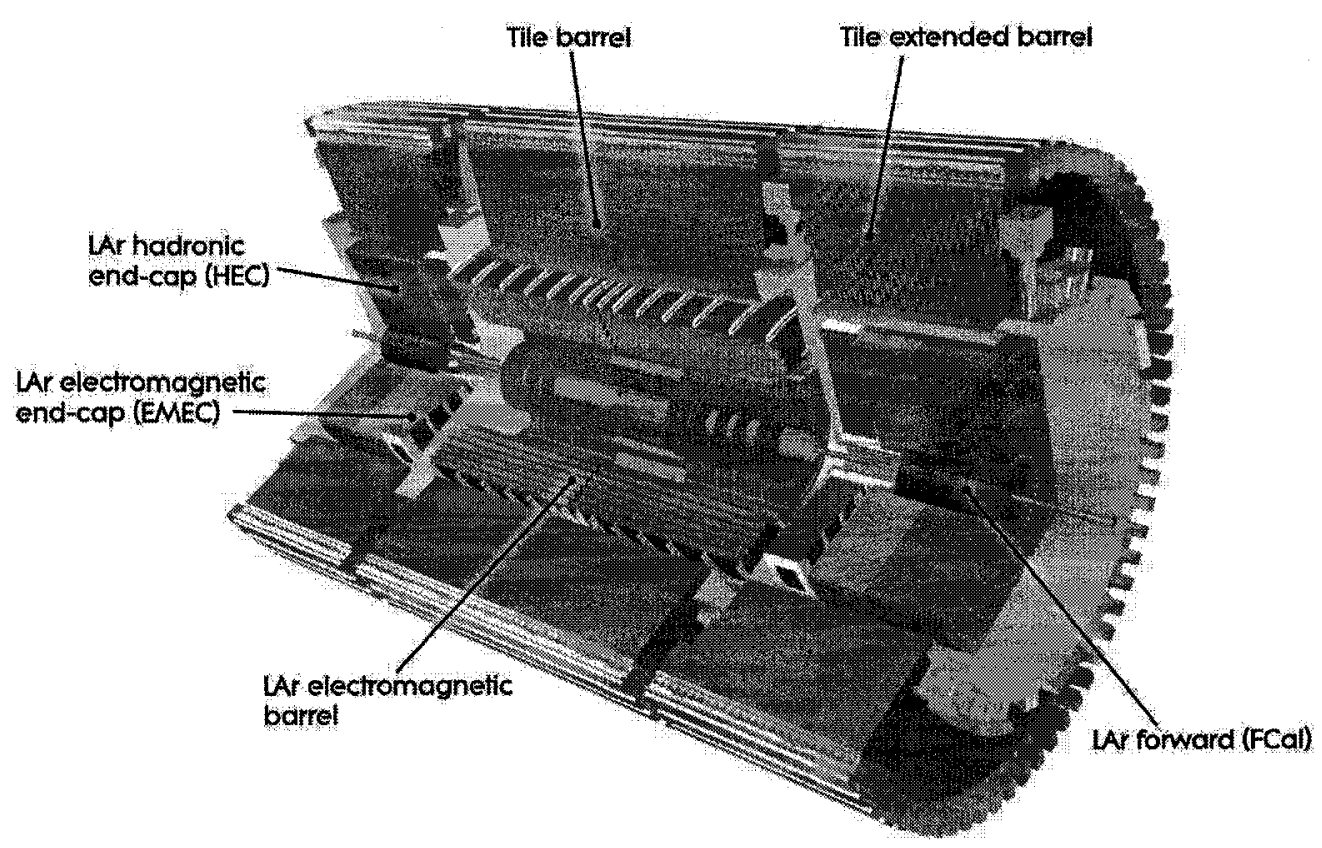

Figure 2.7: The ATLAS calorimeter system.

The fine granularity of the EM readout system in the region overlapping the ID allows for precise measurements for electrons and photons, while the more coarse granularity in the other calorimeters is sufficient for jet and ${ }_{T}$ measurements [22].

All of the calorimeters in the ATLAS detector are sampling calorimeters. Liquid argon (LAr) is the active medium in the EM barrel, EMEC, HEC and FCal, while scintillating tiles are used in the Tile calorimeters. Liquid argon was chosen for its radiation hardness as well as its relatively low cost [27]. More information about sampling calorimeters can be found in Section 3.1.3.

The EMB covers the region $|\eta|<1.475$, while the EMEC covers the region $1.375<|\eta|<3.2$. Both systems use lead as the absorbing material, arranged in an accordion geometry to provide complete symmetry in $\phi$ with no azimuthal cracks. The accordion geometry is shown in Figure 2.8a. 


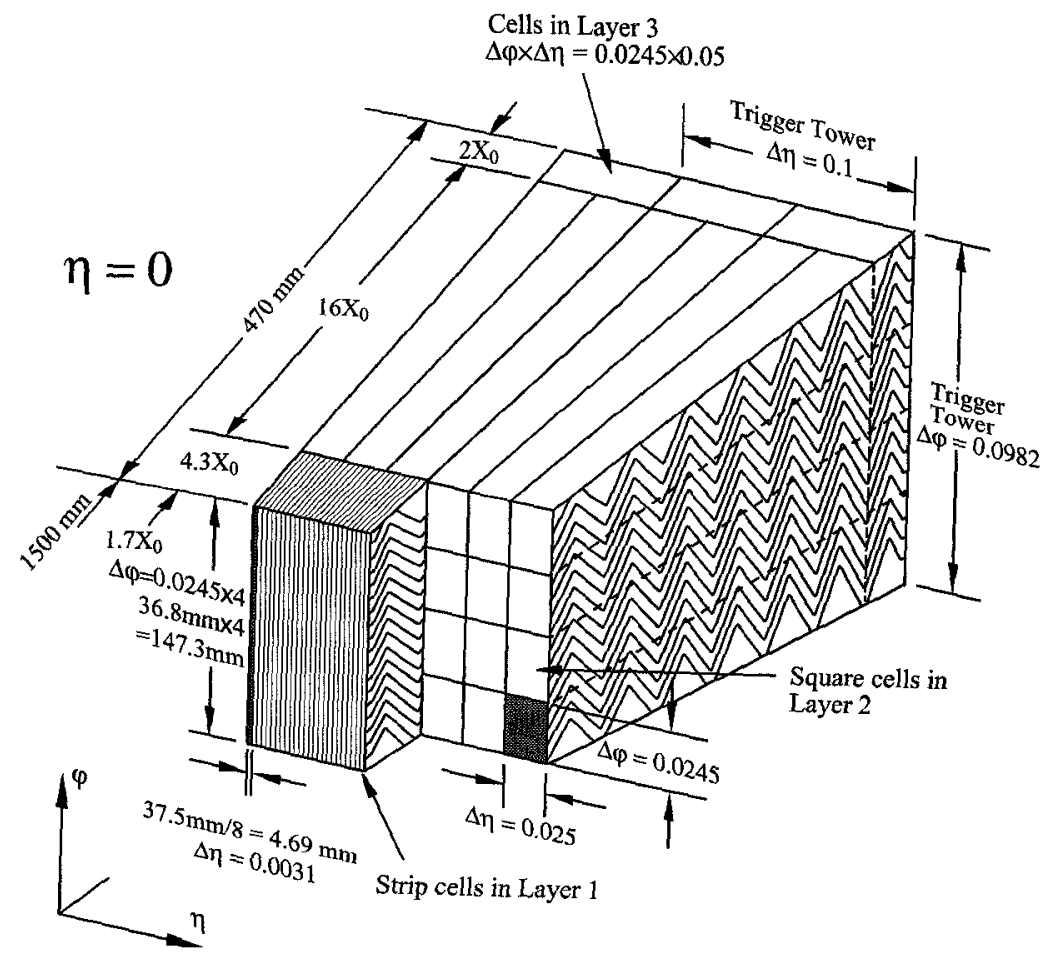

(a) Electromagnetic barrel calorimeter.

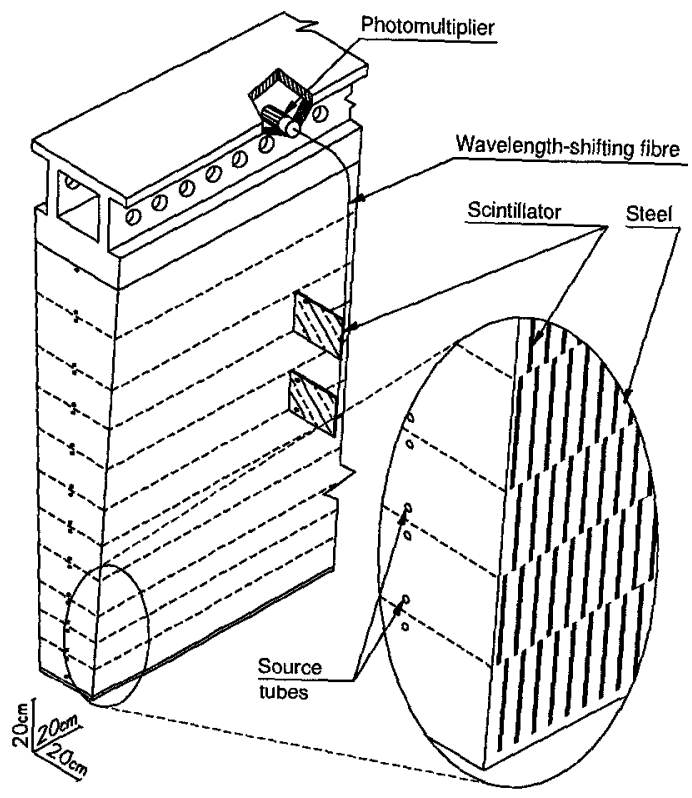

(b) Tile barrel calorimeter.

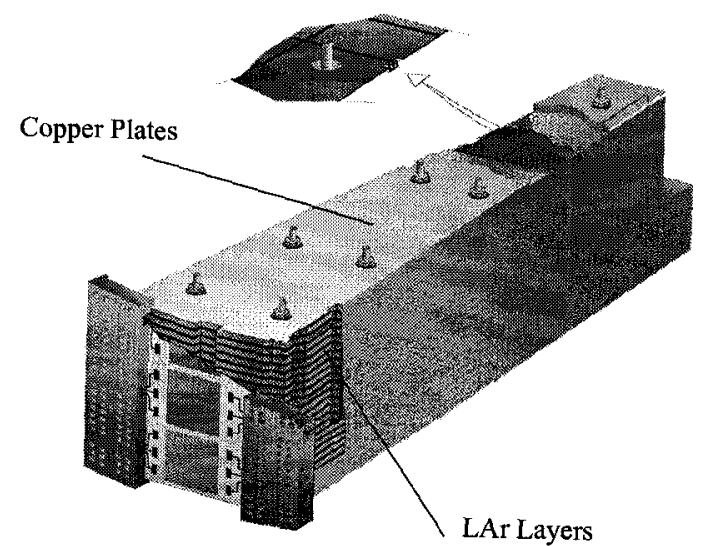

(c) HEC module.

Figure 2.8: Diagrams for sections of the ATLAS calorimeters. 
The Tile barrel detector provides hadronic calorimetry for the region $|\eta|<1.0$ while the Tile extended barrel detector expands the coverage to include $0.8<|\eta|<1.7$. One section of the Tile barrel is illustrated in Figure 2.8b. Steel plates are used as the absorbing material and $3 \mathrm{~mm}$ thick plastic scintillating tiles are used as the active material.

The hadronic end-cap calorimeter is a copper-LAr sampling calorimeter located in each of the end-cap cryostats, placed just behind the EMEC. The HEC consists of two independent wheels, built from 32 identical wedge-shaped modules and covers the region $1.5<|\eta|<3.2$. One HEC module is illustrated in Figure 2.8c.

The forward calorimeter completes the hermetic design of the ATLAS calorimeter system, located within the same cryostat as the EMEC and HEC. Each FCal consists of three modules with the module closest to the interaction point optimized for electromagnetic shower measurements and the other two for hadronic shower measurements. Each module has similar designs, with a metal absorber matrix and cylindrical electrodes oriented parallel to the beam axis. The electrode structure is a novel design, consisting of copper tubes and metal rods forming concentric cylinders. The metal rods are constructed from the same material as the absorbing matrix; copper in the case of the EM module and tungsten in the hadronic modules. More details about the FCal will be provided in Chapter 3 .

\subsubsection{Muon Spectrometer}

The muon spectrometer [28] surrounds the calorimeter system and defines the spatial extent of the ATLAS detector. A layout of the spectrometer is provided in Figure 2.9. It is designed to detect charged particles which pass beyond the barrel and end-cap 


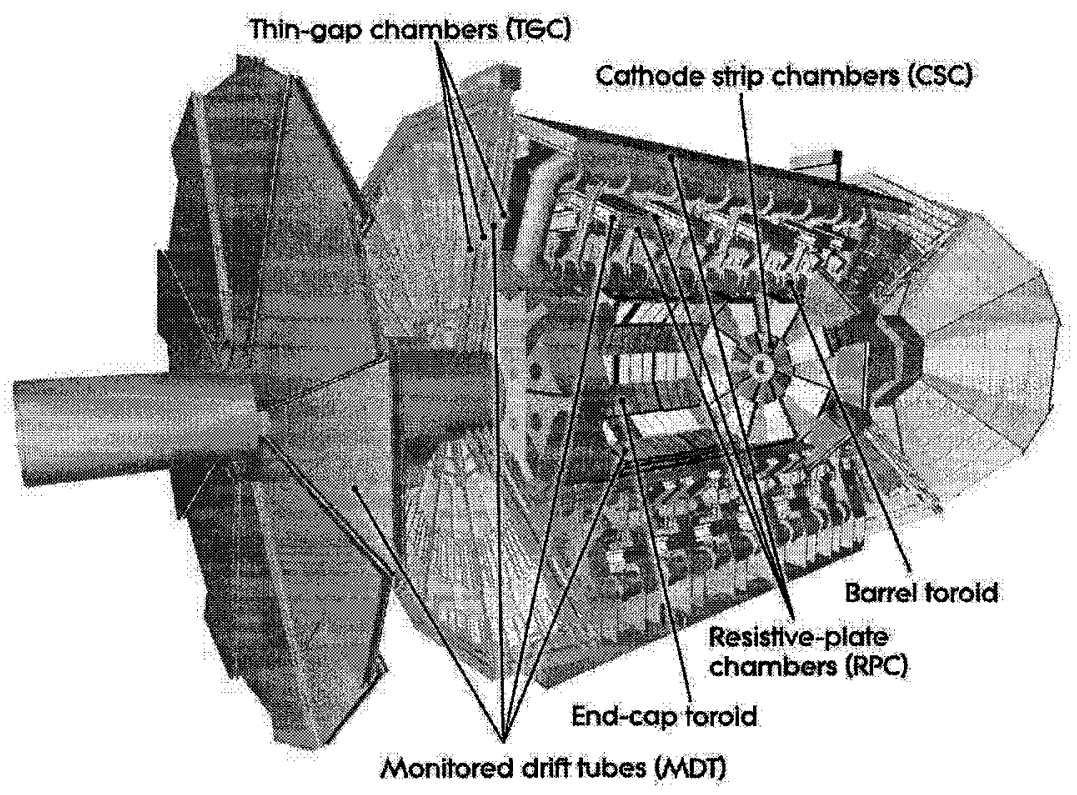

Figure 2.9: Cut-away view of the muon spectrometer.

calorimeters and measure their momentum. The momentum measurements are based on the deflection of the charged particles in magnetic fields generated by three large superconducting air-core toroid magnets (one barrel and two end-caps). The muon system is capable of precise momentum measurements in the region $|\eta|<2.7$. For the region $|\eta|<2.7$, the precision momentum measurements are performed by the Monitored drift tube chambers (MDT's), while the Cathode strip chambers (CSC's) are used in the first layer of the region $2.0<|\eta|<2.7$. The precision-tracking chambers in the barrel region are located on and between the barrel toroid magnets, forming concentric cylindrical shells around the beam axis at radii of $5 \mathrm{~m}, 7.5 \mathrm{~m}$ and $10 \mathrm{~m}$. In the two end-caps, the chambers form large wheels perpendicular to the $z$-axis, placed in front and behind the end-cap toroids at $7.4 \mathrm{~m}, 10.8 \mathrm{~m}, 14 \mathrm{~m}$ and $21.5 \mathrm{~m}$ from the interaction point.

The ability to trigger on muons, essential in the design of the muon spectrometer, is accomplished using the Resistive-plate chambers (RPC's) in the barrel 
region and the Thin-gap chambers (TPG's) in the end-cap region. The muon trigger system provides track information within a few tens of nanoseconds after passage of a particle. The muon trigger system provides well defined $p_{T}$ thresholds, provides bunch-crossing identification and measures the muon's coordinates in the direction perpendicular to that determined by the precision-tracking chambers.

\subsubsection{Trigger}

The trigger system $[29,30]$ is responsible for reducing the large event rate $(1 \mathrm{GHz})$ in the p-p collisions at the LHC, keeping only events with signatures defined by the different physics analysis groups. The ATLAS trigger system consists of the level-1 (L1) and level-2 (L2) triggers and the event filter. Together, the level-2 trigger and the event filter form the high level trigger. Each level in the trigger system refines the decisions made in the previous step and also provides additional selection criteria wherever possible. The design goal of the trigger system is to reduce the event rate to approximately $200 \mathrm{~Hz}$.

The L1 trigger is the first step in selecting interesting events, reducing the event rate to $75 \mathrm{kHz}$. Initial searches are for high $p_{T}$ particles as well as large $\mathbb{F}_{T}$ and total $E_{T}$. The selection of events is based on information from a subset of detectors. As mentioned in Section 2.2.3, muons are triggered in the barrel and end-cap regions using the RPC's and the TGC's, respectively. The calorimeters are also used, however, at a reduced granularity. Trigger 'menus', which consist of combinations of selection criteria, are implemented for level-1 processing. The trigger menus are defined by the different physics analysis groups who define the relevant signals.

The level-1 trigger also defines Regions of Interest (RoI's). The RoI are regions 
within the detector, defined by $\eta$ and $\phi$, where the selection process has identified certain features; for example, a cluster of energy passing a certain energy threshold. The RoI are used to seed the selections for the high level trigger.

The input to the level-2 trigger is the RoI defined in L1. The L2 trigger employs the full granularity and precision of the detector within the RoI. The trigger menus at this level are designed to reduce the event rate to $3.5 \mathrm{kHz}$.

The final step in the trigger system is the event filter, which reduces the event rate to $200 \mathrm{~Hz}$. This is done using offline analysis procedures with an average processing time of approximately 4 s per event.

\subsubsection{ATHENA Software}

The large scale and complexity of the ATLAS experiment requires the software to be highly modular, robust and flexible enough to meet the needs of the project throughout its lifetime. This is accomplished through the design of the ATHENA software framework [31].

The goal is to implement software capabilities for all stages of the experiment in one common framework; physics and detector simulation, reconstruction of physics objects, visualization, analysis tools, etc., are all embedded into ATHENA. This approach assures developers and users that the same software tools will be available for various applications.

The ATHENA framework is based on an Object-Oriented design, relying primarily on the $\mathrm{C}++$ and Python languages. The modularity and flexibility follow from the component-based model adopted for the framework. In the case of ATHENA, applications are built up from components (usually written as C++ classes), using 


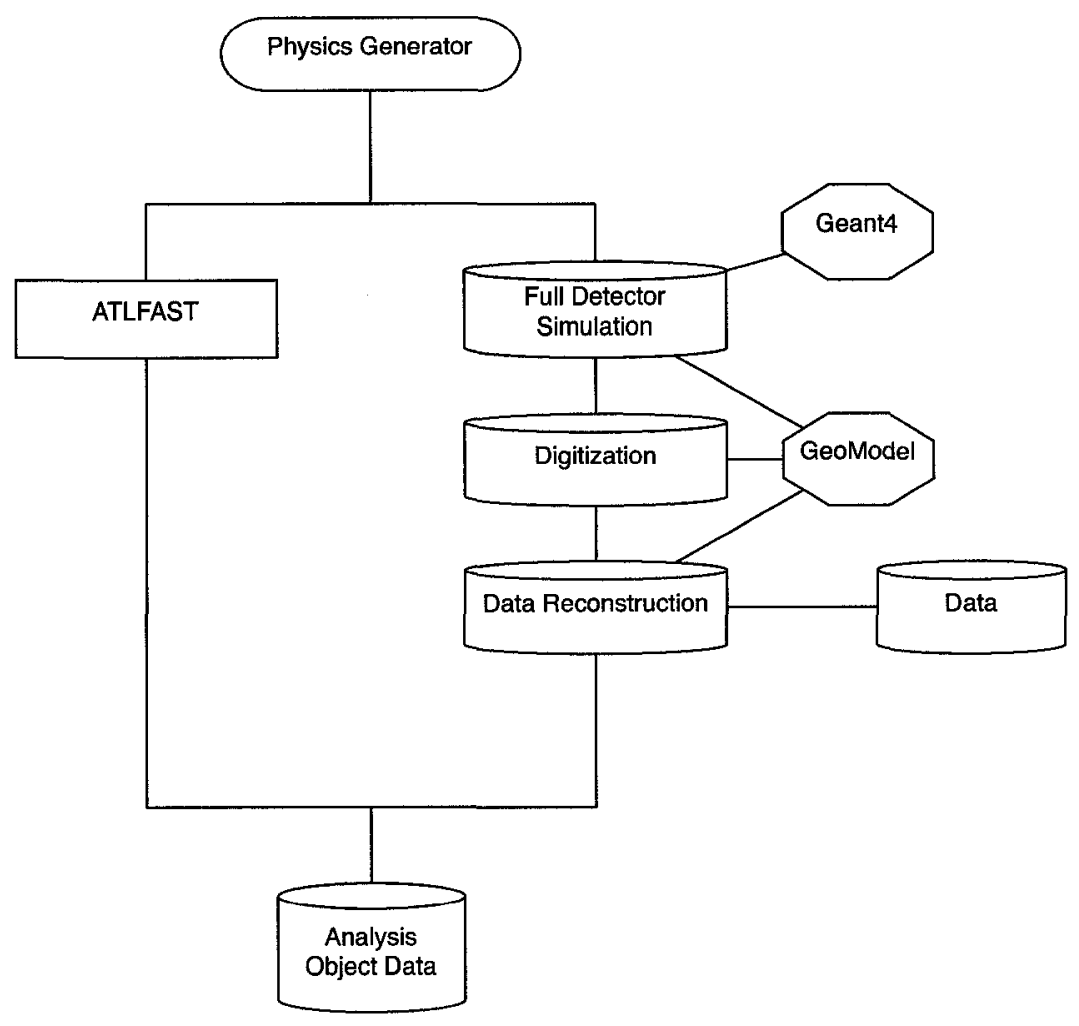

Figure 2.10: Analysis Software Chain.

Python-written configurable files (called jobOptions files).

Figure 2.10 illustrates some components of the software chain. Event generators simulate proton-proton interactions of the LHC, which are used as input for the detector simulation and reconstruction algorithms. The software is designed such that at the reconstruction level, the Monte Carlo and experimental data have identical formats. The same algorithms are used in both cases for reconstructing physics objects such as particle energies, particle identification, etc., and are processed and stored as "analysis object data" for further use in analyses.

Three software components that have been incorporated into ATHENA which are of importance to the work presented here are GeoModel [32], Geant4 [33] and ATLFAST [34]. All three are shown in Figure 2.10. GeoModel and Geant4 were 
essential in the studies of the forward calorimeter and ATLFAST was used in the studies of supersymmetry.

GeoModel is a geometry toolkit designed specifically for use in large, complex particle detectors. The software provides consistent detector geometry description for both the simulation (including digitization) and reconstruction programs, while minimizing computer memory consumption. GeoModel includes mechanisms for obtaining information about the raw geometry of the detector as well as specific subsystem geometrical services, such as the readout geometry.

The simulation of particles interacting with the ATLAS detector is performed by Geant4. The software includes an abundant set of physics models for both electromagnetic and hadronic processes, describing particle interactions with matter over a wide range of energies; from $250 \mathrm{eV}$ in some interactions and extending to the $\mathrm{TeV}$ energy range in others. The user has the option of including individual physics processes for their needs. For example, to study the effect of ionization due to electrons, this process must be included in the user's program. Furthermore, alternate physics models are available for the description of many physics processes. For convenience, Geant4 offers physics lists which combine processes for electromagnetic and hadronic interactions, combining many of the different physics models. The availability of different physics lists is utilized by the ATLAS collaboration and will be discussed further in Chapter 3. GeoModel is responsible for the detector description needed in Geant4 and also provides the tools necessary for the conversion between the two software packages.

ATLFAST provides a parameterized description of the performance of the ATLAS detector and therefore is referred to as a 'fast simulation'. The parameterization is accomplished by smearing the information obtained from the event generator 
with resolution functions which were obtained in studies using the full detector simulation. ATLFAST is generally 4-5 times faster than full simulation and is usually the first step in a physics analysis, performed to determine whether a particular study is feasible with the detector. More information on ATLFAST can be found in Appendix B. 


\section{Chapter 3}

\section{The ATLAS Forward Calorimeter}

Over the past 15 years, many people have been involved with the ATLAS Forward Calorimeter (FCal). From the design of the FCal to the installation and commissioning, the FCal group has evolved, with each member contributing to the understanding of the detector. In this chapter, my contribution, through Monte Carlo simulations of the detector, will be explained. An introduction to calorimetery will be provided, along with details of the forward calorimeter. Finally, results of my Monte Carlo studies will be presented and compared to results from the 2003 beam test data.

\subsection{Calorimetry}

Calorimeters are used to measure the energy of particles by completely absorbing them and converting the deposited energy into a useable signal based on the collection of electric charge or light. Calorimeters play an important role in the ATLAS detector and are designed to measure the energy of electrons, photons and particle jets. 
Calorimeters are divided into two main types of detectors; calorimeters which continuously measure the energy of the particle as it traverses the detector material and calorimeters which periodically sample the energy deposited by the particle [35]. The former are called homogeneous calorimeters and they have the property that the absorbing material and signal generating (active) material are the same. The latter type utilize different materials for the absorbing and active medium and are referred to as sampling calorimeters. Sampling calorimeters will be the focus of this section since all of the ATLAS calorimeters are of this type.

Calorimeters are further categorized as electromagnetic (EM) and hadronic calorimeters. The ATLAS detector contains both EM and hadronic sampling calorimeters, designed to exploit the properties of the different interactions which occur between particles with matter.

\subsubsection{Electromagnetic Showers}

For very energetic electrons and photons which interact with matter via the electromagnetic interaction, the dominant source of energy loss is due to radiative processes [6]. The processes contributing to the energy loss of photons and electrons are shown in Figure 3.1 and Figure 3.2, respectively. As illustrated in Figure 3.1 for photons, the cross-section for the production of electron-positron pairs $(\kappa)$ dominates for highly energetic $(E>100 \mathrm{MeV})$ photons in lead while Figure 3.2 shows that the primary means of energy loss for energetic electrons $(E>10 \mathrm{MeV})$ is by the production of photons through bremsstrahlung. At lower energies, the photoelectric effect $\left(\sigma_{\text {p.e. }}\right)$ dominates photon interactions and ionization dominates energy loss for electrons.

When a highly energetic electron or photon is incident on a thick material, a 


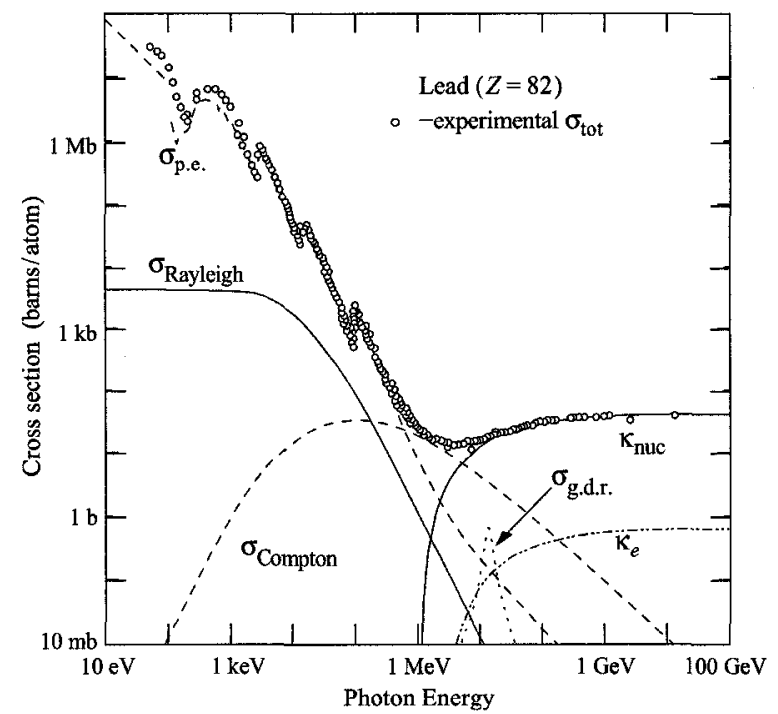

$\sigma_{\text {p.e }}=$ atomic photoelectric effect
$\sigma_{\text {Rayleigh }}=$ Rayleigh scattering
$\sigma_{\text {Compton }}=$ Compton scattering
$\sigma_{\text {g.d.r }}=$ photonuclear interactions
$\sigma_{\kappa_{e}}=$ pair-production (electron field)
$\sigma_{\kappa_{\text {nuc }}}=$ pair-production (nuclear field)

Figure 3.1: Photon cross-sections in lead. At high energies, energy loss due to pair production $(\kappa)$ dominates. Figure taken from [6].

cascade or shower of secondary particles is created through the radiative processes described above. A simplified example of an electromagnetic shower is given in Figure 3.3a for an incident electron. The number of secondary particles increases with depth, but the average energy of the secondaries decreases. The shower continues until the secondaries reach the energy in which ionization dominates the energy loss. The energy at which this occurs is referred to as the critical energy $\left(E_{c}\right)$. Below this energy threshold, not enough energy is available to create new particles and the shower ceases. The critical energy for liquids and solids can be estimated by [36]

$$
E_{c}=\frac{610}{Z+1.24} \quad[\mathrm{MeV}]
$$

where $Z$ is the atomic number of the material. For example, the critical energies for copper and lead are $20.2 \mathrm{MeV}$ and $7.3 \mathrm{MeV}$, respectively.

Two quantities are useful when characterizing the extent of electromagnetic showers. The radiation length $\left(X_{0}\right)$ characterizes the length scale of the longitudinal 


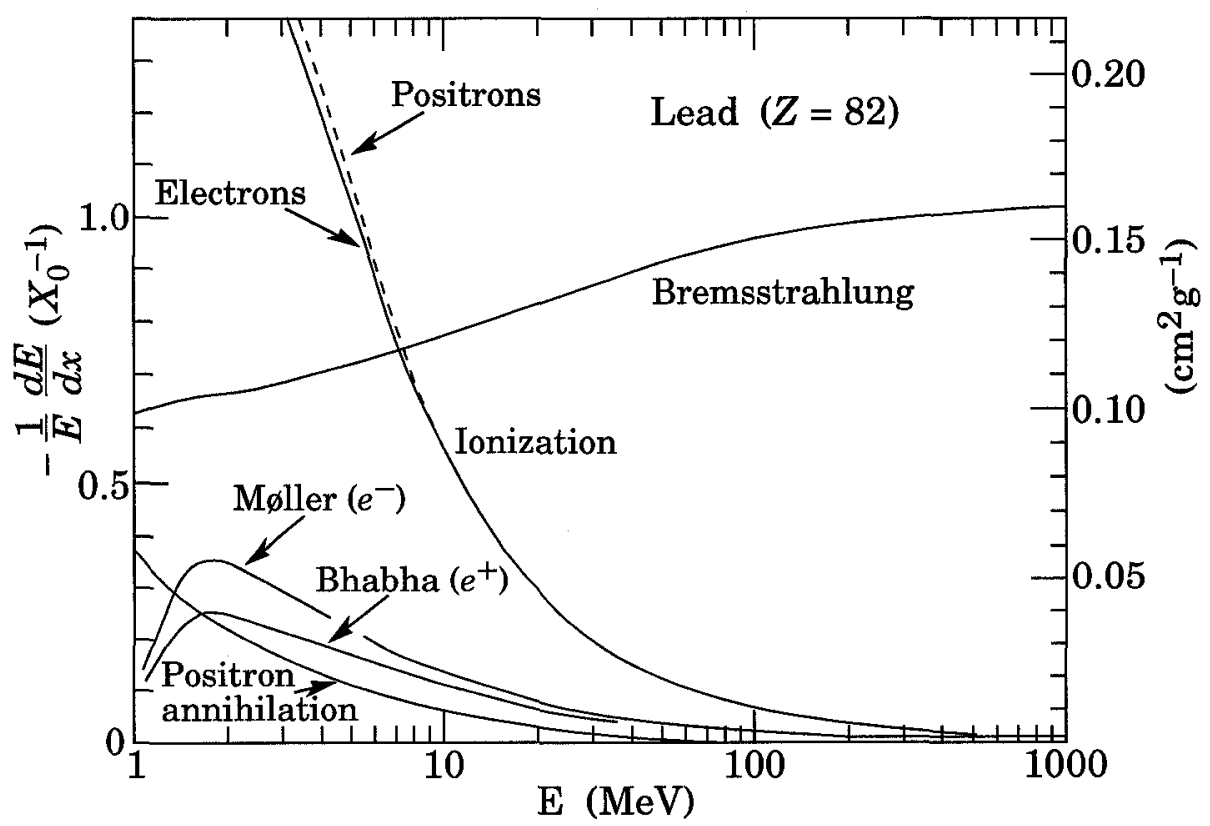

Figure 3.2: Energy loss of electrons in matter. At high energies, energy loss due to bremsstrahlung dominates. Figure taken from [6].

development of the shower while the Molière radius $\left(R_{M}\right)$ characterizes the transverse development of the shower.

The radiation length is defined as the distance over which the energy of the electron is reduced by a factor of $1 / e$, due only to radiation losses. The radiation length for a material can be calculated using [6]

$$
X_{0}=\frac{716.4 A}{Z(Z+1) \ln (287 / \sqrt{Z})} \frac{1}{\rho} \quad[\mathrm{cm}],
$$

where $A$ is the atomic weight of the material and $\rho$ its density, with units of $\left[\mathrm{g} / \mathrm{cm}^{3}\right]$. The radiation length for copper and lead are $1.47 \mathrm{~cm}$ and $0.56 \mathrm{~cm}$, respectively.

The amount of absorber material needed to contain an electromagnetic shower is often quoted in terms of the radiation length. For example, to contain $99 \%$ of the energy of a $100 \mathrm{GeV}$ electron, approximately $17 X_{0}$ of copper is needed [36]. Differ- 
ences in the amount of material required arise because the production of $e^{+} e^{-}$pairs by photons and the emission of bremsstrahlung by charged particles extends down to lower energies in materials with larger $\mathrm{Z}$ values.

The average lateral deflection of electrons with critical energy after traversal of $1 X_{0}$ is given by the Molière radius. Different factors can contribute to the lateral spread of an electromagnetic shower. Electrons and positrons can move away from the shower axis while undergoing multiple scattering. Photons created in bremsstrahlung can travel at angles with respect to the shower axis. Finally, electrons and photons can be created isotropically in processes such as Compton scattering and the photoelectric effect. The Molière radius is parameterized by

$$
R_{M}=\frac{21[\mathrm{MeV}]}{E_{c}} \frac{X_{0}}{\rho} \quad[\mathrm{cm}]
$$

leading to a value of $1.53 \mathrm{~cm}$ for copper and $1.60 \mathrm{~cm}$ for lead. A cylinder of $1 R_{M}$ contains roughly $90 \%$ of the lateral shower in an electromagnetic cascade.

\subsubsection{Hadronic Showers}

When a highly energetic hadron is incident on a thick piece of material, a situation arises analogous to EM showers described above. In the case of hadrons, however, the strong force dominates the interactions instead of the electromagnetic force. A schematic diagram of a hadronic shower is given in Figure 3.3b.

As in the case of EM showers, the interaction of the incoming hadron with the absorber nuclei leads to multi-particle production. Unlike EM showers, however, a simple description of the cascade is not available. The reason for this is that there are in excess of 300 different processes that contribute to the total cross-section, all with approximately the same probability for interaction $(\approx 0.1 \%)$ and not one of them 


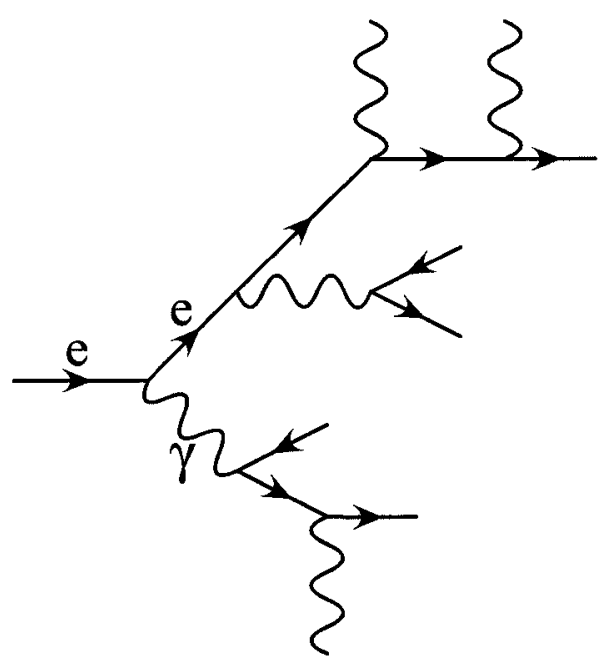

(a) Electromagnetic shower

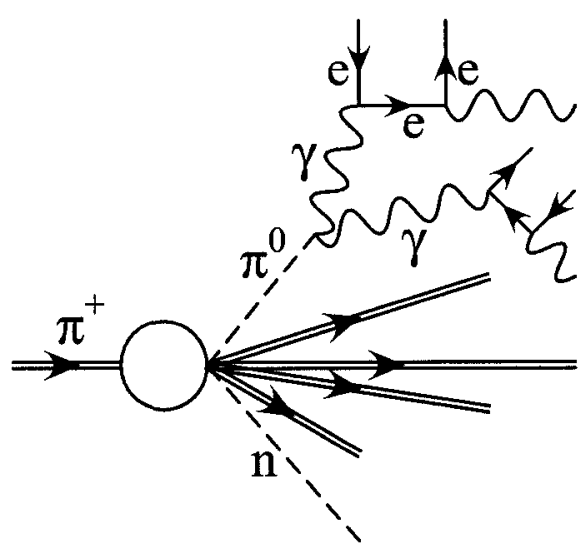

(b) Hadronic shower

Figure 3.3: Schematic illustration of an (a) electromagnetic shower and (b) hadronic shower.

contributing more than $2 \%$ to the total cross-section [37].

One phenomenological view of the hadronic cascade is the spallation model [37], consisting of fast and slow components. In the spallation model, the hadronic shower is described by the break up of nuclei by fast hadrons, with the production of secondary particles. The hadronic shower is induced by the interaction of the hadron with the nucleons in the nuclei of the material. The scattered nucleon can then transfer energy to nucleons within the same nucleus, creating a fast intranuclear cascade, on the order of $\sim 10^{-22}$ s. Additional hadrons may also be formed with enough energy to escape the nuclear potential and contribute to the hadronic shower development.

Other hadrons can stay within the nucleus and transfer their kinetic energy to the nucleus, thereby raising the nucleus to an excited state. The de-excitation of 
the nucleus is known as the slow evaporation stage. The de-excitation process occurs through the emission of nucleons and photons until the nucleus returns to the ground state.

During the creation of hadrons in the cascade, neutral pions $\left(\pi^{0}\right)$ will be created. The decay mode of the $\pi^{0}$ is almost exclusively into two photons, occurring $98.8 \%$ of the time [6]. The production of the two photons leads to an electromagnetic component of the hadronic shower through the processes described in Section 3.1.1, while the production of $\pi^{ \pm}$, n, etc., creates the purely hadronic component of the cascade. The average fraction of the shower which is electromagnetic is subject to large event-by-event fluctuations and is dependent on the energy of the incident particle. Different parameterizations for the energy dependency are available $[37,38]$. In both parameterizations, the electromagnetic component of the shower increases as the incident hadron energy increases, due to the fact that a higher incident energy produces more interactions and thus a higher probability of producing neutral pions.

The position of the production of the neutral pions is also subject to large event-by-event fluctuations and neutral pions can be created anywhere in the absorber material. The EM component of the hadronic shower arising from the $\pi^{0}$ requires a much smaller volume to deposit energy than other components of the shower carrying the same energy, meaning that the energy density is larger in areas of the absorber near $\pi^{0}$ production. Because of this, the hadronic energy profiles will directly reflect the large fluctuations in both the energy and position of the EM component of the shower.

The intrinsic length scale of the hadronic shower is characterized by the nuclear interaction length, $\lambda$, defined as the average distance a high energy hadron will travel in a material before a nuclear interaction occurs. The nuclear interaction length can 
be estimated using

$$
\lambda \simeq 35 \frac{A^{1 / 3}}{\rho} \quad[\mathrm{cm}] .
$$

As an example, the interaction length for copper is $15.59 \mathrm{~cm}$ while it is $10.37 \mathrm{~cm}$ for tungsten.

Hadronic showers are generally broader than EM showers. The secondary hadrons are usually produced with a transverse energy comparable to the energy lost in $1 \lambda$ in most materials [39]. Therefore, the electromagnetic component of the shower provides a pronounced core with a characteristic transverse scale of $R_{M}$, while the purely hadronic component provides a halo with characteristic transverse scale of $\lambda$.

\subsubsection{Sampling Calorimeters}

In a sampling calorimeter, the absorbing material is different from the medium used to generate the signal. The absorber material is usually a dense material with high atomic number allowing for shower development and containment within reasonable spaces. The active medium is usually a gas, a scintillating material or a liquified noble gas such as argon or krypton. The most popular sampling calorimeter structure is parallel plates of absorbing material interleaved with gaps of active material. However, the cylindrical design shown in Figure 3.4 is used in the ATLAS Forward Calorimeter where copper and tungsten are used as absorbing materials while a thin gap of liquid argon is the active medium. The basic structure of the electrode consists of two concentric metal cylinders separated by a potential difference with liquid argon filling the gap between the cylinders.

The signal generated using liquid argon is through the direct collection of free charges created from the process of ionization [37]. When a charged particle 


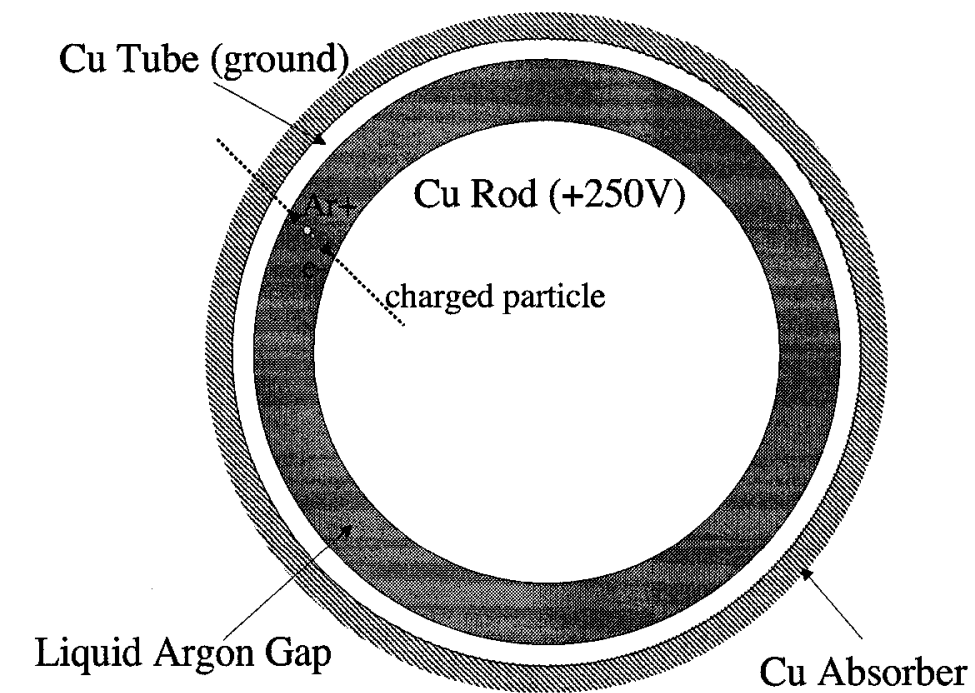

Figure 3.4: Electrode structure for a sampling calorimeter.

crosses the liquid argon gap, it ionizes the atoms. The applied electric field forces the electrons and argon ions to the anode (rod) and the cathode (tube), respectively. The charge from the electrons collected on the electrodes forms the signal.

Since the signal is based on the ionization of the liquid, the response of the calorimeter will be different for incident electrons, photons and hadrons. In the EM showers created by incident electrons or photons, all the energy is eventually deposited through the ionization process. The energy deposited in the active material can therefore be converted into a measureable signal which is linearly dependent on the energy of the incident particle. In contrast, incident hadrons have shower components which lose energy through nuclear interactions which cannot be converted into a useable signal. Furthermore, the varying fraction of energy sharing between the EM and hadronic shower components leads to a non-linear response of the calorimeter.

One important quantity in describing sampling calorimeters is known as the sampling fraction $(S)$ and it is defined [36] as the energy deposited by a minimum ionizing particle (mip) in the active layers of the calorimeter, $E_{v i s}$, relative to the 
total energy deposited anywhere in the calorimeter, $E_{d e p}$ :

$$
E_{v i s}=S E_{d e p}
$$

The differences between mips and particles such as the electron have implications on the value of the sampling fraction [37]. For example, the electron sampling fraction will be lower than the ideal mip value due to the fact that a significant fraction of the electromagnetic shower energy is deposited by low energy $(<1 \mathrm{MeV})$ electrons produced through Compton scattering and the photoelectric effect. The production of these electrons occurs mostly in the absorber. Since the range of the electrons is typically shorter than the distance between active layers, these electrons will only contribute to the signal if they are produced close to the boundary of the active and absorber material, resulting in a lower value of the sampling fraction. Since more low energy electrons are created in the later stages of the shower, the sampling fraction is not constant along the development of the shower and the linearity of the signal depends largely on the containment of the shower.

Another important quantity of calorimeters is the energy resolution. It is the precision to which the energy of an incident particle can be measured and it is defined as the ratio of the width of the energy distribution to the energy, $\sigma / E$. The energy dependence of the resolution is parameterized as

$$
\frac{\sigma}{E}=\frac{a}{\sqrt{E}} \oplus \frac{b}{E} \oplus c
$$

where $E$ is the energy of the particle, $\sigma$ is the variance of the energy distribution, a, $\mathrm{b}, \mathrm{c}$ are constants and $\oplus$ represents addition in quadrature.

The first term in Equation (3.6) is known as the sampling term. In electromagnetic calorimeters, it is usually dominated by the sampling fluctuations. In 
hadronic calorimeters, however, additional large fluctuations arise from the energy sharing between shower components, leading to deviations from the $E^{-1 / 2}$ dependence. Hadronic calorimeters have been designed to maintain equal EM and hadronic shower components, thus eliminating the latter type of fluctuations and approximately restoring the $E^{-1 / 2}$ dependence [37]. These calorimeters are referred to as compensating calorimeters. All ATLAS calorimeters are non-compensating. The second term is known as the noise term and is comprised of both electronics noise and pileup noise. The third term accounts for calibration errors, imperfect quality of detector construction and leakage of energy out the sides and back of the calorimeter. This term is independent of energy and will dominate at high energies.

\subsection{The ATLAS Forward Calorimeter}

A major focus of the ATLAS experiment is physics with large missing transverse energy $\left(\mathbb{E}_{T}\right)$. Events give rise to missing energy when weakly interacting particles such as neutrinos or the proposed lightest supersymmetric particle (LSP) escape the detector without producing an appreciable signal. Their presence in the final state of the interaction, however, can be inferred from the imbalance of momentum in the plane transverse to the collision axis.

An important background for such physics studies is ordinary events in which a particle jet escapes the detector undetected. Jets could potentially escape the detector in the forward region, near the beam hole. The ATLAS Forward Calorimeter suppresses this background by providing calorimetric coverage and therefore particle jet detection in the pseudo-rapidity region of $3.2<|\eta|<4.9$.

The FCal is also important for tagging high-energy forward jets which arise 
from events producing the Higgs boson via Vcctor-Boson-Fusion. For such events, the calorimeter design requirements for transverse energy resolution were set to $\Delta E_{T} / E_{T}<10 \%$ for $E_{T}>25 \mathrm{GeV}$ [40]. Below this energy threshold, jets cannot be tagged at the design luminosity due to pileup effects. The above transverse energy requirement implies that the FCal energy and angular resolutions must be better than approximately $7 \%$ for energies above $250 \mathrm{GeV}$.

At the LHC design luminosity, $L=10^{34} \mathrm{~cm}^{-2} \mathrm{~s}^{-1}$, the forward region of the ATLAS detector will be bombarded with low $p_{T}$ particles, creating a low level of ionization in the calorimeters during each bunch crossing. This requires the FCal to be radiation hard to ensure the stability of the detector over the lifetime of the experiment. As an example, approximately $7 \mathrm{TeV}$ of energy is deposited into the FCal every 25 ns [41]. The ionization dose varies in the FCal from approximately $100 \mathrm{kRad}$ to about $0.5 \mathrm{GRad}$ per LHC year and the flux of neutrons with energies above $100 \mathrm{keV}$ ranges from $10^{4}-10^{6} \mathrm{kHz} / \mathrm{cm}^{2}[40]$.

The ATLAS detector contains two forward calorimeters, FCalA and FCalC, located on either side of the interaction point. Figure 3.5 is a schematic diagram illustrating a cross-sectional view of the upper half of the FCal within the cryostat and in relation to the other calorimeters. The $Z$-axis represents the LHC beam axis. The front face of each FCal is located approximately $4.7 \mathrm{~m}$ from the interaction point and they are both housed in the end-cap cryostats, along with the electromagnetic calorimeters (EMEC) and hadronic end-cap calorimeters (HEC). Each FCal is composed of three modules. FCal1, the module closest to the interaction point, is considered an electromagnetic calorimeter, while FCal2 and FCal3 are both hadronic calorimeters. The three modules, along with the copper alloy block located just behind the FCal, serve to provide ample shielding and a source of background reduction 


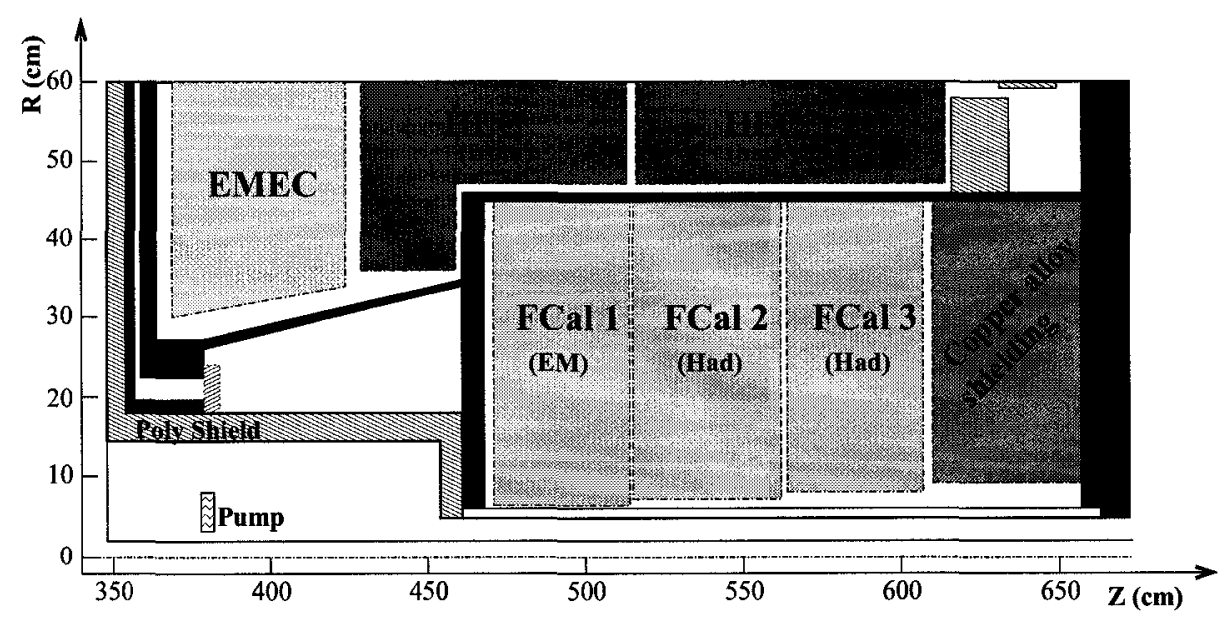

Figure 3.5: Schematic diagram of the FCal in relation to the other calorimeters within the end-cap cryostat.

for parts of the muon system directly behind the calorimeters and near the beam pipe.

Each of the three modules is a liquid argon ionization, sampling calorimeter. Choosing liquid argon for the FCal permitted the integration of the FCal with the other calorimeters within the same cryostat. The three detectors (HEC, EMEC, FCal) within a common cryostat complete the hermetic design of the end-cap region of the ATLAS calorimeter system.

While liquid argon is the active material for all three modules, the absorbing material changes. In the electromagnetic calorimeter (FCal1), copper is used as the absorbing material while the hadronic components (FCal2/3) employ tungsten as the absorbing material. The physical properties of each module are listed in Table 3.1. Each module has an outer radius of $449.6 \mathrm{~mm}$ with a depth of approximately $440 \mathrm{~mm}$. Thus, FCal1 is $28 X_{0}$ or $2.7 \lambda$ deep, while FCal2 is $3.7 \lambda$ deep and FCal3 is $3.6 \lambda$ deep.

Copper was chosen for the absorber matrix of FCal1 for two primary reasons: 1) the Molière radius $(15.3 \mathrm{~mm})$ is large enough so that the size of the electromagnetic 


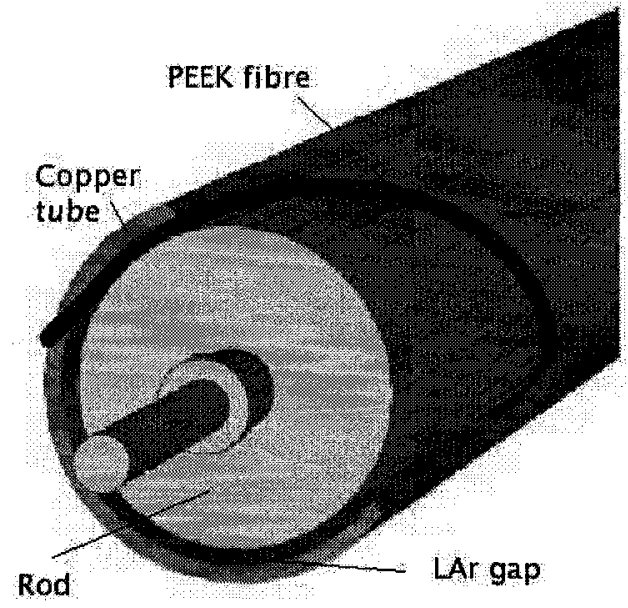

(a) FCal Electrode

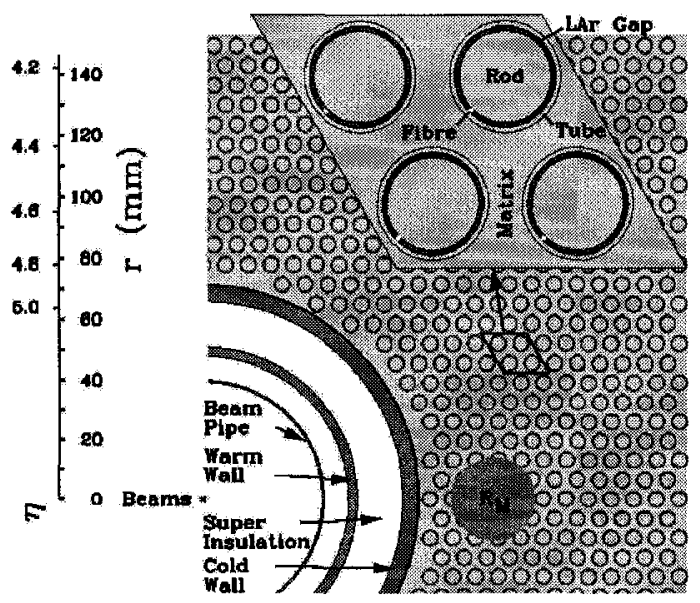

(b) Front Face of FCal1

Figure 3.6: (a) Electrode structure for the FCal and (b) front face of FCal1, with the electrodes embedded into the absorber matrix.

shower is large compared to the electrode spacings, ensuring that the response across the front face is reasonably uniform, 2) copper allows the heat deposited in the module (primarily from minimum-bias particle showers) to be removed efficiently and avoid large temperature differentials which could cause the liquid argon to boil.

Tungsten was chosen as the absorber matrix for FCal2/3 to limit the longitudinal and transverse spreading of hadronic showers and also to limit the leakage into calorimeter cells at lower $\eta$ where particle energies are typically lower and could be overwhelmed.

Due to the harsh conditions in the forward direction of the ATLAS detector mentioned above, the FCal is not a traditional parallel plate sampling calorimeter. Rather, the detector utilizes the novel electrode structure based on concentric cylindrical rods and tubes, as shown in Figure 3.6a. The electrodes are produced by inserting cylindrical rods into copper tubes with liquid argon filling the gap in be- 
tween the rods and tubes. The rod maintains a positive potential while the tube is held at ground. The current of electrons drifting toward the rod constitutes the signal [42].

Each module consists of a repeating pattern of electrodes, organized as shown in Figure 3.6b. The schematic diagram illustrates the front face of FCall. The diagram clearly shows the electrode structure as it is embedded in the absorber matrix of the FCal. The pattern of cylindrical electrodes gives the FCal fine transverse segmentation, however, the electrodes run the entire length of the module, leaving limited longitudinal segmentation.

FCal1 has 12260 electrodes, while FCal2 has 10200 and FCal3 has 8224 . Also shown in Figure 3.6b is the fact that the electrodes are grouped into tiles for readout purposes. There are four electrodes per tile in FCal1, while there are six per tile in FCal2 and nine per tile in FCal3. Because of the geometry of the inner and outer edges, there are exceptions to the number of electrodes per tile. The tube groups are further summed, resulting in 1008 readout channels for FCal1, 500 and 254 readout channels for FCal2 and 3, respectively.

The electrodes of each module are aligned parallel to the LHC beam axis, thus the particles will enter the FCal at small angles relative to the electrode axis. Also, due to the non-homogeneity of the front face of the FCal, there is a signal dependence on the impact point of the particle [43].

The liquid argon gaps were chosen to be approximately $1 / 8$ the size of conventional parallel plate sampling calorimeters. The gap sizes for each module are provided in Table 3.1. The unusually small gap sizes were needed to avoid the problem of charge accumulation due to the low mobility of the positive charge carriers in 


\begin{tabular}{c||c|c|c} 
Property & FCal1 & FCal2 & FCal3 \\
\hline Absorber Matrix Material & Copper & Tungsten & Tungsten \\
\hline Inner Radius $(\mathrm{mm})$ & 72 & 79 & 86 \\
\hline Outer Radius $(\mathrm{mm})$ & 449.6 & 449.6 & 449.6 \\
\hline Length $(\mathrm{mm})$ & 444.2 & 442.3 & 442.3 \\
\hline LAr Gap $(\mu \mathrm{m})$ & 250 & 375 & 500 \\
\hline Interaction Length $(\lambda)$ & $2.7\left(\right.$ or $\left.28 \mathrm{X}_{0}\right)$ & 3.7 & 3.6 \\
\hline No. Electrodes & 12260 & 10200 & 8224 \\
\hline No. Electrodes/Tile & 4 & 6 & 9 \\
\hline No. Readout Channels & 1008 & 500 & 254
\end{tabular}

Table 3.1: Properties of the three ATLAS FCal modules.

argon. In regions of high ionization, large levels of charge accumulation can distort the electric field causing degradation in the signal [44]. The small gaps of the FCal protect against large levels of charge accumulation while also providing a faster signal. Gaps of these dimensions are difficult to maintain in parallel plate calorimeters, so the cylindrical electrode structure was chosen for the FCal.

The gap sizes increase in FCal2 and FCal3 since the ionization density from the showers is lower than in FCal1. The gaps between the rods and tubes are maintained by non-conductive PEEK (polyetheretherketone) fibres which are helically wound around the rod, as shown in Figure 3.6a.

The electrodes in the hadronic modules consist of the copper tube containing the tungsten rod with an anode pin attached to the end. The electrodes are surrounded by a large number of tungsten slugs which comprise the absorber matrix, filling the space between the copper tubes. The $1 \mathrm{~cm}$ long slugs are arranged in a 


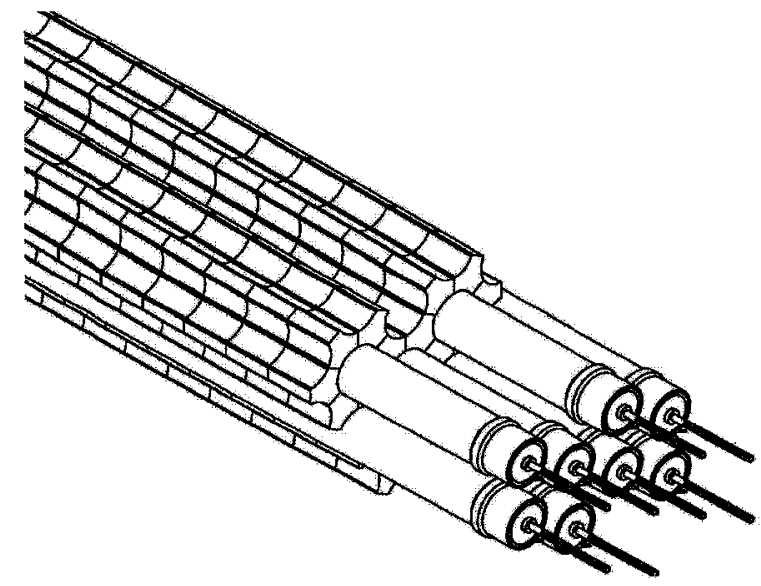

Figure 3.7: Diagram of the electrode and absorber matrix structure in FCal2 and 3.

hexagonal pattern, as illustrated in Figure 3.7. The density for the tungsten rods is $19.2 \mathrm{~g} / \mathrm{cm}^{3}$ while the tungsten alloy slugs (WFeNi), composed of $97 \%$ tungsten powder, $2 \%$ nickel and $1 \%$ iron by weight, have a density of $18.3 \mathrm{~g} / \mathrm{cm}^{3}$.

A $23.5 \mathrm{~cm}$ thick copper plate is situated at each end of the hadronic modules, separated by copper cladding plates located at the inner and outer radii. Together, the endplates and cladding plates form the mechanical structure of the modules. Each endplate has 10200 (8224) through holes to accommodate the electrodes for FCal2(3). The overall structural integrity of the modules is formed by the combination of the endplates, the cladding plates and the copper tubes. Further strength is added to the modules with the insertion of the rigid tungsten rods.

\subsection{FCal Beam Test 2003 and Simulation}

In 2003, a beam test [45] was performed on the forward calorimeter by the ATLAS FCal group, to both understand the intrinsic properties of the detector and to study 
its performance under ATLAS conditions. The studies included the energy calibration as well as investigations of the response of the FCal with varying energies. In particular, both the linearity and the energy resolution of the FCal were measured as functions of energy. The beam test took place at the $\mathrm{H} 6$ beamline at CERN and included the study of both electrons and hadrons with known energies between $(10-200) \mathrm{GeV}$.

In order to maximize the understanding of any detector, Monte Carlo simulations are used to help describe the physical processes that occur when particles interact with the system. For the simulation of the FCal beam test, the geometry descriptions employ the ATLAS software, GeoModel, and the particle interactions within the detectors are simulated using Geant $4^{1}$. Both the GeoModel and Geant4 software are embedded into the ATHENA computing framework described in Section 2.2.5. Although "stand-alone" simulations of the FCal have been used in the past by the FCal group, this is the first implementation of the beam test simulation within the ATHENA framework. The simulation design of the FCal had been implemented for the final ATLAS detector geometry and was adapted for the specific case of the beam test.

During the beam test, five different beam impact points were targeted on the front face of the FCal, as illustrated in Figure 3.8. The diagram shows the inner and outer edges of the FCal, as well as the relative positions of the beam spots. Positions $1,2,3$ ( $\eta \approx 4.8,4.49,4.42$, respectively, in ATLAS) were used for studies of energy loss near the inner edge of the FCal, while positions $4 \mathrm{H}$ and $4 \mathrm{~L}(\eta \approx 3.64)$ were chosen to maximize the containment of the particle showers. Position $4 \mathrm{H}$ was used to replicate the ATLAS conditions by reproducing the amount of material situated

\footnotetext{
${ }^{1}$ These studies use Geant4.8.3.patch01.atlas00 .
} 


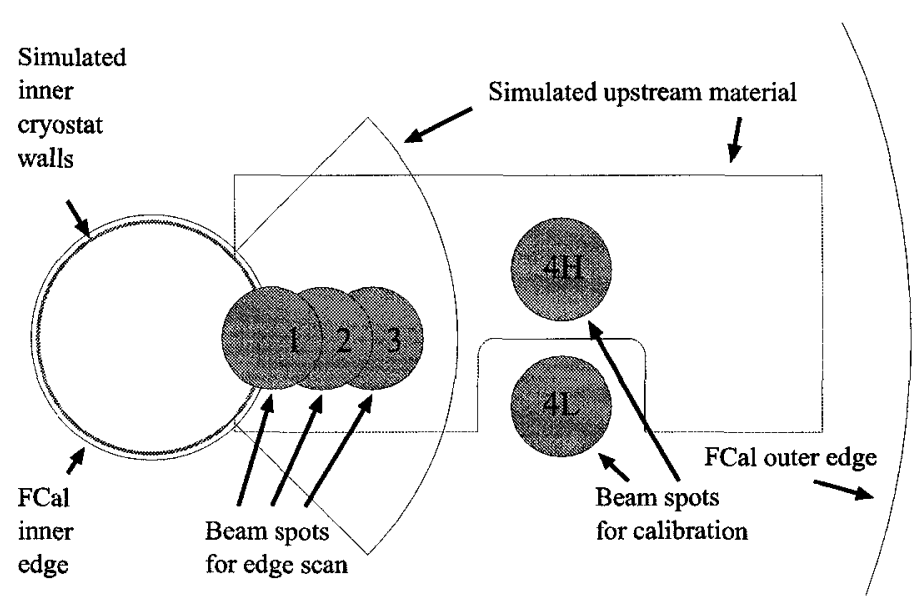

Figure 3.8: Beam spot positions and material used to reproduce ATLAS dead material for each of the 5 beam test positions.

upstream from the FCal. Thus, two $25.4 \mathrm{~mm}$ thick pieces of aluminum were placed in front of the FCal, illustrated by the rectangular structure in Figure 3.8. The amount of upstream material was minimized for position $4 \mathrm{~L}$ in order to study the intrinsic properties of the FCal. The beam used in the test was $6.5 \mathrm{~cm}$ in diameter, a broad beam used to minimize the impact point dependence. The results for the data of position $4 \mathrm{~L}$ can be found in [45].

A schematic diagram of the experimental setup for the beam test is provided in Figure 3.9. B9 represents the magnet system used to steer the charged particle beams in the vertical direction and was located approximately $32 \mathrm{~m}$ upstream from the FCal modules. The beamline instrumentation consisted of beam positioning chambers (BPCs), scintillators $\left(\mathrm{S}_{1}, \mathrm{~S}_{2}, \mathrm{~S}_{3}\right.$, veto counter, muon counter) and a tail catcher. The BPCs were used to measure the beam coordinates, while the scintillators upstream from the FCal were used as triggers. The tail catcher, composed of alternating layers of iron and scintillators, was used to monitor energy leakage out of the back of the FCal. A beam stop, made of iron and concrete blocks was placed behind the tail 


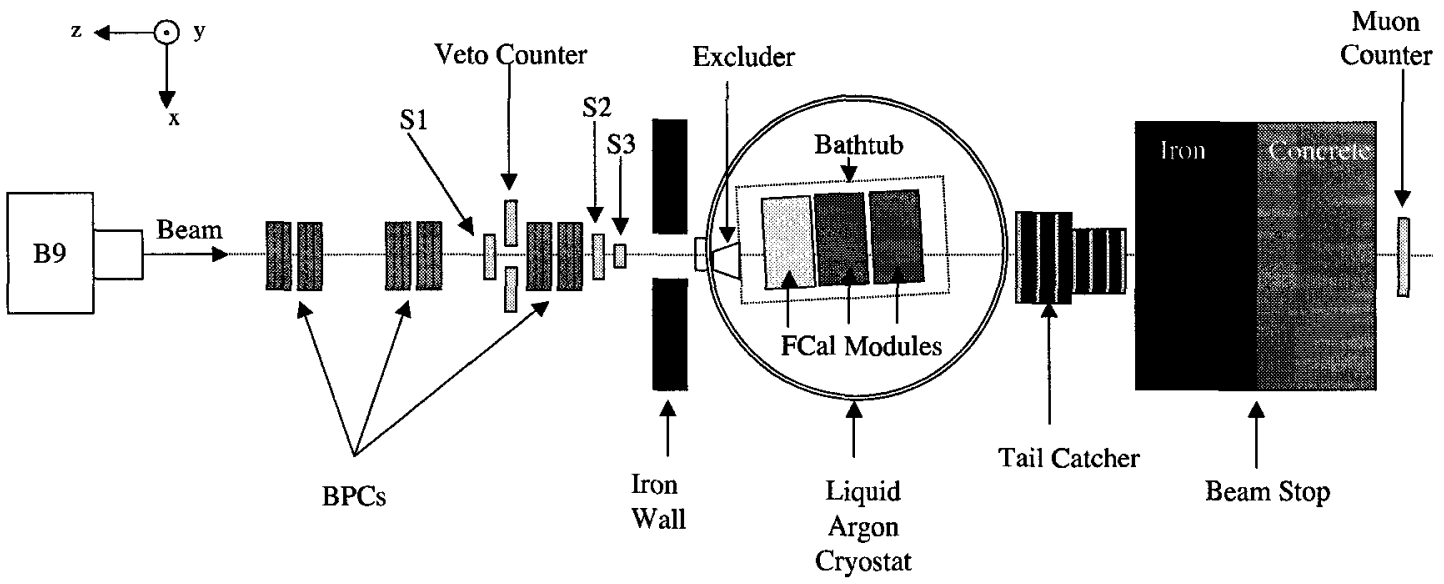

Figure 3.9: Schematic diagram of the beamline setup for the 2003 test.

catcher. The beam line materials implemented in the simulation are the scintillators, the iron walls, the beam stop and the cryostat, which houses the FCal modules. Also added to the simulation, but not illustrated in Figure 3.9 are the three sections of evacuated beam pipes situated between the B9 magnet and the FCal. The total length of the evacuated beam pipe is $16.93 \mathrm{~m}$. In the current setup, the beamline materials in the simulation only act as dead material in front of the FCal. In particular, their readout systems are not simulated, so exact information of the energy deposited in each is not available.

The $\mathrm{H} 6$ cryostat consists of a series of concentric iron cylinders with the long (symmetrical) axis along the $y$-axis of the beam test coordinates ${ }^{2}$. The cryostat has the ability to rotate about the $y$-axis and translate about the $x$-axis. These transformations, along with the ability to steer the beam with the B9 magnet system provide the mechanisms necessary to impact the FCal for all 5 positions shown in Figure 3.8. The dimensions of the cryostat, along with the contents implemented in

\footnotetext{
${ }^{2}$ The coordinate system used is based on the ATLAS coordinate system defined in Section 2.2.
} 


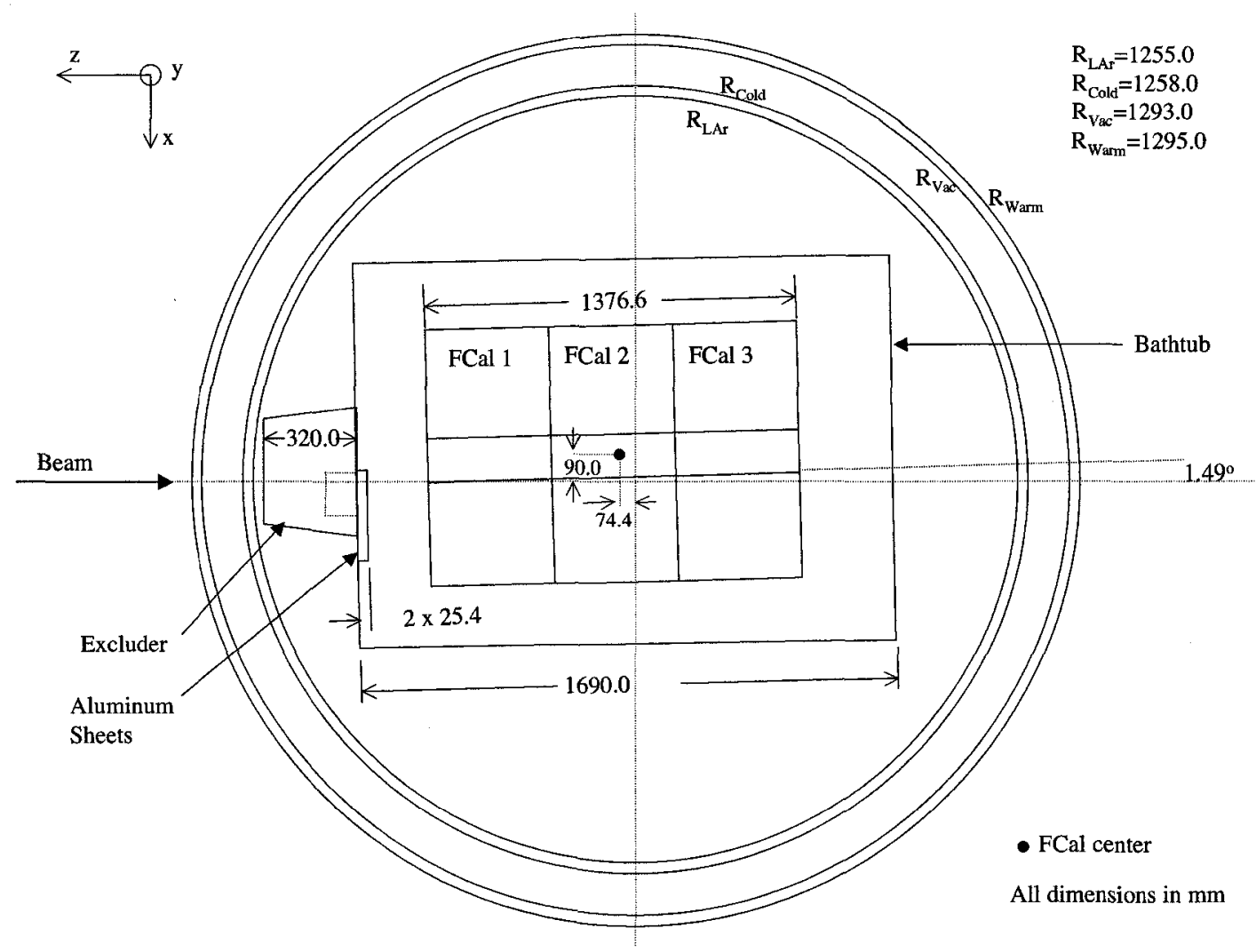

Figure 3.10: Schematic diagram for the contents of the cryostat for the 2003 test beam simulation.

the simulation, are illustrated in Figure 3.10. The space between the cold and warm walls is evacuated while the cryostat is filled with liquid argon. Within the cryostat, the FCal is placed inside a stainless steel bathtub.

The thickness of the bathtub wall is $0.0079 \mathrm{~mm}\left(1 / 16^{\prime \prime}\right)$ and the inside of the front face of the bathtub holds the two aluminum sheets used to reproduce the dead material (cryostat bulkhead) in the ATLAS configuration. The aluminum sheets are both $25.4 \mathrm{~mm}$ thick, with $100 \times 55 \mathrm{~mm}^{2}$ slits such that each of the beam spots are covered by aluminum, except in the $4 \mathrm{~L}$ position.

A liquid argon excluder, composed of rohacell-11 [46] is placed in the bathtub 


\begin{tabular}{c|c|c|c|c} 
Position & $\begin{array}{c}x \text {-Translation } \\
(\mathrm{mm})\end{array}$ & $\begin{array}{c}x \text {-Position } \\
(\mathrm{mm})\end{array}$ & $\begin{array}{c}\text { Rotation } \\
\alpha\left(^{\circ}\right)\end{array}$ & $\begin{array}{c}\text { Total Rotation } \\
\left(^{\circ}\right)\end{array}$ \\
\hline \hline 1 & $18.0-h \sin \alpha$ & -72.0 & -0.61 & 0.88 \\
2 & $-14.5-h \sin \alpha$ & -104.5 & -0.24 & 1.25 \\
3 & $-47.0-h \sin \alpha$ & -137.0 & 0.12 & 1.61 \\
$4 \mathrm{H}$ & $-155.0-h \sin \alpha$ & -245.0 & 1.49 & 2.98 \\
$4 \mathrm{~L}$ & $-155.0-h \sin \alpha$ & -245.0 & 1.49 & 2.98
\end{tabular}

Table 3.2: Translation and rotation values for the cryostat used in the simulation, where $h=1295 \mathrm{~mm}$ is the distance from the cryostat pivot point to the front face of the FCal.

to fill the space between the front face of the bathtub and the cryostat wall and has a depth of $z=320 \mathrm{~mm}$. It is trapezoidal in shape such that the full extent of the excluder only reaches to the center of position 4 . There exists a $100 \mathrm{~mm}$ deep slit in the excluder on the face closest to the FCal. The slit is $140 \mathrm{~mm}$ wide in the $x$-direction and only covers positions 1, 2 and 3 of the beam test.

All three FCal modules (the actual FCalC modules of the ATLAS detector) were used in the beam test. The placement of the FCal within the cryostat is illustrated on Figure 3.10. The modules were offset in the cryostat by $-90 \mathrm{~mm}$ along the $x$-axis, $15 \mathrm{~mm}$ along the $y$-axis, $74.4 \mathrm{~mm}$ along the $z$-axis and rotated by an angle of $1.49^{\circ}$ around the $y$-axis. The offset, along with a translation and rotation of the cryostat allowed for the beam to impact the FCal with the same angle as in the ATLAS configuration. The translation and rotation values used for each of the five test beam positions are given in Table 3.2. Because the cryostat was rotated with respect to a pivot point at the back of the cryostat, an extra translation must be performed. 


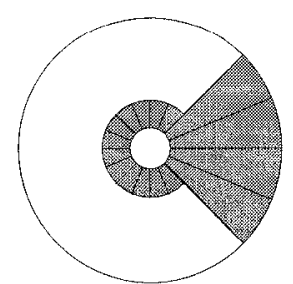

FCal1C

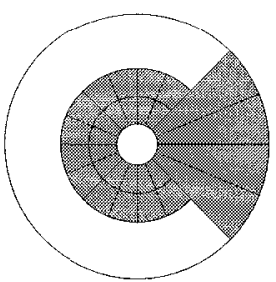

FCal2C

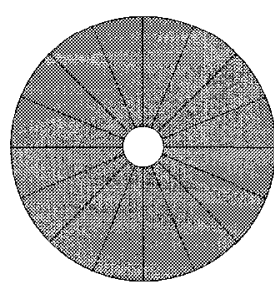

FCal3C

Figure 3.11: Schematic diagram for the readout coverage of the FCal modules for the 2003 test.

The extra translation depends on the distance, $h$, from the pivot point to the front face of the FCal and the angle of the rotation, $\alpha$ :

$$
x=-h \sin \alpha .
$$

In the case of the cryostat used in the beam test simulation, $h=1295 \mathrm{~mm}$. There is also a translation that is needed to compensate for the initial rotation of the FCal with the cryostat, but this is small and is neglected in the simulation.

Due to a lack of cabling and electronics at the time of the beam test, some sections of the FCal were not readout. The readout coverage is illustrated in Figure 3.11. Both FCal1 and FCal2 have $90^{\circ}$ wedges instrumented. The wedges are centered around $x=0$, open in the positive $x$-direction and extend out to the full radius of the module. These sections, along with the annular regions around the beam hole were chosen to provide approximate lateral containment of electrons and pions and to measure energy that may splash across the beam hole during the inner edges studies. The entire FCal3 module was instrumented during the beam test. The energy deposited in the full FCal is available during the simulation of the beam test.

The particle beams in the simulation are created with the particle generator within the ATHENA framework. The user has complete control of where the particles are created. This is exploited in the different studies explored in the next 


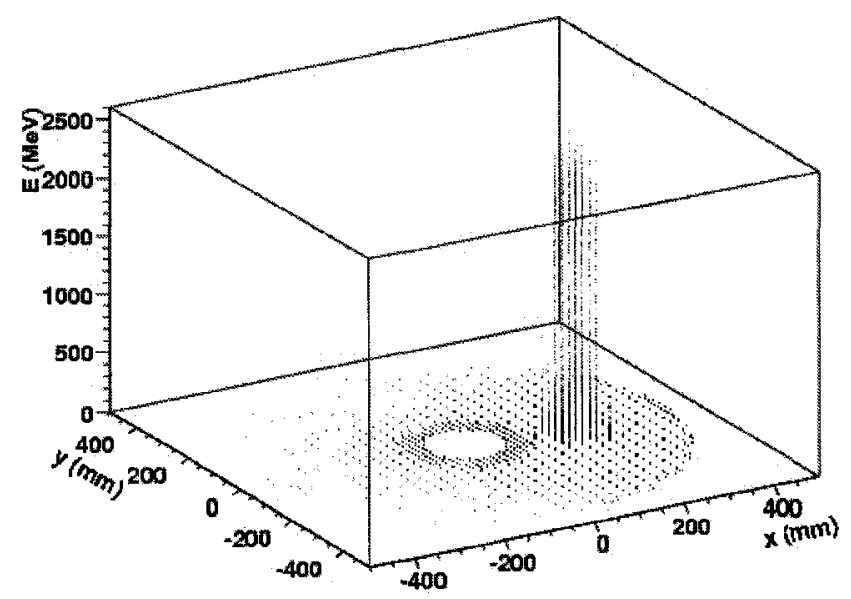

Figure 3.12: Energy deposited in the liquid argon gaps of FCal1 due to a simulated $200 \mathrm{GeV}$ electron beam.

sections. The position of the particle generator for calculating the sampling fractions is different than in the rest of the beam test studies and will be explained fully in Section 3.3.3.

The particles for the beam test simulation are created approximately $32 \mathrm{~m}$ upstream from the cryostat in the z-direction (at the point of B9) and distributed uniformly over a $65 \times 65 \mathrm{~mm}^{2}$ region in order to obtain the broad beam used in the beam test. This creates a beam which allows for impact positions that are evenly distributed across a portion of the face of the FCal. The particles are also generated with varying momentum directions to hit the desired location of the FCal depending on the position being tested. An example of a beam spot is shown in Figure 3.12 for position $4 \mathrm{~L}$. Shown is the energy deposited in the liquid argon gaps of the calorimeter due to $200 \mathrm{GeV}$ electrons. The results are at the simulation level, meaning that noise is not included. 


\subsubsection{Simulation of the FCal Modules}

The modelling of the electromagnetic (FCal1) and hadronic (FCal2,3) modules are performed in different ways. The absorber for FCal1 is modelled as a material mixture of copper and liquid argon. Tubes of liquid argon are added to the matrix, but because the holes in the copper matrix are larger than the copper tubes, liquid argon will fill the gaps between the tubes and the matrix. Thus, the absorber matrix of FCal1 is modelled as a mixture of copper and liquid argon, with mass fractions of $98.9 \%$ and $1.1 \%$, respectively.

For the hadronic modules, cylinders of liquid argon are inserted into the matrix and the tungsten rods subsequently inserted into the liquid argon, leaving the active liquid argon annular gap. The copper tubes are not explicitly created, but are accounted for in the density calculations of the absorber matrix. The volume fractions and resulting matrix densities are given in Table 3.3. As with FCal1, the absorber matrix for the hadronic modules will be simulated as a single volume. However, because of the complicated structure of the tungsten slugs, the material mixture used for the matrix is more involved than in FCal1. The absorber matrix density also includes the copper endplates which accounts for $10.5 \%$ of the total volume of each module. Detailed calculations of the volume fractions can be found in [47]. The differences in $\mathrm{FCal} 2 \mathrm{~A}, \mathrm{C}$ arise from a change in vendor of the tungsten slugs during production, leading to different values in the parameters of the slugs [40]. The material mixture for the absorber matrix is effectively a mixture of the copper endplates, the copper tubes, the tungsten slugs and the inactive liquid argon which fills any gaps between the rest of the material. 


\begin{tabular}{|c|c|c|c|c|c|}
\hline \multirow{2}{*}{ Material } & \multirow{2}{*}{$\begin{array}{l}\text { Density } \\
\left(\mathrm{g} / \mathrm{cm}^{3}\right)\end{array}$} & \multicolumn{4}{|c|}{ Volume Fraction } \\
\hline & & $2 \mathrm{~A}$ & $2 \mathrm{C}$ & $3 \mathrm{~A}$ & $3 \mathrm{C}$ \\
\hline $\mathrm{Cu}$ & 8.96 & 0.151 & 0.143 & 0.143 & 0.143 \\
\hline WFeNi & 18.3 & 0.735 & 0.739 & 0.748 & 0.748 \\
\hline LAr & 1.4 & 0.114 & 0.118 & 0.109 & 0.109 \\
\hline \multicolumn{2}{|c|}{$\begin{array}{c}\text { Matrix Density }\left(\mathrm{g} / \mathrm{cm}^{3}\right) \\
\text { (with endplates) }\end{array}$} & 14.33 & 14.34 & 14.45 & 14.45 \\
\hline
\end{tabular}

Table 3.3: Material properties used for the simulation of the FCal hadronic modules. Included are the volume fractions of each material and the overall calculated densities of the absorber matrix.

\subsubsection{Geant4 Physics Lists}

Within Geant4, different physics lists are available which contain the physics processes used in the simulation of particle interactions, according to the model describing them. For example, two of the physics lists studied by the ATLAS collaboration are QGSP_BERT and QGSP_EMV [48]. Both are based on the quark gluon string model for high energy hadronic interactions of protons, neutrons, pions and kaons. In QGSP_BERT, low energy $(<10 \mathrm{MeV})$ hadronic interactions are calculated using the Bertini cascade $[49,50]$, while the low energy parameterized model (LEP) [33] is used in QGSP_EMV.

The electromagnetic interactions are of major importance to the interaction of an electron incident on the FCal. Both physics lists stated above employ the standard electromagnetic physics processes, containing all the interactions described in Section 3.1.1; however, QGSP_EMV was created for enhanced CPU performance. 
The difference in the two physics lists arises from the treatment of multiple scattering. Both physics lists utilize Lewis theory [51] to describe multiple scattering; however, differences occur with the simulation of particles entering new volumes. The implementation of multiple scattering in QGSP_EMV does not limit the particle's range (as in all other physics processes) after entering a new volume, decreasing the accuracy in the simulation of back-scattering events for low energy particles. The low energy particles penetrate too deeply into the new volume and, due to energy loss, cannot reach the boundary in the backward direction, affecting the amount of energy deposited in the active regions of sampling calorimeters. The effects of both physics lists will be explored in terms of the electron sampling fraction as well as the energy resolution of the FCal in Section 3.3.3.

\subsubsection{FCal Simulation Performance}

\subsubsection{FCal Sampling Fractions}

As described in Section 3.2, the FCal is a sampling calorimeter, therefore only the ionization energy which is deposited in the active material (LAr) will contribute to the signal. Thus, a conversion factor must be used to reconstruct the total energy deposited in the FCal from the energy deposited in the liquid argon gaps due to the initial particle. The value used for this conversion factor for incident electrons is called the electron sampling fraction, as defined in Section 3.1.3. The sampling fraction was calculated for the ATLAS FCal using the simulation software explained above.

The simulation of the ATLAS subdetectors provides information about energy deposition for all regions. In particular, the energy deposited in the active material 


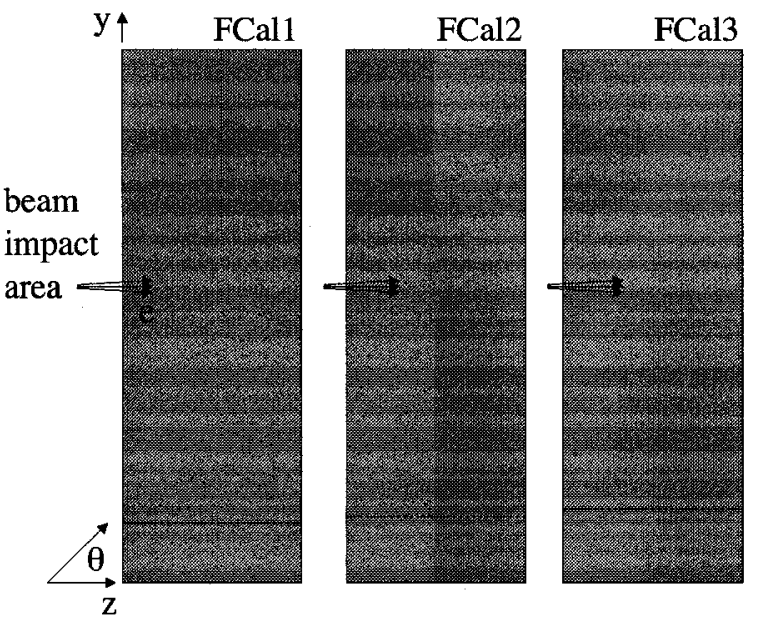

(a) Side View FCal1,2,3

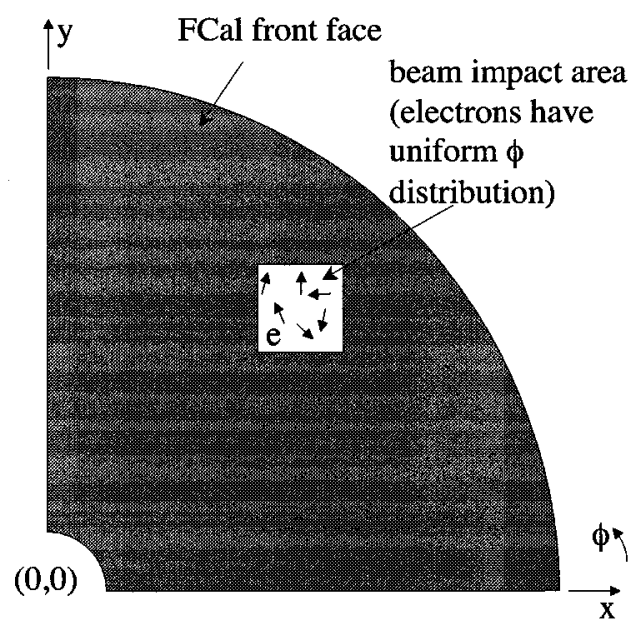

(b) FCal Front Face

Figure 3.13: Geometry setup for the calculation of the FCal sampling fraction.

and absorber regions of the FCal can be calculated. Furthermore, the type of interaction causing the deposition is also known. This information can be used to calculate the sampling fraction. It can be obtained using the formula

$$
S=\frac{E_{\text {Active| }}{ }_{\text {Ion }}}{E_{\text {Active| }\left.\right|_{\text {Tot }}}+E_{\text {Abs }}}
$$

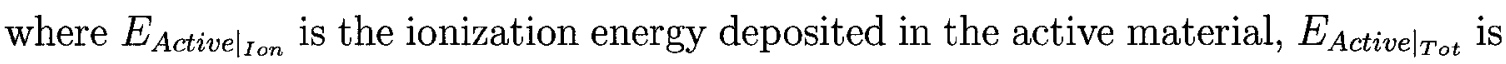
the total energy deposited in the active material and $E_{A b s}$ represents the total energy deposited in the absorber material.

To calculate the sampling fraction, electrons were generated directly in front of each of the modules to avoid any interactions with material existing in front of the FCal. This is illustrated in Figure 3.13a. In each case, 1000 electrons of varying energies from (20-200) GeV were directed at the FCal. As shown in Figure 3.13b, the particle generator was placed at different $x, y$-positions in front of the FCal. The generator position was evenly distributed over the region $205 \mathrm{~mm}<x, y<295 \mathrm{~mm}$ 


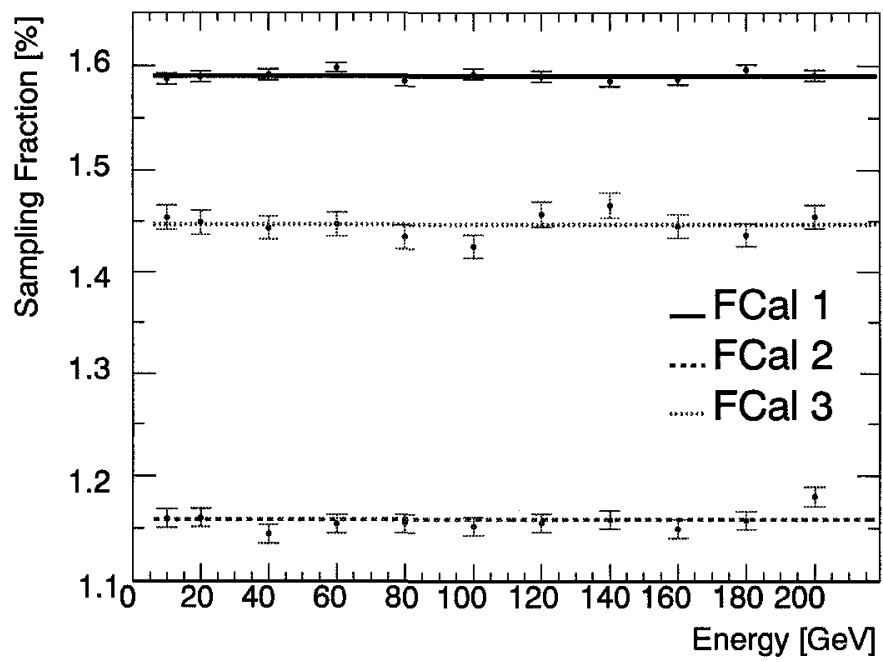

Figure 3.14: Energy dependence of electron sampling fractions for the FCal modules along with linear fits.

to ensure that the electron showers were maximally contained and to minimize the impact point position dependence mentioned in Section 3.2. In addition, the particles were produced at small polar angles $\left(\eta^{\prime}=[3.5,3.8], \phi^{\prime}=[0,2 \pi]\right.$, where the primes refer to the fact that these quantities are measured about the particle generator axis and not the beam axis) to ensure that the particle showers developed as they would in the ATLAS configuration (i.e. as if generated at the interaction point).

The calculated sampling fraction for incident electron energies of (20-200) GeV is shown in Figure 3.14 for all three FCal modules, using the QGSP_BERT physics list. As expected, the sampling fraction is energy independent. This energy independence is maintained between the different Geant4 physics lists described above; however, the absolute value of the sampling fractions changes for the modules. The final value of the sampling fraction is obtained from the linear fit to the simulated data shown in Figure 3.14. The values for all three FCal modules using both QGSP_EMV 


\begin{tabular}{|c|c|c|c|c|}
\hline FCal Module & Physics List & $\begin{array}{l}\text { Absorber Density } \\
\qquad\left(\mathrm{g} / \mathrm{cm}^{3}\right)\end{array}$ & \multicolumn{2}{|c|}{$\begin{array}{l}\text { Sampling Fraction } \\
\qquad \%)\end{array}$} \\
\hline \multirow[t]{2}{*}{1} & EMV & 8.9 & 1.364 & \multirow{2}{*}{ \pm 0.001} \\
\hline & BERT & 8.9 & 1.590 & \\
\hline \multirow[t]{4}{*}{2} & EMV & 15.366 & 0.834 & \multirow{4}{*}{ \pm 0.002} \\
\hline & & 14.39 & 0.856 & \\
\hline & \multirow[t]{2}{*}{ BERT } & 15.366 & 1.122 & \\
\hline & & 14.39 & 1.158 & \\
\hline \multirow[t]{4}{*}{3} & \multirow[t]{2}{*}{ EMV } & 15.366 & 1.067 & \multirow{4}{*}{ \pm 0.003} \\
\hline & & 14.39 & 1.111 & \\
\hline & \multirow[t]{2}{*}{ BERT } & 15.366 & 1.402 & \\
\hline & & 14.39 & 1.447 & \\
\hline
\end{tabular}

Table 3.4: Sampling fraction results for the FCal modules. The errors are statistical.

and QGSP_BERT are provided in Table 3.4. The amount of energy deposited in the liquid argon gaps is increased when using QGSP_BERT compared to QGSP_EMV, resulting in larger values for the sampling fractions. The increase is approximately $15 \%$ for FCal1 and approximately $25 \%$ for FCal2,3. The results indicate that the different implementations of multiple scattering in Geant4 have a major effect on the electron sampling fractions of the forward calorimeter modules. The cost of using the CPU-enhanced physics list (QGSP_EMV) is to assign systematic uncertainties to the values of the electron sampling fractions.

The density of the absorber material was also shown to affect the values of the sampling fractions. It was particularly important for FCal2,3, where the simulation 
relies on a mixture of liquid argon, copper and WFeNi for the density calculations, as described in Section 3.3.1. Initial estimates of the effective density of FCal2,3 absorbers were $15.366 \mathrm{~g} / \mathrm{cm}^{3}$. The detailed calculations provided in [47] improved on this estimation and the density was later replaced by the densities provided in Table 3.3 of Section 3.3.1. Sampling fraction values calculated using the two densities are provided in Table $3.4^{3}$. As the density of the absorber increases, the sampling fractions decrease, but the relative increase from the QGSP_EMV value to the QGSP_BERT value remains the same $(\approx 15 \%, \approx 25 \%, \approx 25 \%$ for FCal1,2,3 respectively).

\subsubsection{FCal Beam Test Simulation}

An example of an energy distribution in the FCal from the 2003 beam test is provided in Figure 3.15. Distributions for both data and Monte Carlo are given for $193.1 \mathrm{GeV}$ electrons incident on the FCal. Shown are the reconstructed energy for the data (units of ADC counts), and the energy deposited in the liquid argon gaps for the Monte Carlo (units of $\mathrm{MeV}$ ). The data is reconstructed by summing the energy within an $8 \mathrm{~cm}$ cylinder around the impact point on the front face of FCal1 [45].

The low energy tail in the data is due to the presence of pion contamination in the electron beam, a source of background which is not included in the simulation. Attempts were made to model the background using the pion beams of the same energy [45]. The result is shown as the dashed line in Figure 3.15a.

At this stage in the analysis, a direct comparison of the energy resolution between the data and Monte Carlo cannot be made because the detector noise was not added to the simulation and it is known to increase the width of the energy

\footnotetext{
${ }^{3}$ The new density, $14.39 \mathrm{~g} / \mathrm{cm}^{3}$, had to be used for both modules due to a limitation in the simulation software. It was chosen as the average between the densities for FCal2,3.
} 


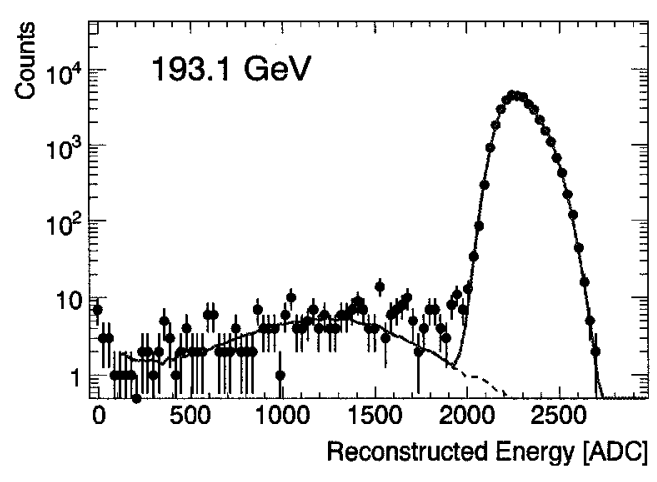

(a) Data

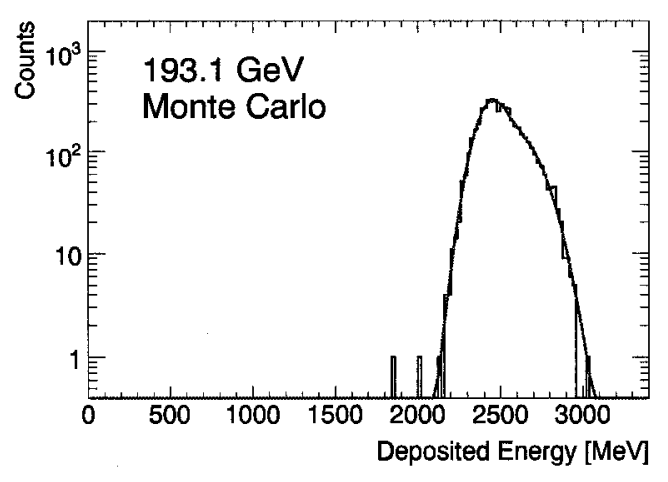

(b) Geant 4

Figure 3.15: $193.1 \mathrm{GeV}$ electron energy distributions. (a) Reconstructed electron energy from data. (b) Energy deposited in the liquid argon gaps calculated by the simulation. Figures from [45].

distribution. However, both the data and the Monte Carlo distributions are fit with a double-Gaussian function. The main peak and the high energy 'shoulder' are present due to the impact point dependence of the FCal [43]. The effect indicates that the energy sampled in the liquid argon gaps differs if the incident electron strikes the liquid argon or the rods/absorbers first. It is reduced in the latter case, due to the higher density of the materials. The range of the secondary particles in the shower is limited, decreasing the possibility to reach the liquid argon gaps. This effect is more pronounced in FCal2 due to the higher density of the tungsten rods compared to the copper rods used in FCal1 [43].

The linearity of the FCal for incident electrons of varying energies is represented in Figure 3.16. For the data, the energy is reconstructed using the $8 \mathrm{~cm}$ cylindrical clustering method described above, while the Monte Carlo energy is reconstructed using the formula

$$
E_{\text {Reco }}=\sum_{i} \frac{E_{F C a l_{i}}}{S_{i}}, \quad i=1,2,3
$$




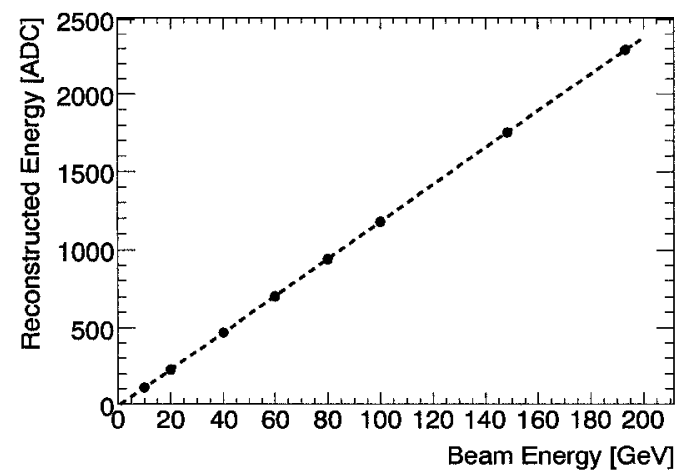

(a) Data: Linearity

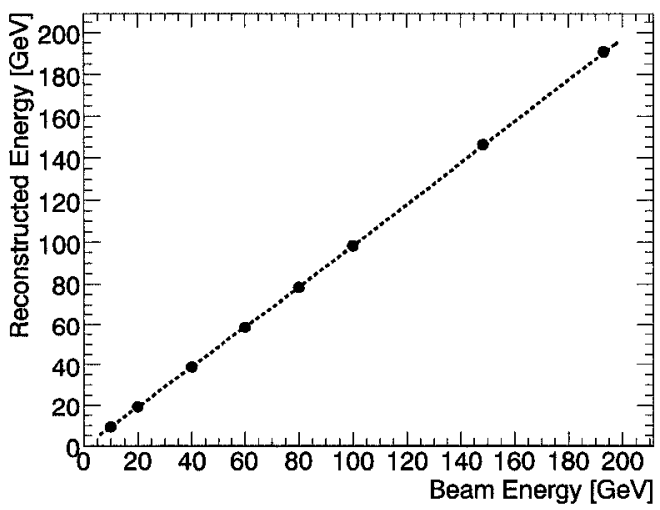

(c) Geant4: Linearity

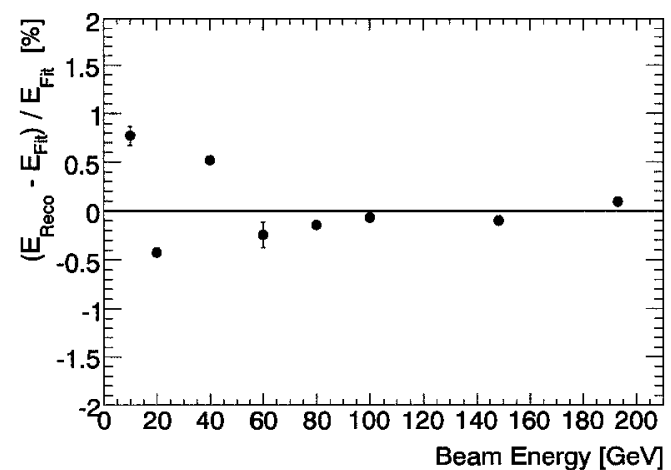

(b) Data: Residuals

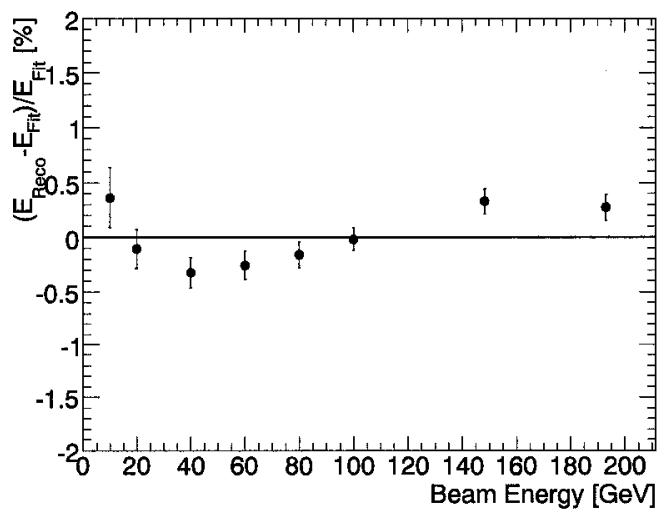

(d) Geant4: Residuals

Figure 3.16: Linearity and residuals for electrons. (a), (b) Data from [45] and (c), (d) Geant4. Only statistical errors are shown.

$E_{F C a l}$ is the energy deposited in the liquid argon gaps and $S$ is the sampling fraction of the three modules, determined in Section 3.3.3.1.

In Figures 3.16a and 3.16c, the reconstructed energy deposited in the FCal is shown as a function of the beam energy, for data and Monte Carlo, respectively. In both cases, a linear fit is applied.

To illustrate the degree of deviation from linearity, the result of the linear fit 


\begin{tabular}{c||c||c|c}
\multicolumn{1}{c||}{} & Linearity & \multicolumn{2}{c}{ Energy Resolution } \\
\cline { 2 - 4 } & Intercept & $\mathrm{a}\left[\% \mathrm{GeV}^{1 / 2}\right]$ & $\mathrm{c}[\%]$ \\
\hline \hline Data & $-12.3 \mathrm{ADC} \approx-1 \mathrm{GeV}$ & $28 \pm 1$ & $3.5 \pm 0.1$ \\
\hline BERT & $-0.54 \mathrm{GeV}$ & $28.8 \pm 0.3$ & $4.06 \pm 0.04$ \\
\hline EMV & $-0.57 \mathrm{GeV}$ & $32.2 \pm 0.3$ & $4.42 \pm 0.02$
\end{tabular}

Table 3.5: Fit parameter results for the electron linearity and resolution for the FCal. Only statistical errors are shown. Data from [45].

is subtracted from the mean of the reconstructed energy to produce the residual:

$$
R=\frac{E_{\text {Reco }}-E_{\text {Fit }}}{E_{\text {Fit }}}
$$

The residuals are provided in Figures $3.16 \mathrm{~b}$ and 3.16d for data and Geant4, respectively. In both cases, the errors shown are statistical only. A discussion on the systematic errors for the data can be found in [45].

The deviations from linearity are within $0.8 \%$ and $0.5 \%$ for the data and the Monte Carlo, respectively. The conversion to energy in the data is determined using the slope of the linear fit, found to be $12.07 \mathrm{ADC} / \mathrm{GeV}$ [45]. The $y$-intercept of the linear fit is provided in Table 3.5. The negative value is interpreted as being energy lost due to interactions of the electrons in material upstream from the FCal. The Monte Carlo underestimates the energy lost, but this is reasonable since not all of the upstream material was simulated. Noticeably absent in the simulation is a Cherenkov radiation detector located upstream from the B9 magnet system.

The energy dependence of the energy resolution of the FCal for incident electrons is illustrated in Figure 3.17 along with the fit to the formula (see Section 3.1.3):

$$
\frac{\sigma}{E}=\frac{a}{\sqrt{E}} \oplus c
$$




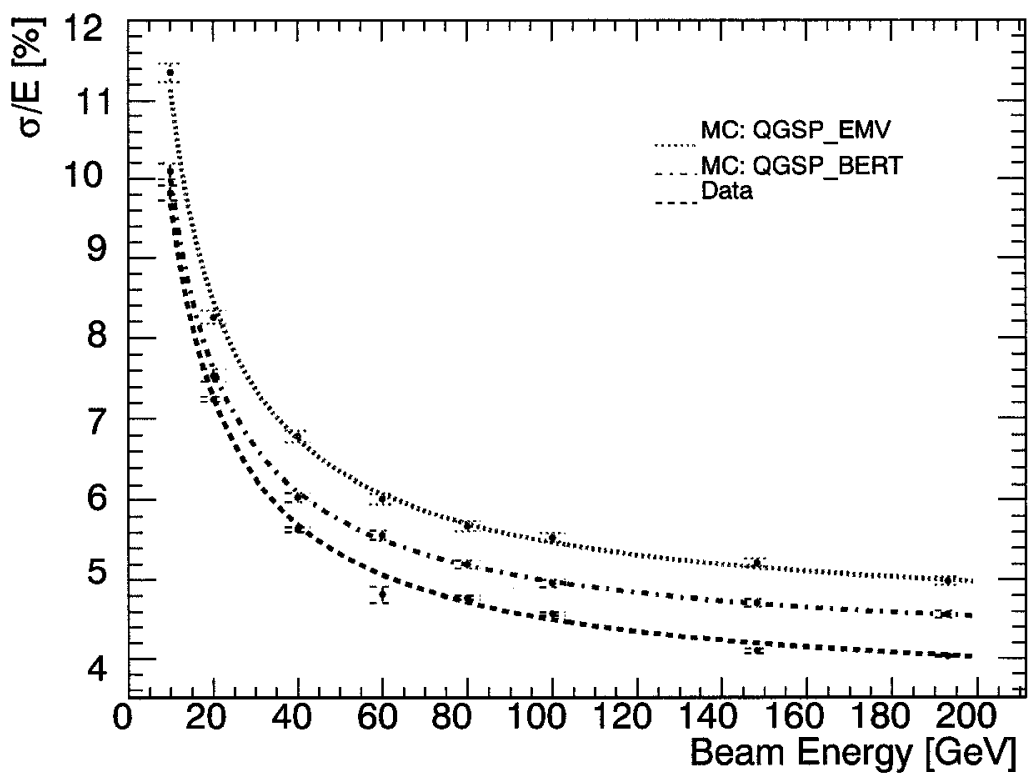

Figure 3.17: Resolution for electrons in the $4 \mathrm{~L}$ position. Data from [45]. The errors are statistical only.

The figure shows the results for the data as well as a comparison to the two physics lists QGSP_EMV and QGSP_BERT. Noise-subtraction was performed on the data, allowing for a fit with Equation (3.11).

The results of the fit parameters are provided in Table 3.5. Historically, the simulations of the FCal provide a worse electron energy resolution than the data [43]. Both QGSP_EMV and QGSP_BERT perform worse than the data; however, improvement is seen with QGSP_BERT. This is not unreasonable, as the increased amount of energy deposited in the liquid argon gaps results in a better energy resolution. Nevertheless, the Monte Carlo energy resolution remains worse than the data for unknown reasons and warrants further investigation. 


\subsection{Summary}

The results from the simulation of electrons provide a starting point to more in-depth analysis of the FCal, using the Monte Carlo toolkit. Having the structure for both the data and the Monte Carlo within the ATHENA framework ensures consistency in the manner in which the analysis is performed.

The sampling fractions calculated using the FCal simulation can now be utilized in the Monte Carlo energy reconstruction chain. This is not only true for the beam test, but for all simulated physics events in the ATLAS detector configuration. Furthermore, the results show that the values are dependent on the Geant4 physics list used, a result that also manifests itself in the performance of the calorimeter simulation in terms of energy resolution. These results can help the ATLAS collaboration sort the advantages and disadvantages to each of the physics list when deciding on a final version of the simulation.

Other studies of the $2003 \mathrm{FCal}$ beam test are ongoing, including the investigation of the $4 \mathrm{H}$ position and FCal inner edge studies (Positions 1, 2 and 3). The Monte Carlo tool provided above will be a valuable resource in these studies. Particularly in the inner edges studies where energy will be lost down the beam hole. With the detailed simulation, the precise position of the energy deposition can be known.

Further work must be done to complete the simulation package within ATHENA. The process of simulating the electronics chain is available in the ATHENA framework but must be validated. The correct noise values obtained from the data need to be implemented for further analysis. The results shown here can be used as reference plots to the simulation performance.

Furthermore, the electron results can be used as a starting point to the analysis 
of hadrons incident on the FCal, studies which are also ongoing but not included in this thesis [52]. The first step in hadronic calibration is usually reconstructing the energy deposition using the electron sampling fractions, highlighting the non-linear response of the calorimeter as explained in Section 3.1.3. 


\section{Chapter 4}

\section{Studies of a Light Stop with the}

\section{ATLAS Detector}

Since the search for supersymmetry (SUSY) is an important objective of the ATLAS collaboration, a SUSY working group was formed, consisting of a subset of the collaboration (of which I am included). The goal of the working group is to facilitate discussions on analysis techniques, results, etc., within the context of supersymmetry studies. In this chapter, we investigate the possible discovery of a light supersymmetric partner to the top quark, as originally proposed by [53]. The purpose of this analysis is to determine whether the search is feasible with the ATLAS detector and warrants further study within the ATLAS SUSY working group.

This chapter begins with an introduction to supersymmetry and a motivation for the existence of a light supersymmetric partner to the top quark. The parameters of the model are then motivated and the calculational methods are provided. Finally, results for both inclusive and exclusive searches are presented. 


\subsection{Supersymmetry}

Supersymmetry (SUSY) $[54,55]$ is one of the favoured theories for new physics phenomena beyond the Standard Model and is a major focus of the ATLAS physics program. Its beginnings as an attempt to combine internal symmetries such as flavour isospin and external symmetries such as Lorentz invariance using fermionic charges [56-60] have lead to many promising theoretical results in the description of particle interactions.

Supersymmetry connects the two categories of fundamental particles (fermions and bosons), relating the matter content of the universe with the particles responsible for the forces of nature. The introduction of supersymmetry requires the existence of new particle content; each of the known Standard Model particles gains a superpartner which has the opposite spin statistics. The superpartners of the fermions are bosons, while the superpartners of the bosons are fermions. In all other aspects, particles and their superpartners are identical. In particular, they have the same quantum numbers and, in an unbroken supersymmetry, identical masses. The properties of the particles and their superpartners (collectively referred to as sparticles) are required to be identical because they belong to supermultiplets.

Supersymmetry contains chiral and vector supermultiplets. The simplest possible chiral supermultiplet contains a single Weyl fermion [55] (a two component spinor with two spin helicity states) and two real scalar fields (which are usually combined to form one complex scalar field). The simplest possible vector supermultiplet contains a spin-1 massless vector boson. The massless vector boson has two helicity states, so its partner must be a massless spin- $1 / 2$ Weyl fermion.

In a supersymmetric extension of the Standard Model, each of the known 


\begin{tabular}{c||c|c} 
Names & spin-0 & spin-1/2 \\
\hline squarks, quarks & $\tilde{Q}=\left(\tilde{u}_{L}, \tilde{d}_{L}\right)$ & $Q=\left(u_{L}, d_{L}\right)$ \\
$(\times 3$ families $)$ & $\tilde{u}_{R}, \tilde{d}_{R}$ & $u_{R}, d_{R}$ \\
\hline sleptons, leptons & $\tilde{L}=\left(\tilde{\nu}_{L}, \tilde{l}_{L}\right)$ & $L=\left(\nu_{L}, l_{L}\right)$ \\
$(\times 3$ families $)$ & $\tilde{l}_{R}$ & $l_{R}$ \\
\hline Higgs, Higgsinos & $H_{u}=\left(H_{u}^{+}, H_{u}^{0}\right)$ & $\tilde{H}_{u}=\left(\tilde{H}_{u}^{+}, \tilde{H}_{u}^{0}\right)$ \\
& $H_{d}=\left(H_{d}^{0}, H_{d}^{-}\right)$ & $\tilde{H}_{d}=\left(\tilde{H}_{d}^{0}, \tilde{H}_{d}^{-}\right)$
\end{tabular}

Table 4.1: Chiral supermultiplets of the MSSM.

particles belongs to either a chiral or vector supermultiplet and must have a superpartner which differs in spin by $1 / 2$ unit. The Minimal Supersymmetric Standard Model (MSSM) [54] is such an extension and contains just enough particle content for a viable theory. The particle content of the MSSM is provided in Table 4.1 for the chiral supermultiplets and in Table 4.2 for the vector supermultiplets.

The left- and right-handed components of the Standard Model fermions have different gauge transformations, so the Standard Model fermions must be members of a chiral supermultiplet. The superpartners of the quarks and leptons, usually called squarks and sleptons, are spin-0 scalar fields. Each of the left- and right-handed pieces of the Standard Model fermions has a scalar superpartner, which is usually denoted with a $(\sim)$. For example, the superpartners of the top quark are called the stop squarks and are denoted by $\tilde{t}_{L}$ and $\tilde{t}_{R}$. The chiral label on the squarks and sleptons reference their Standard Model partners and is not physical since they are spin-0 particles and do not have chirality.

The Higgs boson must also belong to a chiral supermultiplet because it is spin0. Furthermore, a supersymmetric theory requires two complex scalar Higgs doublets, 


\begin{tabular}{c||c|c} 
Names & spin-1/2 & spin-1 \\
\hline \hline gluinos, gluons & $\tilde{g}$ & $g$ \\
\hline wino, $W$-bosons & $\tilde{W}^{ \pm}, \tilde{W}^{0}$ & $W^{ \pm}, W^{0}$ \\
\hline binos, $B$-boson & $\tilde{B}^{0}$ & $B^{0}$
\end{tabular}

Table 4.2: Vector supermultiplets of the MSSM.

a total of 8 degrees of freedom (four complex scalar fields). After electroweak symmetry breaking, 3 degrees of freedom are absorbed by the $W^{ \pm}$and $Z$ via the Higgs Mechanism [15,17], giving rise to their masses. The remaining five degrees of freedom are manifest in the three neutral scalar fields $\left(h, H^{0}, A^{0}\right)$ and two charged scalar fields $\left(H^{ \pm}\right)$. In the MSSM, a non-zero vacuum expectation value is required for $H_{u}^{0}$ and $H_{d}^{0}$ to ensure the success of the Higgs mechanism. The ratio of the two vacuum expectation values $\left(v_{u, d}\right)$ is a free parameter which is traditionally written as

$$
\tan \beta \equiv \frac{v_{u}}{v_{d}}
$$

The value of $\tan \beta$ impacts the mass spectrum of the sparticles but is not fixed by present experiments.

The gauge bosons of the Standard Model belong to vector supermultiplets. The superpartners of the bosons usually have the suffix 'ino' added to the name, as in the case of the Higgsinos and gauginos. The superpartners of the gluons are referred to as the gluinos, while the electroweak symmetry is associated with the vector particles $W^{+}, W^{0}, W^{-}, B^{0}$ and their superpartners $\tilde{W}^{+}, \tilde{W}^{0}, \tilde{W}^{-}, \tilde{B}^{0}$ called winos and bino, respectively. 


\subsubsection{Supersymmetry Breaking}

A spin-0 particle with the same mass as the electron has never been discovered, meaning that if supersymmetry exists, it must be a broken symmetry. As discussed in Section 1.1, gauge invariance forbids masses for vector bosons and fermions within the Standard Model, while allowing masses for scalar fields. Because the superpartners of the Standard Model fermions are scalar particles, they are not forbidden from obtaining masses. Furthermore, the superpartners of the gauge bosons have left- and right-handed components which transform identically under gauge transformations and thus are also allowed to gain masses. Therefore, it is natural for the superpartners to be heavier than the Standard Model particles and it is not unreasonable that they have not been discovered yet. However, if supersymmetry is to help solve the hierarchy problem discussed in Section 1.1, the masses of the superpartners cannot be much more than $\mathcal{O}(1 \mathrm{TeV})$ [61], an energy which will be probed directly at the LHC.

Because the masses for the superpartners do not violate gauge invariance, they can be added 'by-hand'. The parameterization is referred to as 'soft-supersymmetry breaking' since the insertion of the mass terms into the Lagrangian does not reintroduce divergences into the theory. The general parameterization contains scalar masses and gaugino masses, as well as bilinear and trilinear scalar interactions (usually referred to as $B$ and $A$ terms, respectively) [55].

Once supersymmetry and the electroweak symmetry are broken, sparticles with the same quantum numbers can mix [54]. The charged gauginos $\left(\tilde{W}^{ \pm}, \tilde{H}^{ \pm}\right) \operatorname{mix}$ to form two charginos, $\tilde{\chi}_{1,2}^{ \pm}$, while the four neutral gauginos $\left(\tilde{W}^{0}, \tilde{B}^{0}, \tilde{H}_{u}^{0}, \tilde{H}_{d}^{0}\right) \operatorname{mix}$ to form neutralinos, $\tilde{\chi}_{1, \ldots, 4}^{0}$.

Unlike the chiral fermions where the left- and right-handed components must 
have the same mass, the superpartners are scalars with different masses which also mix after the electroweak gauge symmetry is broken. The resulting left-right mixing is mainly important for the third generation and the mass eigenstates are usually labelled as $\tilde{t}_{1,2}, \tilde{b}_{1,2}$ and $\tilde{\tau}_{1,2}[55]$.

\subsubsection{R-Parity}

An important concept in supersymmetric models is known as $R$-Parity [62]. It is a multiplicative quantum number which separates the Standard Model particles and their superpartners because it is defined in terms of spin (s), baryon number (B) and lepton number $(\mathrm{L})$ :

$$
R=(-1)^{2 s+3 B+L} .
$$

All the Standard Model particles have a value of $R=1$, while all the superpartners have $R=-1$ and all terms in the Lagrangian must have $R=1$.

$R$-Parity is conserved in the MSSM, otherwise interactions can occur which lead to proton decay or neutron-antineutron oscillations that are in disagreement with experimental data [55]. Theories do exist with $R$-Parity violation [55], but they will not be discussed here.

With exact $R$-Parity conservation, mixing cannot occur between the particles and the sparticles. Furthermore, every interaction vertex in the theory contains an even number of $R=-1$ sparticles, which has some important phenomenological consequences. The first consequence is that the lightest sparticle, usually referred to as the lightest supersymmetric particle (LSP), must be stable. In addition, colliders must produce sparticles two at a time and each sparticle other than the LSP must decay into an odd number of LSPs. 


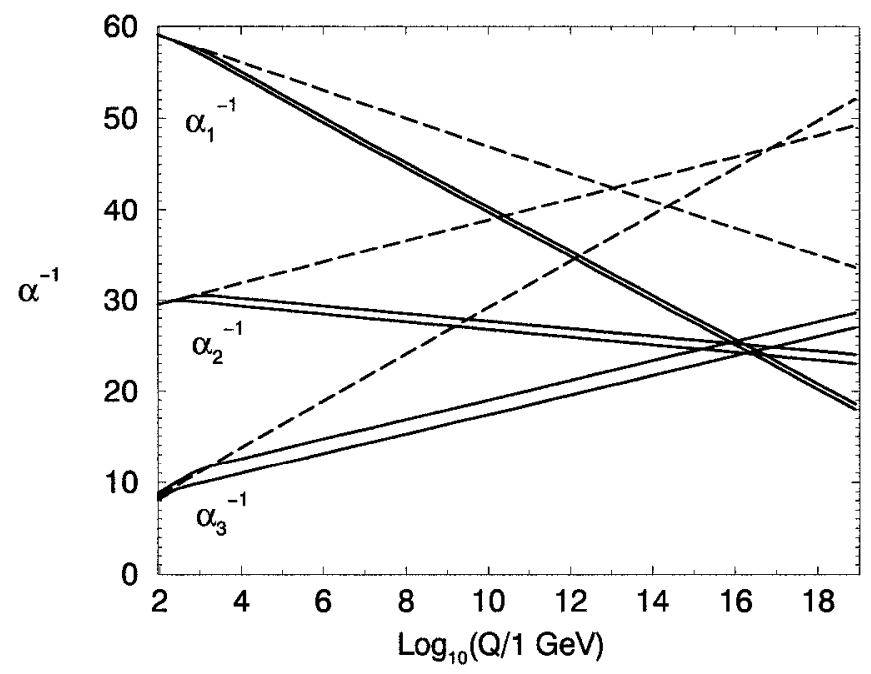

Figure 4.1: Energy dependence of the gauge couplings with Standard Model particle content (dashed) and MSSM particle content (solid). Figure taken from [55].

In many scenarios, the LSP is the lightest neutralino which is electrically neutral and interacts weakly with ordinary matter, therefore establishing an attractive candidate $[63,64]$ for non-baryonic cold dark matter. A direct consequence of a weakly interacting massive particle is that an LSP produced in particle collisions will escape the detector without producing an appreciable signal, leading to signatures of supersymmetry with large amounts of missing energy.

Although supersymmetry has not been discovered directly as of this writing, indirect evidence exists which favours supersymmetry. One promising result is provided in Figure 4.1. The figure illustrates the energy (Q) dependence of the coupling strengths of the Standard Model, known to depend on the particles spectrum available over a given range of energy. The couplings $\alpha_{1,2}$ and $\alpha_{3}$ are proportional to the square of the electroweak and strong coupling constants, respectively. The dashed line is the result if the couplings are calculated only accounting for the Standard Model particle 
content, while the solid line is the result if the couplings are calculated using the particle content of the MSSM. In the case of the MSSM, the sparticle mass thresholds were varied between $250 \mathrm{GeV}$ and $1 \mathrm{TeV}$ while $\alpha_{3}\left(m_{Z}\right)$ was varied between 0.113 and 0.123 [55]. Clearly the couplings do not unify with only the Standard Model particle content while unification occurs in the MSSM scenario.

Both the predictions of the candidate for cold dark matter and the unification of the gauge couplings within a supersymmetric framework give reason to be optimistic about the potential discovery of supersymmetry at the LHC. One particular avenue for discovery is to investigate gluino-pair production for searches of the light stop squark [53].

\subsection{Light Stops}

The stop is special because it is the superpartner of the top quark, the heaviest of the Standard Model quarks. The dependence of the squark mass on the large mass of the top quark can render the mass of the stop much lighter than the other squarks. Furthermore, the off-diagonal terms in the squared-mass matrix are also dependent on the top mass and are much larger than the other squarks [65].

A stop squark with a mass that is less than that of the top quark is an attractive possibility for models of electroweak baryogenesis [66], a theory introduced in attempt to explain the matter-antimattcr asymmetry in the universe. Starting from initial conditions in which the asymmetry was zero, three conditions are required for baryogenesis in the early universe [67]:

- baryon number violation, 
- charge conjugation (C) and CP (combination of charge and parity (P)) violation,

- departure from thermal equilibrium (the baryon asymmetry generating processes must be out of thermal equilibrium).

The first statement requires there to be more processes in which baryons are produced then antibaryons and if CP is not violated, any existing process which creates an excess of baryons will have complimentary processes which produce excess antibaryons. The last criterion is necessary because a particle in thermal equilibrium would be destroyed and recreated through pair annihilation, washing out the asymmetry.

Although CP violation occurs in the Standard Model through a complex phase in the quark mixing matrix, there is not enough to account for the asymmetry seen in the universe [66]. The amount of CP-violation can be increased in the context of the MSSM which introduces new CP-violating interactions [66]. Furthermore, the third criteria may be satisfied in strong first order electroweak phase transitions [66], occurring at an energy scale at which the electroweak symmetry is broken. Within the MSSM, the conditions required to satisfy the special conditions of the phase transition are a light Higgs $(h)$ and a stop squark with a mass less than that of the top quark [68-71].

Previous searches for the stop provide lower mass limits, illustrated in Figure 4.2. Searches for stop-pair production were performed with LEP ( $\mathrm{e}^{ \pm}$collider) [72-74] and with the Tevatron (proton-antiproton collider) [75, 76]. The majority of the searches investigated the decay channel $\tilde{t} \rightarrow c \tilde{\chi}_{1}^{0}$; however, if the mass difference between the stop and the neutralino is small $(\Delta M<6 \mathrm{GeV})$, this channel is not available and the dominant decay channel becomes $\tilde{t} \rightarrow u \tilde{\chi}_{1}^{0}$. In this scenario, 


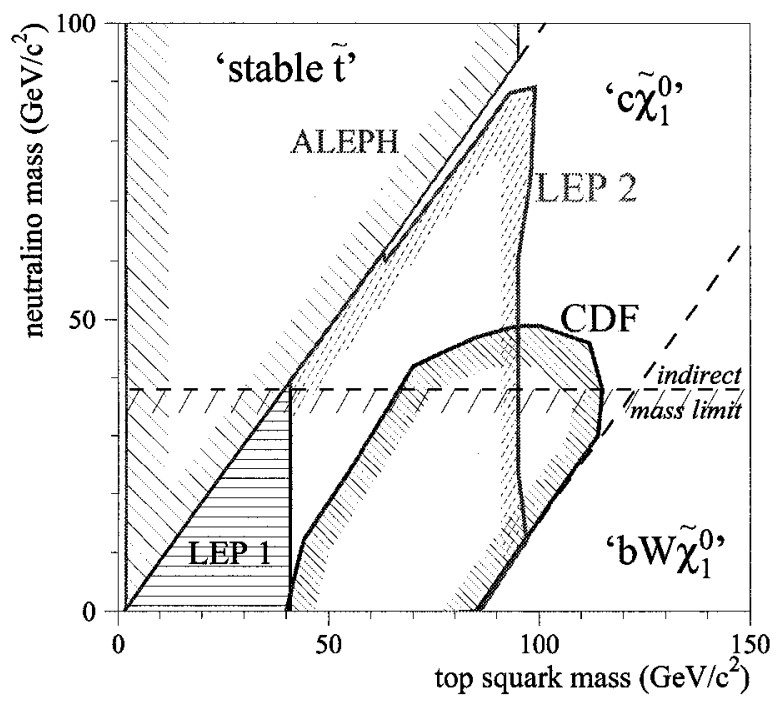

Figure 4.2: Stop mass limits. The shaded regions are excluded.

Figure taken from [77].

the stop can be considered a stable particle [72]. Events in excess of the Standard Model were not observed, leading to the mass limits in the $\left(\tilde{t}_{1}, \tilde{\chi}_{1}^{0}\right)$ plane.

With the knowledge of previous searches, the authors of [53] define an MSSM benchmark point called LST1 with a stop mass of $m_{\tilde{t}_{1}}=150 \mathrm{GeV}$ and investigate possible discovery with a generic LHC detector. These analysis techniques are adopted for searches with the ATLAS detector.

\subsubsection{LST1}

The presence of a light stop as required for electroweak baryogenesis, and consistency with the cold dark matter density described in Section 1.1 impose constraints on the parameters of supersymmetry. The input spectrum of particle parameters for the LST1 benchmark point is defined in [53] and illustrated in Figure 4.3. The sparticle masses are calculated in terms of the soft supersymmetry-breaking mass 


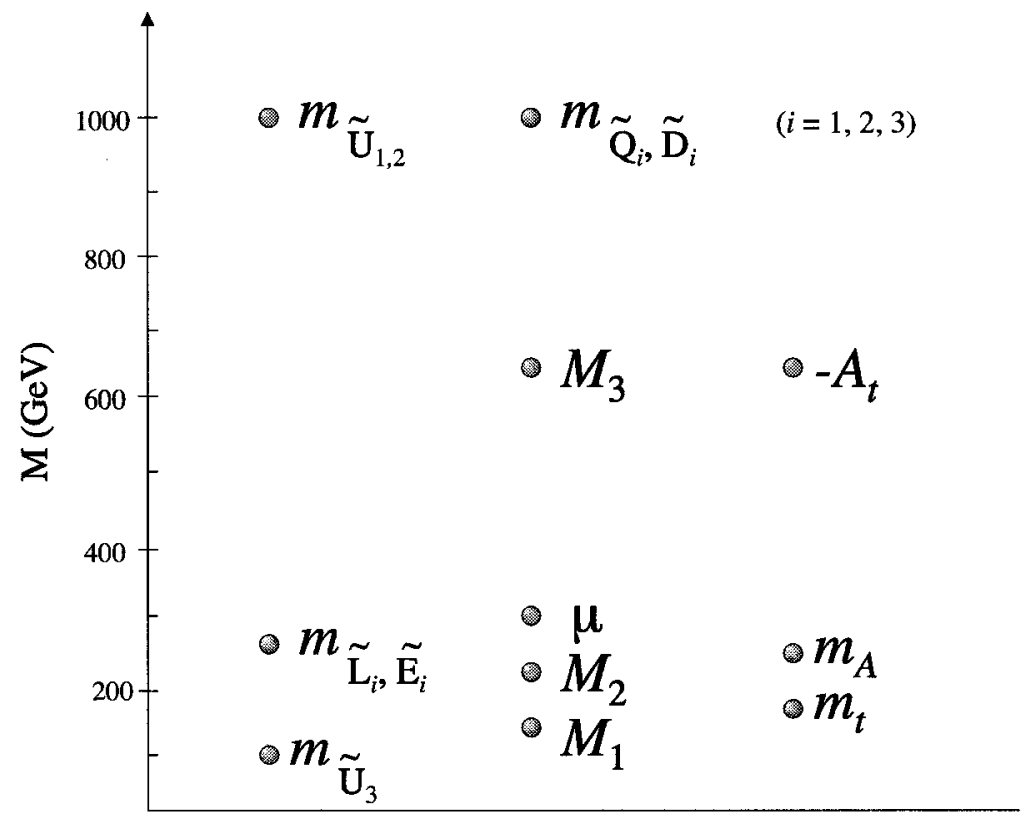

Figure 4.3: LST1 input spectrum [53]. In addition, $\tan \beta=7$.

parameters for the binos $\left(M_{1}\right)$, winos $\left(M_{2}\right)$, gauginos $\left(M_{3}\right)$, the left- and right-handed sleptons $\left(M_{\tilde{L}_{i}}, M_{\tilde{E}_{i}} ; i=1,2,3\right)$, the left- and right-handed squarks $\left(M_{\tilde{Q}_{i}}, M_{\tilde{U}_{i}}, M_{\tilde{D}_{i}}\right.$; $i=1,2,3)$, as well as the trilinear couplings $\left(A_{t}\right)$, the mass of the neutral Higgs $\left(m_{A}\right)$ and $\tan \beta$ of Equation (4.1). In addition, the sparticle masses are dependent on the supersymmetry conserving Higgsino mass parameter, $\mu$.

In order to limit the variation in parameters, all the sleptons were given a mass of $250 \mathrm{GeV}$ while all the squarks, apart from $\tilde{t}_{1}$, were given a mass of $1 \mathrm{TeV}$. It is assumed that the stop mixing occurs such that $\tilde{t}_{1} \sim \tilde{t}_{R}$ to preserve the agreement with the precision LEP data [66]. The values of $M_{1}, M_{2}$ and $\mu$ are set to $(110,220$, 300) $\mathrm{GeV}$ respectively as required for electroweak baryogenesis [70] while the gluino mass $\left(M_{3}\right)$ is set to $660 \mathrm{GeV}$. A value of $\tan \beta=7$ is used because CP-violating sources for the generation of baryon number are suppressed for values of $\tan \beta=10$ and higher. 


\begin{tabular}{c|c||c|c||c|c||c|c} 
Particle & Mass & Particle & Mass & Particle & Mass & Particle & Mass \\
\hline \hline$\tilde{\chi}_{1}^{0}$ & 104.680 & $\tilde{\chi}_{2}^{0}$ & 190.778 & $\tilde{\chi}_{3}^{0}$ & 306.161 & $\tilde{\chi}_{4}^{0}$ & 340.702 \\
\hline$\tilde{\chi}_{1}^{ \pm}$ & 189.083 & $\tilde{\chi}_{2}^{ \pm}$ & 339.866 & $\tilde{\tau}_{1}$ & 246.985 & $\tilde{\tau}_{2}$ & 260.757 \\
\hline$\tilde{t}_{1}$ & 148.764 & $\tilde{t}_{2}$ & 1018.853 & $\tilde{b}_{1}$ & 996.658 & $\tilde{b}_{2}$ & 1005.328 \\
\hline$h$ & 113.960 & $H$ & 251.612 & $H^{ \pm}$ & 262.179 & $\tilde{g}$ & 660.00
\end{tabular}

Table 4.3: Sparticle mass spectrum for the LST1 benchmark. All masses in $\mathrm{GeV}$.

Because the stop mass depends on the values of the right-handed squark mass parameter, the trilinear coupling $\left(A_{t}\right)$ and $\tan \beta$, their values must be adjusted for the fixed values given above. The non-zero value of $-A_{t}=670 \mathrm{GeV}$ simultaneously avoids constraints on the mass of the Higgs as well avoiding a decrease in the strength of the electroweak phase transition [70].

Because the sleptons are given a mass of $m_{\tilde{L}, \tilde{E}}=250 \mathrm{GeV}$, the mass of the neutral Higgs, A, is set to $m_{A}=250 \mathrm{GeV}$ in order to agree with the WMAP data [19] [20]. All the squark masses are set to $1 \mathrm{TeV}$ except for $m_{\tilde{U}_{3}}=100 \mathrm{GeV}$, maximizing the branching ratio of $\tilde{g} \rightarrow t \tilde{t}_{1}(100 \%)$ and suppressing background from interactions such as $\tilde{b} \rightarrow t \tilde{\chi}$. The resulting mass spectrum for the supersymmetric particles is given in Table 4.3, as calculated by ISAJET V7.64 [78]. Note that the stop has obtained a mass of $m_{\tilde{t}_{1}}=148.764 \mathrm{GeV}$ while the gluino has obtained a mass of $m_{\tilde{g}}=660.0 \mathrm{GeV}$. The lightest neutralino obtains a mass of $m_{\tilde{\chi}_{1}^{0}}=104.68 \mathrm{GeV}$, satisfying the bound $m_{\tilde{t}_{1}}-m_{\tilde{\chi}_{1}^{0}}<m_{W}$ enabling the decay $\tilde{t}_{1} \rightarrow c \tilde{\chi}_{1}^{0}$, which is assumed to be $100 \%$. 


\begin{tabular}{c||c|c|c|c|c|c|c}
$m_{\tilde{g}}(\mathrm{GeV})$ & 400 & 500 & 600 & 700 & 800 & 900 & 1000 \\
\hline \hline$\sigma(\tilde{g} \tilde{g})(\mathrm{pb})$ & 113 & 31.6 & 10.4 & 3.84 & 1.56 & 0.68 & 0.31
\end{tabular}

Table 4.4: NLO cross-section for gluino-pair production at the LHC.

\subsubsection{Light Stop Signature at the LHC}

In the LST1 scenario, gluinos will be pair-produced in abundance at the LHC. The next-to-leading order (NLO) cross-section for gluino-pair production is given as a function of the gluino mass in Table 4.4 [53]. If the gluino mass, $m_{\tilde{g}}$, satisfies $m_{\tilde{g}}>$ $m_{t}+m_{\tilde{t}_{1}}$, the gluino will decay into stops with a large branching ratio. Since the gluino is its own antiparticle (it is a Majorana fermion), the gluino will decay into $t \tilde{t}_{1}^{*}$ or $\bar{t}_{1}$ with equal probabilities. The decays of the pair-produced gluinos lead to

$$
\tilde{g} \tilde{g} \rightarrow t \bar{t} \tilde{t}_{1} \tilde{t}_{1}^{*}, t t \tilde{t}_{1}^{*} \tilde{t}_{1}^{*}, \tilde{t t \tilde{t}_{1}} \tilde{t}_{1}
$$

which provides same-sign (SS) top quarks in half of the decays. The Standard Model background consists mainly of $t \bar{t}$ events where one of the leptons stems from the decay of the $W^{ \pm}$and the other lepton comes from the semileptonic decay of a $b$-quark.

The decay chain is illustrated in Figure 4.4 for half of the gluino pair. For the events with same-sign top quarks, events are selected in which the $W^{ \pm}$decays leptonically (excluding $W^{ \pm} \rightarrow \tau \nu_{\tau}$ ) and the stop decays into a $c$-quark and the lightest supersymmetric particle, $\tilde{\chi}_{1}^{0}$. The decay to $c \tilde{\chi}_{1}^{0}$ must proceed through loop effects, but is the dominant decay if $m_{\tilde{t}_{1}}<m_{\tilde{\chi}_{1}^{ \pm}}+m_{b}$ and $m_{\tilde{t}_{1}}-m_{\tilde{\chi}_{1}^{0}}<m_{W^{ \pm}}$. The $b$ - and $c$ quarks appear as 'jets' (collimated showers of particles resulting from the production of quarks and gluons) in the detector, while the neutrino and the neutralino will escape undetected, giving rise to missing transverse energy $\left(\mathbb{F}_{T}\right)$. Remaining is a 


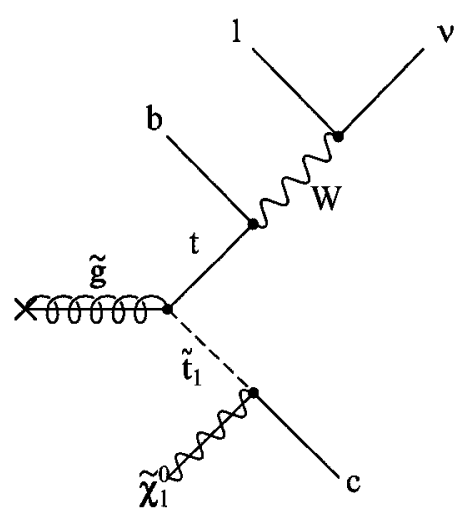

Figure 4.4: Decay chain for half of the gluino-pair produced in proton-proton collisions.

signature with great discriminating power against Standard Model backgrounds:

$$
2 b+2 \mathrm{SS} \text { leptons }+ \text { jets }+\mathbb{E}_{T} .
$$

This signature will be utilized in Section 4.2.5.

\subsubsection{Monte Carlo Production}

As mentioned in Section 2.2.5, the physics of the proton-proton collisions of the LHC for Monte Carlo studies are performed using event generators. For this study, the parton showers and hadronization were calculated using HERWIG 6.510 [79-82]. The total supersymmetric production cross-section for the LST1 benchmark point was calculated to be $161 \mathrm{pb}$ and the cross-section for two-gluino events was calculated to be $5 \mathrm{pb}$. The detector response to the input events were simulated using the ATLFAST detector simulation described in Section 2.2.5 and Appendix B.

The Standard Model backgrounds were generated by the ATLAS SUSY working group [83]. The Standard Model backgrounds that have been studied are $t \bar{t}, \mathrm{QCD}$, $W^{ \pm}+$jets and $Z^{0}+$ jets. The backgrounds were also simulated using ATLFAST. 


\subsubsection{Inclusive Searches}

\subsubsection{Effective Mass}

A first variable to investigate in all SUSY models is the effective mass [84], defined as

$$
M_{e f f}=\not \notin_{T}+\sum p_{T}^{j e t}
$$

where the sum is over all of the jets. This is considered an 'inclusive' search because all production channels are considered in the analysis. The cuts used to isolate the signal (assuming lepton charge identification of 100\%) are:

- 2 leptons $(\mathrm{e}, \mu)$ and $\geq 4$ jets $(2 l, 4 \mathrm{jet})$,

- each lepton with $p_{T}>20 \mathrm{GeV}\left(l_{p_{T}}\right)$,

- each jet with $p_{T}>50 \mathrm{GeV}\left(\right.$ jet $\left._{p_{T}}\right)$,

- $\mathbb{E}_{T}>100 \mathrm{GeV}\left(\mathbb{E}_{T}\right)$,

- 2 same-sign leptons (SS),

resulting in a selection efficiency of $0.80 \%$ for the signal.

The distribution for effective mass for all LST1 events is shown in Figure 4.5 along with the Standard Model backgrounds for an integrated luminosity of $30 \mathrm{fb}^{-1}$. The hatched histogram is the total background. The $t \bar{t}$ events are the dominant background, as only three events from the $W+$ Njet background survived the cuts, while the $Z+$ Njet and QCD backgrounds did not survive (although limited statistics were available for QCD). There is clearly an excess of events at the high end of the distribution, indicating that SUSY would be easily discovered at the LHC in this light stop scenario. 


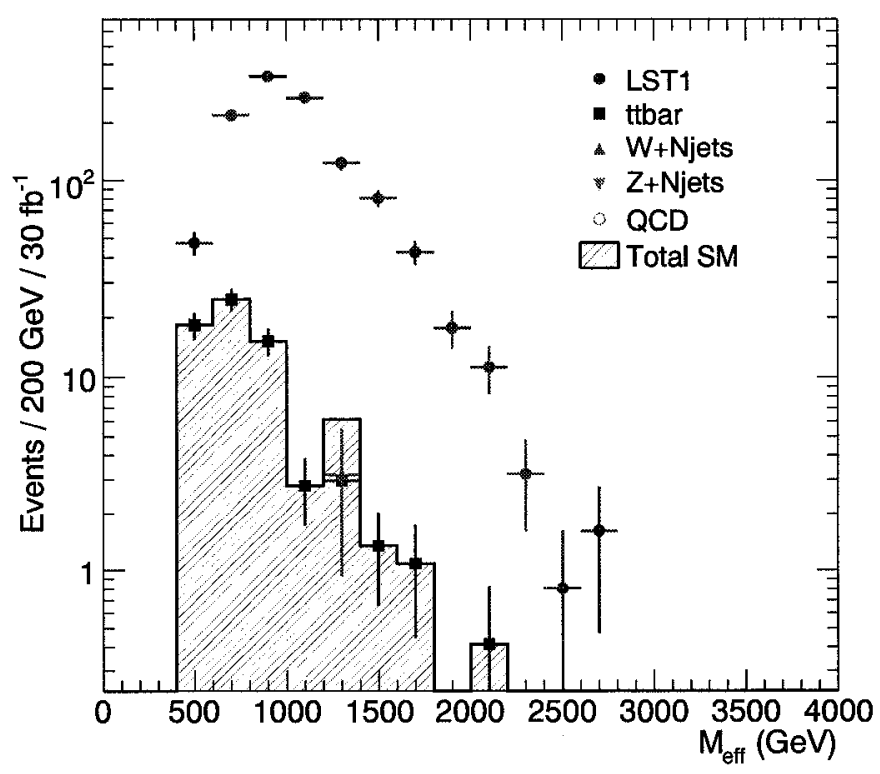

Figure 4.5: Effective mass for LST1 for an integrated luminosity of $30 \mathrm{fb}^{-1}$.

\subsubsection{Exclusive Searches}

\subsubsection{Significance}

In the exclusive searches, only the gluino-pair producing events will be isolated and analyzed. As mentioned in Section 4.2, the Majorana nature of the gluino leads to the signature of two same-sign leptons at the LHC, to which there is very little Standard Model background. The signal selection chosen for the exclusive studies is similar to those in Section 4.2, with two additional cuts defined in [53]:

- at least 2 jets that are $b$-tagged $(2 b)$,

- 2 top candidates with $M_{b l}<160.0 \mathrm{GeV}(2 t)$.

In ATLFAST, the $b$-jets are identified with an efficiency $60 \%$ [34]. 
The number of events surviving each cut is shown in Table 4.5 for both the signal and the backgrounds. To study the signal, a filtering algorithm was utilized on the event generator to select only the events in which two gluinos were produced. The SUSY background consists of the excess events which pass the cut criteria when the gluino filter is not applied. Of the SM background, only the $t \bar{t}$ events have nonzero results after the cut on two top candidates. The non-integer value of the $t \bar{t}$ background is due to scaling down to $30 \mathrm{fb}^{-1}$.

Assuming a Poisson distribution for the number of background events, a $90 \%$ confidence level on the upper limit can be placed on the number of $t \bar{t}$ events at $<4.62$ and the number of SUSY background events $<10.53$.

The cut flow was also studied for scenarios with different gluino masses. The results are shown in Table 4.6 and the significance is shown in Figure 4.6. The significance is defined as $S / \sqrt{B}$ where $S$ is the number of signal events and $B$ is the sum of the Standard Model and SUSY background events.

The significance is shown to decrease as the mass of the gluino increases. The significance with the jet $p_{T}$ cut changed to $20 \mathrm{GeV}$ for $\mathrm{m}_{\tilde{g}}=660 \mathrm{GeV}$ is also shown for purposes discussed in the next section. With the new jet $\mathrm{p}_{T}$ cut value, the significance increases slightly.

\subsubsection{Invariant Masses}

If the inclusive studies give positive results for physics beyond the Standard Model and the signal selection helps isolate the decay chain, it is important to measure the properties of the new particle states to confirm that they are part of the light stop scenario. The usual method of studying the masses is using the invariant mass 


\begin{tabular}{c||c|c|c|c|c|c|c|c}
\multicolumn{1}{c||}{} & \multicolumn{10}{c}{ Cut } \\
\cline { 2 - 9 } \multicolumn{2}{c||}{} & $2 l, 4$ jet & $l_{p_{T}}$ & jet $_{p_{T}}$ & $2 b$ & $E_{T}$ & $2 t$ & SS \\
\hline \hline \multirow{2}{*}{ Signal } & $\tilde{g} \tilde{g}$ & 5114 & 4440 & 2783 & 647 & 520 & 382 & 189 \\
\hline \multirow{3}{*}{ Background } & SUSY & 742 & 668 & 240 & 36 & 29 & 11 & 6 \\
& $t \bar{t}$ & 68739 & 56758 & 11064 & 2587 & 1209 & 1101 & 1.5 \\
& $W^{ \pm}+$jets & 150 & 29 & 15 & 2 & 2 & 0 & 0 \\
& $Z^{0}+$ jets & 2 & 1 & 0 & 0 & 0 & 0 & 0
\end{tabular}

Table 4.5: Cut flow for reconstructed signal and both SUSY and Standard Model backgrounds for an integrated luminosity of $30 \mathrm{fb}^{-1}$. QCD backgrounds are found to be negligible. The cut names are defined in the text.

\begin{tabular}{c|c||c|c|c|c|c|c|c}
\multicolumn{2}{c||}{} & \multicolumn{8}{c}{ Cut } \\
\hline$m_{\tilde{g}}(\mathrm{GeV})$ & & $2 l, 4$ jet & $l_{p_{T}}$ & jet $_{p_{T}}$ & $2 b$ & $E_{T}$ & $2 t$ & SS \\
\hline \hline \multirow{2}{*}{400} & Signal & 63160 & 52531 & 14585 & 3425 & 2224 & 1918 & 926 \\
& SUSY Bg. & 993 & 866 & 268 & 42 & 35 & 23 & 11 \\
\hline \multirow{2}{*}{660} & Signal & 5114 & 4440 & 2783 & 647 & 520 & 382 & 189 \\
& SUSY Bg. & 742 & 668 & 240 & 36 & 29 & 11 & 6 \\
\hline \multirow{2}{*}{900} & Signal & 699 & 642 & 446 & 112 & 97 & 71 & 37 \\
& SUSY Bg. & 801 & 718 & 251 & 25 & 21 & 6 & 5
\end{tabular}

Table 4.6: Cut flow for reconstructed signal and SUSY background (Bg.) with varying gluino masses for an integrated luminosity of $30 \mathrm{fb}^{-1}$. The cut names are defined in the text. 


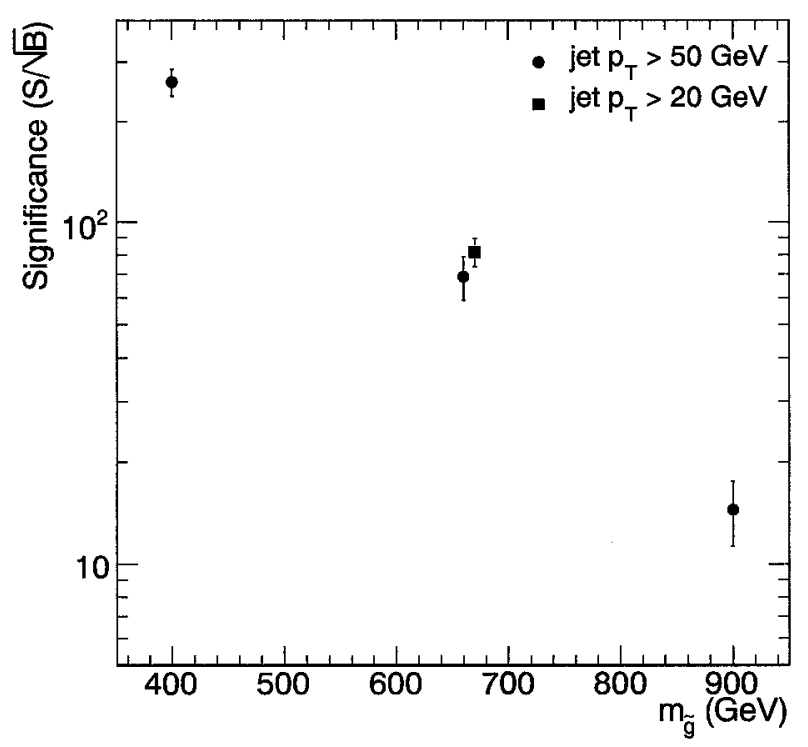

Figure 4.6: Significance $(S / \sqrt{B})$ for different gluino masses for an integrated luminosity of $30 \mathrm{fb}^{-1}$.

distributions of the particles involved in the decay chain [84], where invariant mass is defined in terms of the square of the sum of the 4-momentum, $\left(p^{\mu}\right)$, of the particles involved:

$$
M^{2}=\left(\sum_{i} p_{i}^{\mu}\right)^{2} .
$$

The endpoints, $M^{\max }$, are defined in terms of the masses of the particles involved in the chain.

In the particular decay chain under study, there are 4 possible invariant mass contributions; $M_{b l}, M_{b c}, M_{l c}$ and $M_{b l c}$. Out of these four, the first only relates the mass of the top quark and the mass of the $W^{ \pm}$. In addition, $M_{l c}^{\max }$ and $M_{b c}^{\max }$ are not independent:

$$
\left(M_{l c}^{\max }\right)^{2}=\left(M_{b c}^{\max }\right)^{2} \frac{m_{t}^{2}}{m_{W}^{2}}
$$

Thus, the system cannot be solved uniquely and only relations between the neutralino 


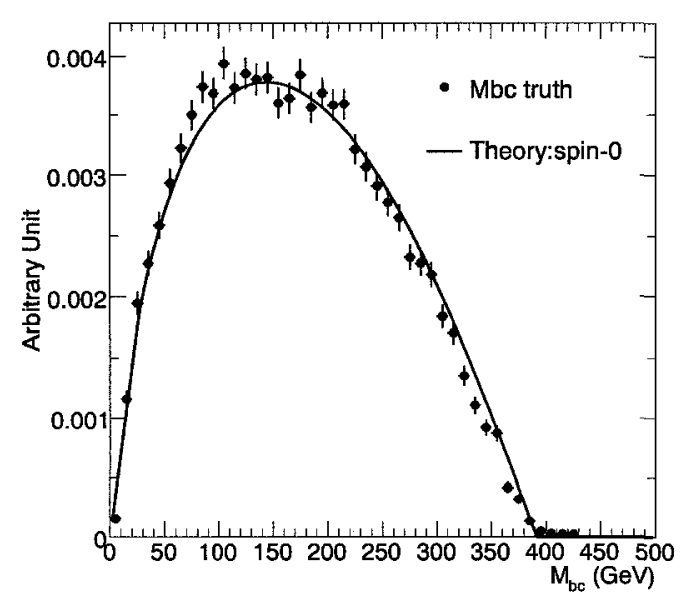

(a) $M_{b c}$

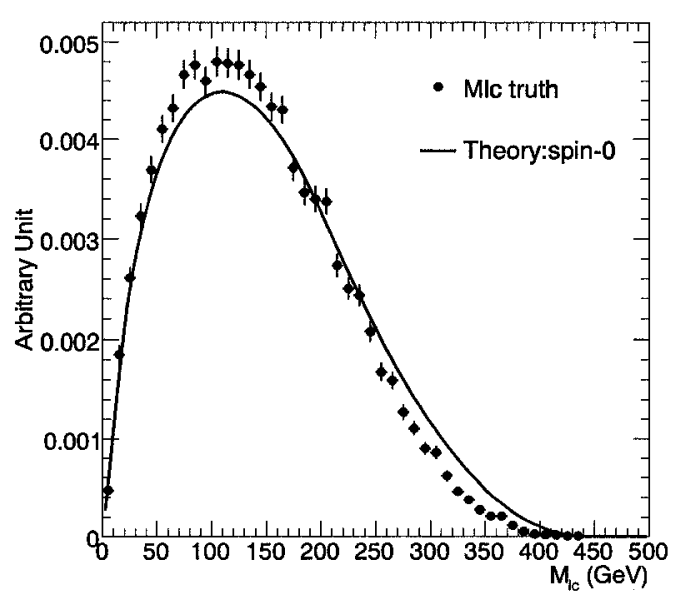

(b) $M_{l c}$

Figure 4.7: Invariant mass distributions for (a) $M_{b c}$ and (b) $M_{l c}$. Both distributions are at the parton level and cuts have not been applied. Overlaid is the theoretical distribution where $a=0.991$ and $M_{b c}^{\max }=392 \mathrm{GeV}$.

and stop masses can be determined.

The authors of [53] have extended the endpoint method by deriving analytic formulas for the invariant mass distributions (not including spin correlations). The analytic formulas, given in Appendix $\mathrm{C}$, allow for the fit of two parameters; $a$ and the endpoint $M_{b c}^{\max }$, where

$$
\begin{gathered}
a \equiv \frac{m_{2}^{2}}{m_{1}^{2}} \\
m_{1}^{2}=m_{\tilde{g}}^{2}-m_{t}^{2}-m_{\tilde{t}}^{2}, \quad m_{2}^{4}=m_{1}^{4}-4 m_{t}^{2} m_{\tilde{t}}^{2} \\
\left(M_{b c}^{\max }\right)^{2}=\frac{\left(m_{t}^{2}-m_{W}^{2}\right)}{m_{t}^{2}} \frac{\left(m_{\tilde{t}_{1}}^{2}-m_{\tilde{\chi}_{1}^{0}}^{2}\right)\left(m_{1}^{2}+m_{2}^{2}\right)}{2 m_{\tilde{t}_{1}}^{2}} .
\end{gathered}
$$

The nominal values for these parameters in the LST1 benchmark are $a=0.991$ and $M_{b c}^{\max }=392 \mathrm{GeV}$. 
The invariant mass distributions are shown in Figure 4.7 with no cuts applied. Overlaid are the theoretical formulas. The results are obtained using the parton information, meaning neither the detector simulation nor the reconstruction algorithms have been implemented on the generated events (referred to as Monte Carlo 'truth' information). Note that these distributions assume no spin correlations [53] between the particles. The spin correlations tend to shift the peaks of the invariant mass distributions to the higher end of the spectrum. For these reasons, all events were generated without spin correlations. The differences between the parton level results and the theoretical curve for $M_{l c}$ near the peak and in the high invariant mass tail are mainly a result of final state radiation (FSR).

At the reconstruction level, the invariant masses were only exposed to the kinematic cuts proposed in [53], approximately $40 \mathrm{GeV}$ above the nominal values of the invariant mass endpoints:

- $M_{b c}<430 \mathrm{GeV}$

- $M_{l c}<480 \mathrm{GeV}$,

- $M_{b l c}<505 \mathrm{GeV}$.

The cuts were defined in [53] to demand consistency with the theoretical endpoints calculated using Equation (4.10) and Equation (4.7).

Because $c$-jets are not identified in the ATLAS detector, they were selected as the two jets with the largest $p_{T}$ that were not $b$-tagged and they were paired to the top candidates by minimizing the angular separation $\left(\Delta R=\sqrt{(\Delta \eta)^{2}+(\Delta \phi)^{2}}\right)$. With this method, approximately $55 \%$ of the pairs were found to be correct.

The fit values obtained for the $M_{b c}$ and $M_{l c}$ distributions are labelled 'Recon- 


\begin{tabular}{c|c||c|c} 
Method & Distribution & $a$ & $M_{b c}^{\max }(\mathrm{GeV})$ \\
\hline \hline Reconstruction & $M_{b c}$ & $0.69 \pm 0.01$ & $443 \pm 7$ \\
& $M_{l c}$ & $0.77 \pm 0.07$ & $390 \pm 12$ \\
\hline jet $p_{T}>20 \mathrm{GeV}$ & $M_{b c}$ & $0.86 \pm 0.04$ & $442 \pm 6$ \\
& $M_{l c}$ & $0.83 \pm 0.05$ & $385 \pm 12$ \\
\hline \multirow{2}{*}{ Correction Function } & $M_{b c}$ & $0.92 \pm 0.07$ & $408 \pm 8$ \\
& $M_{l c}$ & $0.97 \pm 0.03$ & $381 \pm 13$
\end{tabular}

Table 4.7: Fit results for the different invariant mass distributions. The nominal values are $a=0.991$ and $M_{b c}^{\max }=392 \mathrm{GeV}$.

struction' in Table 4.7. The values for $a, 0.69$ and 0.77 , calculated from $M_{b c}$ and $M_{l c}$ respectively, are affected by the shape of the distribution and are inconsistent with the predicted values.

The effects of the cuts are illustrated in Figure 4.8 for $M_{b c}$. The invariant mass using truth objects are shown Figure 4.8a, with and without the applied cuts, while Figure $4.8 \mathrm{~b}$ shows the comparison of the invariant masses using reconstructed objects to the invariant masses using truth objects with cuts applied to both. It was determined that the jet $p_{T}$ cut had the largest effect, shifting the peak of the distribution to the higher end of the spectrum. The discrepancies in the high invariant mass tails in Figure $4.8 \mathrm{~b}$ are caused by the pairing impurities mentioned above.

Two approaches were explored to correct the distorted distributions. In the first method, the jet $p_{T}$ cut was lowered to $20 \mathrm{GeV}$, since this was determined to be the major effect. The results of the fits are provided in Table 4.7. The distribution shapes are somewhat restored and because the selection criteria is altered, the significance is expected to change. The slight increase in the significance of the signal is shown 


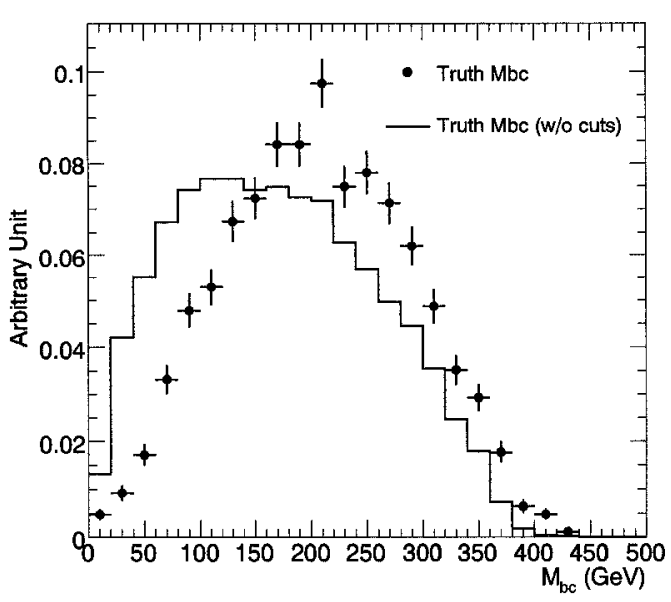

(a)

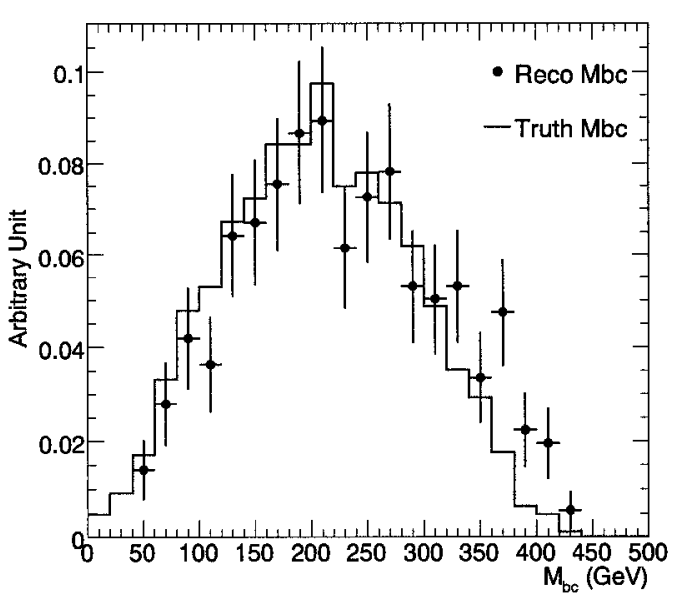

(b)

Figure 4.8: Invariant mass distributions for $M_{b c}$ normalized to unit area. (a) Comparison between truth results with and without cuts. (b) Comparison between truth and reconstructed results.

in Figure 4.6.

The second method applied an acceptance correction function to the invariant mass distributions. The function was calculated as the ratio of all the truth events to the truth events which passed the selection criteria (the ratio of distributions in Figure 4.8a). The correction functions for $M_{b c}$ and $M_{l c}$ can be seen in Figure 4.9a along with the corrected invariant mass distributions. Figures $4.9 \mathrm{~b}$ and $4.9 \mathrm{c}$ show that the distributions are restored once the correction functions are applied. The results for all of the fit values are given in Table 4.7. In all three methods, the fit values are obtained using an independent fit to each of the individual invariant mass distributions. Simultaneous fits to $M_{b c}$ and $M_{l c}$ were not attempted due to the nontrivial statistical correlations between the distributions. The fit results indicate that the correction function method is the most promising in restoring the shapes of the 
distributions.

\subsubsection{Systematic Uncertainties}

To estimate the systematic uncertainties, changes were made in the parton distribution function (PDF), the mass of the top quark, the $b$-tagging efficiency, the lepton charge identification efficiency and the jet energy scale uncertainty (JES). Events were generated for the different PDFs, the different masses of the top quark and with variations in the $b$-tagging efficiency, while the lepton charge efficiency and jet energy scale were modified at the analysis level. The changes included:

- PDF: CTEQ6L $\rightarrow$ CTEQ6M [85],

- $m_{t}: 175 \mathrm{GeV} \rightarrow(172,178) \mathrm{GeV}[6]$

- JES: $\pm 5 \%[86]$

- $b$-tag Efficiency: $60 \% \rightarrow 50 \%, 70 \%$,

- Lep. Charge Efficiency: $100 \% \rightarrow 80 \%$ [86].

The results of the number of events passing the selection criteria of Section 4.2.5 are given in Table 4.8. The lower bound of the lepton charge efficiency was chosen as a conservative estimation and at $90 \%$ efficiency, the effect becomes negligeable. The systematic error is calculated as the difference between the number of events shown in Table 4.8 to the number of events passing with the default settings (Table 4.5). The systematic and statistical errors are approximately equal for the PDF and $m_{t}=178 \mathrm{GeV}$, while the statistical errors dominate the total error for the lepton charge efficiency and the jet energy scale. For this reason, the quoted 


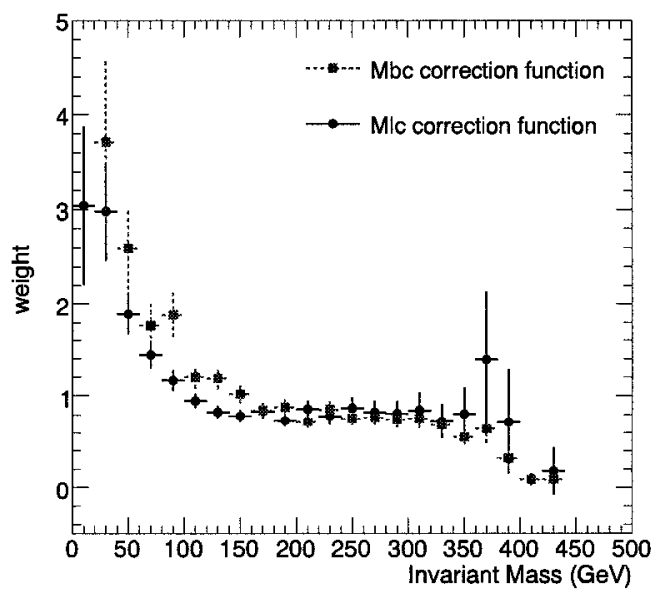

(a) Acceptance Correction Functions

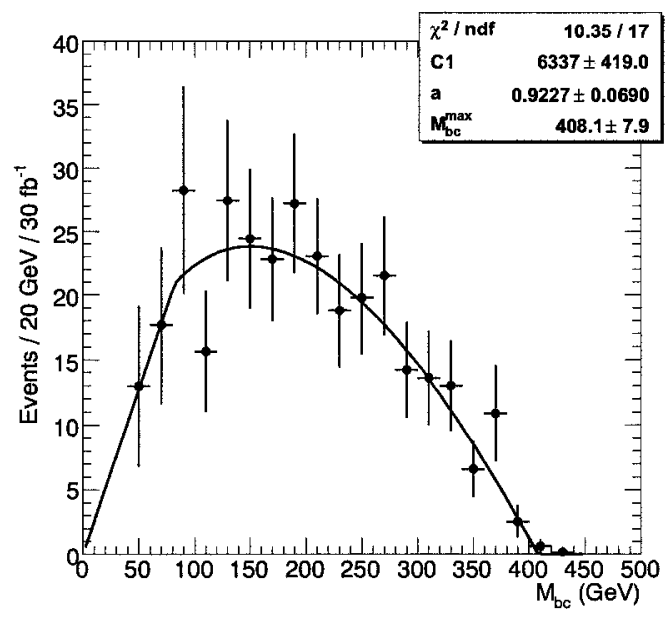

(b) Corrected $M_{b c}$

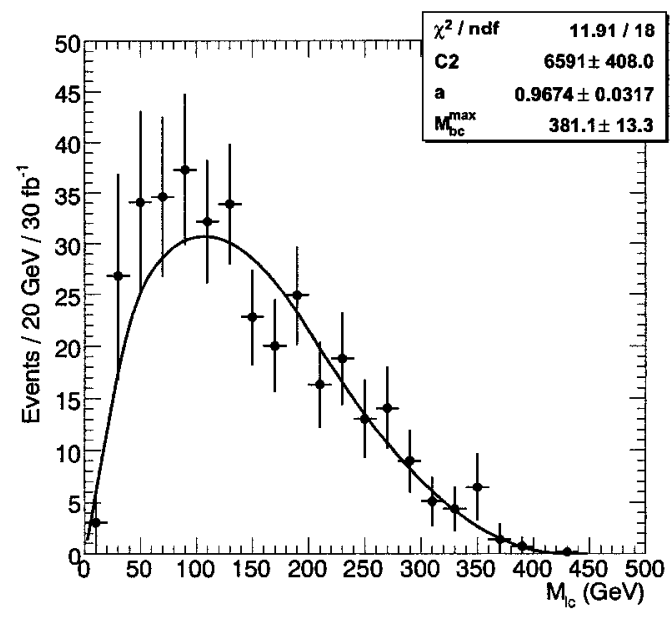

(c) Corrected $M_{l c}$

Figure 4.9: (a) The acceptance correction functions and resulting corrected distributions (with fits) for the invariant mass distributions (b) $M_{b c}$ and (c) $M_{l c}$. The curves are the resulting fits to the data using (b) Equation (C.2) and (c) Equation (C.3). 


\begin{tabular}{c|c||c|c|c}
\multicolumn{2}{c||}{} & N & Stat (\%) & Syst (\%) \\
\hline \hline PDF & CTEQ6M & 204 & 7 & 8 \\
\hline \multirow{2}{*}{$m_{t}$} & $172 \mathrm{GeV}$ & 189 & 7 & 0 \\
& $178 \mathrm{GeV}$ & 204 & 7 & 8 \\
\hline \multirow{2}{*}{ JES } & $-5 \%$ & 183 & 7 & 3 \\
& $+5 \%$ & 196 & 7 & 4 \\
\hline \multirow{2}{*}{$b$-tag Eff. } & $50 \%$ & 136 & 9 & 28 \\
& $70 \%$ & 283 & 6 & 50 \\
\hline Lep Charge Eff. & $80 \%$ & 182 & 7 & 4 \\
\hline \hline Total & & & & 52
\end{tabular}

Table 4.8: Statistical and systematic uncertainties of selection criteria of the light stop signal for $30 \mathrm{fb}^{-1}$ of data. The errors shown are the fractional errors. The total systematic uncertainty is obtained by addition of individual uncertainties in quadrature (see text for details).

systematic uncertainties are considered conservative estimates. Along with the PDF and lepton charge uncertainties, the total systematic uncertainty is calculated using the larger uncertainty in the top mass $\left(8 \%\right.$ for $\left.m_{t}=178 \mathrm{GeV}\right)$, jet energy scale $(4 \%$ uncertainty for JES $=+5 \%)$ and $b$-tagging efficiency ( $50 \%$ uncertainty for $b$-tag $=$ $70 \%$ ). The total uncertainty of $52 \%$ is obtained by adding the above uncertainties in quadrature. The dominant uncertainty comes from the $b$-tagging efficiency.

The systematic uncertainties for the invariant mass fit parameters were also calculated and are given in Table 4.9. The distributions were calculated using the correction function provided in Figure 4.9a. The systematic uncertainties are calculated 


\begin{tabular}{|c|c|c|c|c|c|}
\hline \multirow{2}{*}{ Systematic } & \multirow{2}{*}{ Distribution } & \multicolumn{2}{|c|}{$\delta a(\%)$} & \multicolumn{2}{|c|}{$\delta M_{b c}^{\max }(\%)$} \\
\hline & & Stat. & Syst. & Stat. & Syst. \\
\hline \multirow{2}{*}{$\mathrm{PDF}$} & $M_{b c}$ & 6 & 4 & 2 & 6 \\
\hline & $M_{l c}$ & 4 & 4 & 3 & 5 \\
\hline$m_{t}$ & $M_{b c}$ & 4 & 5 & 1 & 3 \\
\hline$(172 \mathrm{GeV})$ & $M_{l c}$ & 4 & 3 & 4 & 0.5 \\
\hline \multirow{2}{*}{$\begin{array}{c}m_{t} \\
(178 \mathrm{GeV})\end{array}$} & $M_{b c}$ & 6 & 3 & 5 & 1 \\
\hline & $M_{l c}$ & 3 & 0.2 & 5 & 1 \\
\hline \multirow{2}{*}{$\begin{array}{c}\text { JES } \\
(-5.0 \%)\end{array}$} & $M_{b c}$ & 6 & 2 & 2 & 0 \\
\hline & $M_{l c}$ & 3 & 0.2 & 3 & 1 \\
\hline \multirow{2}{*}{$\begin{array}{c}\text { JES } \\
(+5.0 \%)\end{array}$} & $M_{b c}$ & 8 & 5 & 1 & 1 \\
\hline & $M_{l c}$ & 4 & 1 & 3 & 3 \\
\hline \multirow{2}{*}{$\begin{array}{c}b \text {-tag Eff. } \\
(50 \%)\end{array}$} & $M_{b c}$ & 8 & 20 & 2 & 8 \\
\hline & $M_{l c}$ & 9 & 13 & 4 & 5 \\
\hline \multirow{2}{*}{$\begin{array}{c}b \text {-tag Eff. } \\
(70 \%)\end{array}$} & $M_{b c}$ & 5 & 5 & 1 & 8 \\
\hline & $M_{l c}$ & 3 & 0.4 & 3 & 3 \\
\hline \multirow{2}{*}{ Lep Charge Eff. } & $M_{b c}$ & 8 & 3 & 1 & 1 \\
\hline & $M_{l c}$ & 4 & 2 & 4 & 2 \\
\hline \multirow{2}{*}{ Total } & $M_{b c}$ & & 21 & & 11 \\
\hline & $M_{l c}$ & & 14 & & 8 \\
\hline
\end{tabular}

Table 4.9: Statistical and systematic uncertainties of invariant mass fit parameters for $30 \mathrm{fb}^{-1}$ of data. The total systematic uncertainty is obtained by addition of individual uncertainties in quadrature (see text for details). 
as the difference in the fit parameter values with the values provided in Table 4.7. Once again, independent fits for the individual invariant mass distributions were used. Because the correction function is calculated with the default values of PDF and the top mass, the uncertainty in the fit parameter values for the different PDF and top masses contains a component due to the correction method.

The systematic uncertainty dominates the statistical error for the value of $a$ only in the case of the $b$-tagging efficiency. The statistical errors for $M_{b c}^{\max }$ are larger or equal to the systematic uncertainties except for the variation in the PDF, the decrease in the top mass and the $b$-tagging efficiency of $50 \%$.

A conservative total systematic uncertainty is obtained by adding the larger of the top mass uncertainties, the larger of the JES uncertainties and the larger of the $b$-tagging efficiency in quadrature. The total systematic uncertainties for $\left(a, M_{b c}^{\max }\right)$ are $(21 \%, 11 \%)$ and $(14 \%, 8 \%)$, calculated using the $M_{b c}$ and $M_{l c}$ distributions, respectively.

\subsection{Summary}

The search for a light stop particle with the ATLAS detector is feasible. The Majorana nature of the gluino produces events with same-sign leptons in the final state which, proves to be a powerful discriminant over both Standard Model and SUSY backgrounds. Within the LST1 scenario, the effective mass is useful to identify excess events over the Standard Model. The significance is shown to decrease with increasing gluino mass, however it remains at an acceptable level even as the gluino mass reaches $900 \mathrm{GeV}$. 
The invariant mass distributions developed in [53] may provide useful information about the SUSY masses. Care must be taken when utilizing the invariant mass distributions, $M_{b c}$ and $M_{l c}$, since the jet $p_{T}$ cut was shown to influence the shape of the distribution.

The above Monte Carlo studies were performed on a parameterized version of the ATLAS detector to reproduce the results in [53]. Further study is required with the fully simulated version of the ATLAS detector in order to account for the real detector performance in terms of jet energy scale, $\mathbb{E}_{T}$, lepton fakes, etc., as well as to optimize the analysis techniques. For instance, multivariate techniques may be applied when trying to identify the correct pairs from the leptons, $b$ - and $c$-jets. Also, the kinematics cuts defined in Section 4.2.5.2 for the invariant masses should be altered so as not to rely on the SUSY masses which are unknown parameters. 


\section{Chapter 5}

\section{Summary}

The combination of large centre-of-mass energy proton collisions and the unprecedented design luminosity of the Large Hadron Collider will reveal many physics channels for investigation with the ATLAS detector, including both detailed studies of the Standard Model along with the direct search for new physics phenomena.

One important signature in many extensions of the Standard Model is missing energy. The forward calorimeter completes the hermetic design of the ATLAS calorimeter system and is responsible for the measurement of the missing transverse energy near the beampipe in the forward direction. In 2003 a beam test was performed on the FCal using beams of electrons and hadrons in the energy range of $(10-200) \mathrm{GeV}$. A Monte Carlo toolkit for the beam test has been implemented in the ATLAS software framework. Monte Carlo results for the electron sampling fractions of the FCal are shown to be independent of energy over the above range. In addition, the electron response of the detector in terms of linearity and energy resolution over the above energies are presented. The deviation from linearity for the Monte Carlo was found to be within $0.5 \%$, compared to $0.8 \%$ for data. The resolution 
was found to be dependent on the Geant4 physics list used; however the Monte Carlo continues to underestimate the resolution as compared to the beam test data.

The search for supersymmetry will be a major focus of the ATLAS collaboration. Analysis techniques are adopted for the ATLAS detector to search for a light superpartner of the top quark. A supersymmetric benchmark point called LST1 was defined in [53] and studied here with ATLFAST, the parameterized version of the ATLAS detector simulation. The same-sign di-lepton signature of gluino-pair production in LST1 proved to provide sufficient discriminating power against Standard Model background to allow for discovery with $30 \mathrm{fb}^{-1}$ of integrated luminosity, which corresponds to three years of running at low-luminosity (see Appendix A). Furthermore, it was demonstrated that mass information about the lightest neutralino and the stop can be obtained using the invariant mass distributions derived in [53]. The feasibility of this supersymmetry study as shown by the fast simulation is promising and indicates that more in-depth analysis should be performed with the full simulation of the ATLAS detector, in preparation for data. 


\section{Appendix A}

\section{Collider Physics Parameters}

Three important quantities in high energy particle collider experiments are the energy of the collisions, $E$, the luminosity of the colliding beams, $L$, and the cross-section of the process under study, $\sigma$.

The cross-sections are characteristic of the underlying physics interaction and are generally a function of energy. Higher energy collisions allow for the production of more massive particles in the interactions. The dimensions of cross-section are [length $\left.{ }^{2}\right]$ with the unit of 'barn' (b) introduced to deal with the short distance scales associated with nuclear and particle physics:

$$
\mathrm{b} \equiv 10^{-24} \mathrm{~cm}^{2}
$$

The reaction rate of the process is proportional to the cross-section,

$$
R=L \sigma,
$$

and the proportionality constant is called the luminosity. The dimension of luminosity 
are $\left[\right.$ length ${ }^{-2}$ time $\left.^{-1}\right]$. The luminosity is a characteristic of the beam:

$$
L=\frac{k f N^{2}}{4 \pi \sigma_{x} \sigma_{y}}
$$

where $k$ represents the number of bunches per beam (2808 for LHC), $f$ is the revolution frequency $(11.23 \mathrm{kHz}), N$ is the number of protons per bunch $\left(1.15 \times 10^{11}\right)$ and $\sigma_{x / y}$ is the size of the beam at the collision point $\left(\sigma_{x}=\sigma_{y}=16 \mu \mathrm{m}\right)$. These values result in the design luminosity of the $\mathrm{LHC}, L=10^{34} \mathrm{~cm}^{-2} \mathrm{~s}^{-1}$. During the early stages of running, before the design luminosity is reached, the LHC will run at a luminosity of $10^{31} \mathrm{~cm}^{-2} \mathrm{~s}^{-1}$, followed by three years of running at "low" luminosity, $10^{33} \mathrm{~cm}^{-2} \mathrm{~s}^{-1}$. One year of running at the LHC is approximately 100 days, amounting $10 \mathrm{fb}^{-1} / \mathrm{yr}$ of data during the low luminosity stage.

The number of events over a given time period is equal to the cross-section times the luminosity (integrated over time):

$$
N=\sigma \int L d t
$$

Data sizes are usually quoted in terms of the integrated luminosity, $\mathcal{L}=\int L d t$. As an example, $30 \mathrm{fb}^{-1}$ of data will be collected in approximately three years of low luminosity of running at the LHC. 


\section{Appendix B}

\section{ATLFAST Physics Objects}

The following is taken from the ATLFAST paper. Please see [34] for more details.

\section{B.1 Reconstruction of Physics Objects in ATL- FAST}

The reconstruction of physics object in ATLFAST relies heavily on the truth information made available from the event generators. There is no reconstruction layer that is based on the simulated detector information, except for the application of a simple seed cone algorithm for the cluster reconstruction in the calorimeters. The following sections describe how the physics objects are reconstructed in ATLFAST. 


\section{Clusters}

The clusters are the first objects to be reconstructed and are based on the map of deposited energies on which a simple cluster algorithm is performed. The cone algorithm uses a cone size of $\Delta \mathrm{R}=0.4$, where $\Delta \mathrm{R}=\sqrt{(\Delta \eta)^{2}+(\Delta \phi)^{2}}$. The cone is initiated by seed cells with energies greater than $1.5 \mathrm{GeV}$ and the cone algorithm is applied to seed cells in decreasing order of $p_{T}$. Any particular cell can only be associated with one cluster and in order for the cluster to be considered, the cluster energy must be greater than an energy threshold of $5 \mathrm{GeV}$. As other objects are reconstructed, clusters may get reclassified as electrons, photons, etc., in which case they are removed from the cluster container.

\section{Electrons}

The truth information for electrons is used to match to a cluster in the list described above. The default separation for a match of a truth electron to a cluster is $\Delta R=0.15$. In addition, isolated electrons have two special requirements:

- the difference in the energy in a cone of $\Delta \mathrm{R}=0.2$ around the electron direction and the smeared electron energy needs to be below an energy threshold of $10 \mathrm{GeV}$,

- there should be no other clusters reconstructed within a cone of $\Delta R=0.4$ around the electron direction.

If the isolation criteria are passed, the reconstructed electron energy is obtained by smearing its true energy using the resolution functions based on results from test 
beams:

$$
\begin{aligned}
\frac{\sigma}{E}= & \frac{12 \% \sqrt{\mathrm{GeV}}}{\sqrt{E}} \oplus 7 \% \oplus \frac{0.245 \% \mathrm{GeV}}{E} \quad \text { for }|\eta| \leq 1.4 \\
\frac{\sigma}{E}= & \frac{12 \% \sqrt{\mathrm{GeV}}}{\sqrt{E}} \oplus 7 \% \oplus \frac{(0.36(2.3-|\eta|)+0.228) \% \mathrm{GeV}}{E} \\
& \text { for } 1.4<|\eta|<2.5,
\end{aligned}
$$

where the units of $E$ are $\mathrm{GeV}$.

Once the functions are applied, an isolated electron is recorded if its energy is above $5 \mathrm{GeV}$ and the true electron is located within $|\eta|<2.5$. The isolated electron is recorded with the $\eta$ and $\phi$ coordinates of the true electron and the associated calorimeter cluster is removed from the cluster list. Electrons are also reconstructed if no associated calorimeter cluster with $\Delta \mathrm{R}=0.15$ around the electron direction is found.

The default efficiency for lepton identification in ATLFAST is 100\%, but inefficiencies due to the isolation criteria arise. The efficiency for electrons of varying transverse momentum in LST1 was calculated to be approximately $80 \%$.

\section{Photons}

The isolation criteria for photons is similar to that of electrons, using the resolution functions of Equation (B.1) and Equation (B.3). For photons, however, a sampling term of $10 \%$ is used to account for the fact that photons are not affected by energy loss due to bremsstrahlung. The noise and constant terms remain the same in both cases.

In the ATLFAST simulation, there is no distinction between converted and unconverted photons and there is a smearing of the photon direction in $\eta$ according 
to the resolution function:

$$
\begin{array}{ll}
\sigma(\theta)=\frac{65 \mathrm{mrad} \sqrt{\mathrm{GeV}}}{\sqrt{E}} & \text { for }|\eta|<0.8, \\
\sigma(\theta)=\frac{50 \mathrm{mrad} \sqrt{\mathrm{GeV}}}{\sqrt{E}} & \text { for } 0.8 \leq|\eta|<1.4, \\
\sigma(\theta)=\frac{40 \mathrm{mrad} \sqrt{\mathrm{GeV}}}{\sqrt{E}} & \text { for }|\eta| \geq 1.4,
\end{array}
$$

where the units of $E$ are $\mathrm{GeV}$.

\section{Muons}

For each true muon with $p_{T}>0.5 \mathrm{GeV}$, the reconstructed momentum is calculated from the true momentum with a Gaussian resolution function depending on $p_{T}, \eta$ and $\phi$. After the resolution function is applied, the muon is accepted if $p_{T}>5 \mathrm{GeV}$ and it is within $|\eta|<2.5$.

The isolation criteria are very similar to that of electrons; however, the energy isolation cone is raised to $\Delta R=0.4$. Depending on the isolation criteria, the muons are classified as isolated or non-isolated muons. All muons that lie within $\Delta \mathrm{R}=0.4$ to a reconstructed jet are added to the jet such that the $p_{T}$ of the jet is changed. The efficiency for identifying isolated muons of varying transverse momentum in LST1 was calculated to be approximately $70 \%$.

\section{Jets}

All clusters that are not assigned to electrons or photons are considered as jets if their transverse energy exceeds an energy threshold of $10 \mathrm{GeV}$. The jet energy is taken to 
be the cluster energy including muons and is smeared according to the jet resolution

$$
\begin{aligned}
\frac{\sigma}{E}=\frac{50 \% \sqrt{\mathrm{GeV}}}{\sqrt{E}} \oplus 3 \%, & |\eta| \leq 3.2, \\
\frac{\sigma}{E}=\frac{100 \% \sqrt{\mathrm{GeV}}}{\sqrt{E}} \oplus 7 \%, & 3.2<|\eta|<4.9,
\end{aligned}
$$

where the units of $E$ are $\mathrm{GeV}$,

The direction of the jet is taken to be that of the cluster direction. Since the calorimeter response is set equal to one, jet calibrations are not needed to correct for lower and non-uniform calorimeter response to hadrons. However, out-of-cone corrections are needed and are applied at a separate jet calibration stage.

$\tau$

For each true hadronically decaying $\tau$, a $\tau$-labelling procedure is performed first, consisting of two requirements:

- matching of a true hadronically decaying $\tau$ within $\Delta \mathrm{R}<0.3$ to a reconstructed jet,

- ratio between the visible $\tau$ energy and the jet energy must be larger than

$$
1-\frac{2 \sigma\left(p_{T}^{J e t}\right)}{p_{T}^{J e t}},
$$

where $\sigma\left(p_{T}^{\text {Jet }}\right)$ is the expected $p_{T}$ resolution of the hadronic $\tau$ candidate.

If the $\tau$-labelling criteria are fulfilled, $\tau$ identification efficiencies are applied. These efficiencies are obtained using full simulation and are provided as a function of $p_{T}$ and $\eta$ in the form of a parameterization. 


\section{$b$-, c-Jets}

In the same way that $\tau$ 's are handled, $b$ - and $c$-jets go through a labeling step first. If a $b$-quark with $p_{T}>5 \mathrm{GeV}$ is found at the event generator level after final state radiation and a jet can be matched within $\Delta \mathrm{R}<0.2$, then the jet is called a $b$-labelled jet. This criteria is also true for $c$-jets.

As in the case of $\tau, b$ - and $c$-tagging is performed on a statistical basis using parameterizations. The default efficiency for $b$-tagging is $60 \%$.

\section{Missing $p_{T}$}

The missing transverse momentum $\left(\not \not_{T}\right)$ is calculated from reconstructed objects such as isolated electrons, photons, muons, $\tau$, jets, non-isolated muons and the remaining clusters not associated with any jets. Cells that are not associated with any clusters are also included in the calculation. The energies are smeared using the jet resolution function given in Equation (B.6) and Equation (B.7). 


\section{Appendix C}

\section{Invariant Mass Distribution}

\section{Formulas}

The formulas for the invariant mass distributions are derived in [53], following the decay chain illustrated in Figure 4.4. The fit parameters $a$ and $\left(M_{b c}^{\max }\right)^{2}$ are defined as in Equation (4.8) and Equation (4.10), with $M_{l c}^{\max }$ dependent on $M_{b c}^{\max }$ :

$$
\left(M_{l c}^{\max }\right)^{2}=\left(M_{b c}^{\max }\right)^{2} \frac{m_{t}^{2}}{m_{W}^{2}}
$$

With these values, the invariant mass distributions are:

$$
\frac{1}{\Gamma_{0}} \frac{\partial \Gamma}{\partial M_{b c}^{2}}=\frac{(1+a)}{2 a\left(M_{b c}^{\text {max }}\right)^{2}} \times\left\{\begin{array}{lr}
\ln \frac{1+a}{1-a}, & \text { for } 0<M_{b c}^{2}<\left(M_{b c}^{\max }\right)^{2} \frac{1-a}{1+a} \\
\ln \frac{\left(M_{b c}^{\max }\right)^{2}}{M_{b c}^{2}}, & \text { for }\left(M_{b c}^{\max }\right)^{2} \frac{1-a}{1+a}<M_{b c}^{2}<\left(M_{b c}^{\text {max }}\right)^{2}
\end{array}\right.
$$


and

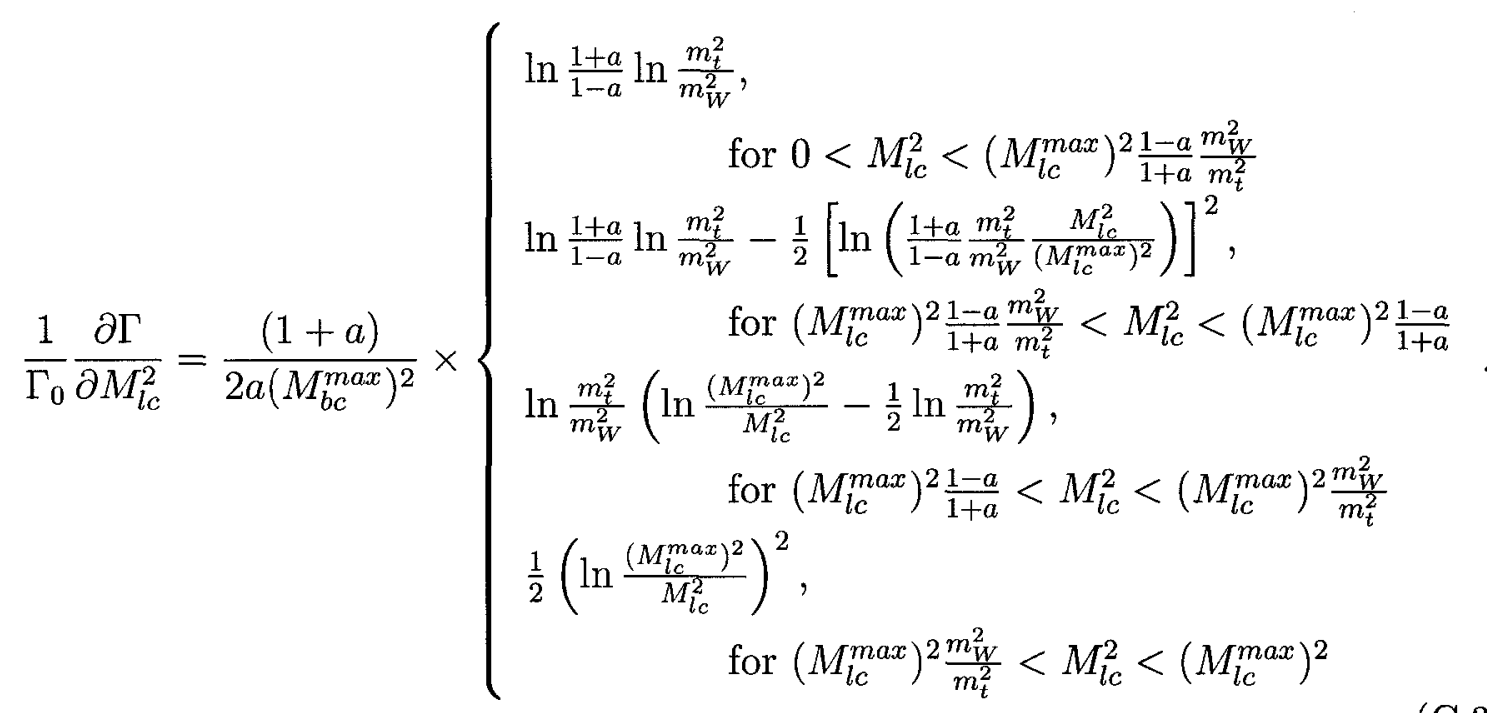

The values for $a$ and $M_{b c}^{\max }$ are then given by

$$
\begin{gathered}
a \equiv \frac{m_{2}^{2}}{m_{1}^{2}} \\
m_{1}^{2}=m_{\tilde{g}}^{2}-m_{t}^{2}-m_{\tilde{t}}^{2}, \quad m_{2}^{4}=m_{1}^{4}-4 m_{t}^{2} m_{\tilde{t}}^{2} \\
\left(M_{b c}^{\max }\right)^{2}=\frac{\left(m_{t}^{2}-m_{W}^{2}\right)}{m_{t}^{2}} \frac{\left(m_{\tilde{t}_{1}}^{2}-m_{\tilde{\chi}_{1}^{0}}^{2}\right)\left(m_{1}^{2}+m_{2}^{2}\right)}{2 m_{\tilde{t}_{1}}^{2}} .
\end{gathered}
$$

The nominal values for these parameters in the LST1 benchmark are $a=0.991$ and $M_{b c}^{\max }=392 \mathrm{GeV}$. 


\section{Appendix D}

\section{Projects}

During the course of my Ph.D. I have been included as an author on three publications; two ATLAS notes and one conference proceeding. I have also presented work at two major conferences and in over twenty ATLAS collaboration meetings. Below is a summary of the work in which I was involved.

I began my Ph.D. working on the T2K project, a long baseline neutrino oscillation experiment in Japan. My research focused on Research \& Development for one of the detectors involved. In particular, I used Monte Carlo simulation tools to investigate the properties of wavelength shifting fibers embedded into plastic scintillating tiles. As part of the project, I implemented the wavelength shifting process into Geant 4 . The results of the project were presented at a Geant4 Collaboration Workshop [87] as well as at the IEEE Nuclear Science Symposium and Medical Imaging Conference in 2003. The results are also published in the IEEE conference proceedings [88], but are not included in this thesis.

Over the course of four years on the ATLAS experiment, I have been involved 
in many projects. My work began on the 2004 beam test which investigated the combined performance of the electromagnetic end-cap calorimeter, the hadronic end-cap calorimeter and the forward calorimeter (FCal). As a beam shift worker, part of the responsibilities required the monitoring of beamline detectors as well as ensuring data quality in the calorimeters. Upon completion of data taking, analysis was performed on the pedestal levels of beam line scintillators which were used for triggering and event selection. The Liquid Argon Calorimeter Group meets approximately every three months in collaboration meetings referred to as "LAr Weeks" and the results of the scintillator studies were presented at the LAr Week in November, 2004 [89]. Because of this work, I am an author of the resulting publication, "Performance of the ATLAS Liquid Argon Endcap Calorimeter in the Pseudorapidity Region $2.5<|\eta|<$ 4.0 in Beam Tests" [90].

The next project focused on the Monte Carlo simulations of the Forward Calorimeter, in collaboration with Mohsen Khakzad, a Research Associate at Carleton University. As discussed in Section 3.3.3.1, complete energy deposition information is available in the Monte Carlo description of the ATLAS calorimeter system. The software required to accomplish the calculations was not complete for the FCal, and it was our goal to finish it. The results of this work were presented at the LAr Week in September, 2005 [91] as well as at the inaugural ATLAS Canada Workshop [92]. This naturally lead to the calculations of the sampling fractions for the FCal, also presented to the LAr collaboration [93]. Through the evolution of the ATLAS experiment, the sampling fraction calculations were performed for different versions of detector descriptions, versions of Geant 4 and versions of the ATLAS software framework (ATHENA) [94]. Working on the simulation of the calorimeter system, I am included as an author on the ATLAS note "The Simulation of the ATLAS Liquid 
Argon Calorimetry" [47] as well as on the ATLAS detector paper, "The ATLAS Experiment at the CERN Large Hadron Collider" [22].

My project then shifted to the Monte Carlo simulations of the 2003 beam test which investigated the performance of the FCal (Section 3.3). My role was to implement the beam test geometry into the ATLAS software framework. While the FCal geometry could be recycled from the complete ATLAS Monte Carlo geometry description, all of the beamline detector descriptions were required. In addition, the positioning of the FCal within the cryostat and the positioning of the cryostat was implemented for all the possible positions studied in the beam test. Configuration files were developed for all particle types, energies and FCal positions of the beam test. An analysis tool was developed within ATHENA for studies of the Monte Carlo performance. Analysis was then performed for both electron (Section 3.3.3) and pion Monte Carlo events, of which, only the electron studies are included in this thesis. Some of the electron results are included in the publication "Energy Calibration of the ATLAS Liquid Argon Forward Calorimeter" [45] of which I am an author, while the pion results were presented at an ATLAS Hadronic Calibration Workshop [52]. The electron results provided in the thesis were also presented at the 2008 Canadian Association of Physicists (CAP) Congress [95].

The last project was the investigation of a light supersymmetric partner to the top quark, in collaboration with M. Vincter and Zhaoyu Yang, a Research Associate at Carleton University. The results of our studies are shown in Chapter 4 and have been submitted as an ATLAS communication note [96]. As this was the first such analysis performed in the ATLAS collaboration, the Monte Carlo was completely generated at Carleton University; from simulation of the events to fast detector simulation (with the help of Yun-Ha Shin, Research Software Support) and analysis. The Monte Carlo 
samples have been validated and are included in the official ATLAS Monte Carlo studies (MC No.: 006443). Results of the studies have been presented to the ATLAS SUSY Working Group [97] as well as the at the ATLAS Canada Workshop in April, $2008[98]$. 


\section{Bibliography}

[1] J. C. Maxwell. A Dynamical Theory of the Electromagnetic Field. Phil. Trans. Roy. Soc. Lond., 155:459-512, 1865.

[2] S. L. Glashow. Nucl. Phys., 22:579, 1961.

[3] S. Weinberg. Phys. Rev. Lett., 19:1264, 1967.

[4] A. Salam. Weak and Electromagnetic Interactions, 1968. Originally printed in Svartholm: Elementary Particle Theory, Proceedings Of The Nobel Symposium Held 1968 At Lerum, Sweden.

[5] S. L. Glashow, Nucl. Phys. 22 (1961) 579, S. Weinberg, Phys. Rev. Lett. 19 (1967) 1264, A. Salam, In Elementary Particle Theory, ed. N. Svartholm, Stockholm, "Almquist and Wiksell" (1968), 367.

[6] C. Amsler et al. Review of Particle Physics. Phys. Lett., B667:1, 2008.

[7] Y. Fukuda et al. Evidence for oscillation of atmospheric neutrinos. Phys. Rev. Lett., 81:1562-1567, 1998.

[8] B. Aharmim et al. Electron Energy Spectra, Fluxes, and Day-Night Asymmetries of B-8 Solar Neutrinos from the 391-day Salt Phase SNO Data Set. Phys. Rev., C72:055502, 2005. 
[9] S. Tomonaga. On a Relativistically Invariant Formulation of the Quantum Theory of Wave Fields. Prog. Theor. Phys., 1:27-42, 1946.

[10] J. S. Schwinger. On Quantum Electrodynamics and the Magnetic Moment of the Electron. Phys. Rev., 73:416-417, 1948.

[11] R.P. Feynman. Space-time Approach to Quantum Electrodynamics. Phys. Rev., 76:769-789, 1949.

[12] F. J. Dyson. The Radiation Theories of Tomonaga, Schwinger, and Feynman. Phys. Rev., 75:486-502, 1949.

[13] D. J. Gross and F. Wilczek. Ultraviolet Behavior of Non-Abelian Gauge Theories. Phys. Rev. Lett., 30:1343-1346, 1973.

[14] H. D. Politzer. Reliable perturbative results for strong interactions? Phys. Rev. Lett., 30(26):1346-1349, 1973.

[15] P. W. Higgs. Broken Symmetries, Massless Particles and Gauge Fields. Phys. Lett., 12:132-133, 1964.

[16] F. Englert and R. Brout. Broken Symmetry and the Mass of Gauge Vector Mesons. Phys. Rev. Lett., 13:321-322, 1964.

[17] P. W. Higgs. Broken Symmetries and the Masses of Gauge Bosons. Phys. Rev. Lett., 13:508-509, 1964.

[18] R. Barate et al. Search for the Standard Model Higgs boson at LEP. Phys. Lett., B565:61-75, 2003. 
[19] C. L. Bennett et al. First Year Wilkinson Microwave Anisotropy Probe (WMAP) Observations: Preliminary Maps and Basic Results. Astrophys. J. Suppl., 148:1, 2003.

[20] D. N. Spergel et al. First Year Wilkinson Microwave Anisotropy Probe (WMAP) Observations: Determination of Cosmological Parameters. Astrophys. J. Suppl., 148:175, 2003.

[21] L. (ed.) Evans and P. (ed.) Bryant. LHC Machine. JINST, 3:S08001, 2008.

[22] G. Aad et al. The ATLAS Experiment at the CERN Large Hadron Collider. JINST, 3:S08003, 2008.

[23] ATLAS Inner Detector: Technical Design Report. CERN-LHCC-97-16, 1, 1997.

[24] ATLAS Inner Detector: Technical Design Report. CERN-LHCC-97-17, 1, 1997.

[25] ATLAS Tile Calorimeter: Technical Design Report. CERN-LHCC-96-42, 1996.

[26] ATLAS Liquid Argon Calorimeter: Technical Design Report. CERN-LHCC-9641, 1996.

[27] A. Airapetian et al. ATLAS Calorimeter Performance. CERN-LHCC-96-40, 1996.

[28] ATLAS Muon Spectrometer: Technical Design Report. CERN-LHCC-97-22, 1997.

[29] ATLAS First-Level Trigger: Technical Design Report. CERN-LHCC-98-14, 1998.

[30] ATLAS High-Level Trigger, Data Acquisition and Controls: Technical Design Report. CERN-LHCC-2003-022, 2003. 
[31] G. Duckeck et al. ATLAS Computing: Technical Design Report. CERN-LHCC2005-022, 2005.

[32] J. Boudreau and V. Tsulaia. The GeoModel Toolkit for Detector Description. 2004. Prepared for CHEP '04, Interlaken, Switzerland, September 27-October 1, 2004.

[33] S. Agostinelli et al. GEANT4: A Simulation Toolkit. Nucl. Instrum. Meth., A506:250-303, 2003.

[34] D. Cavalli et al. Performance of the ATLAS Fast Simulation ATLFAST. ATLPHYS-INT-2007-005, 2007.

[35] R. C. Fernow. Introduction to Experimental Particle Physics. Cambridge University Press, 1986.

[36] R. Wigmans. Calorimetry: Energy Measurement in Particle Physics. Int. Ser. Monogr. Phys., 107:1-726, 2000.

[37] R. Wigmans. On the Energy Resolution of Uranium and Other Hadron Calorimeters. Nucl. Instrum. Meth., A259:389-429, 1987.

[38] T. A. Gabriel et al. Energy Dependence of Hadronic Activity. Nucl. Instrum. Meth., A338:336-347, 1994.

[39] T. S. Virdee. Experimental Techniques. Prepared for 1998 European School of High-Energy Physics, St. Andrews, Scotland, 23 Aug - 5 Sep 1998, 1998.

[40] A. Artamonov et al. The ATLAS Forward Calorimeters. JINST, 3:P02010, 2008.

[41] J. Rutherfoord. Heating and Cooling a Forward Calorimeter. ATLAS Note ATL-CAL-94-056, 1994. 
[42] J. Rutherfoord. The Shape of the ATLAS Forward Calorimeter Current Pulse. ATL-LARG-PUB-2006-004, 2006.

[43] J. C. Armitage et al. Electron Signals in the Forward Calorimeter Prototype for ATLAS. JINST, 2:P11001, 2007.

[44] J. Rutherfoord. Signal Degradation due to Charge Buildup in Noble Liquid Ionization Calorimeters. Nucl. Instrum. Meth., A482:156-178, 2002.

[45] J. P. Archambault et al. Energy Calibration of the ATLAS Liquid Argon Forward Calorimeter. JINST, 3:P02002, 2008.

[46] http://www. rohacell.com/.

[47] J. P. Archambault et al. The Simulation of the ATLAS Liquid Argon Calorimetry. ATL-COM-LARG-2008-002, 2008.

[48] Geant4 Collaboration: Geant4 Physics Lists. http://www.geant4.org/geant4/ support/proc_mod_catalog/physics_lists/referencePL.shtml.

[49] M. P. Guthrie et al. Calculation of the Capture of Negative Pions in Light Elements and Comparison with Experiments Pertaining to Cancer Radiotherapy. Nucl. Instrum. Meth., 66:29-36, 1968.

[50] A. Heikkinen. Bertini Intra-nuclear Cascade Implementation in Geant4. 2003. nucl-th/0306008.

[51] H. W. Lewis. Multiple Scattering in an Infinite Medium. Phys. Rev., 78:526-529, 1950.

[52] J. P. Archambault. Studies of Hadrons with ATLAS Forward Calorimeter Monte Carlo. http://www.physics. arizona. edu/hcw2008/index.html. 
[53] S. Kraml and A. R. Raklev. Same-Sign Top Quarks as Signature of Light Stops at the LHC. Phys. Rev., D73:075002, 2006.

[54] H. E. Haber and G. L. Kane. The Search for Supersymmetry: Probing Physics Beyond the Standard Model. Phys. Rept., 117:75-263, 1985.

[55] S. P. Martin. A Supersymmetry Primer, 1997. hep-ph/9709356.

[56] Y. A. Golfand and E. P. Likhtman. Extension of the Algebra of Poincare Group Generators and Violation of P Invariance. JETP Lett., 13:323-326, 1971.

[57] P. Ramond. Dual Theory for Free Fermions. Phys. Rev., D3:2415-2418, 1971.

[58] A. Neveu and J. H. Schwarz. Factorizable Dual Model of Pions. Nucl. Phys., B31:86-112, 1971.

[59] D. V. Volkov and V. P. Akulov. Is the Neutrino a Goldstone Particle? Phys. Lett., B46:109-110, 1973.

[60] J. Wess and B. Zumino. Supergauge Transformations in Four-Dimensions. Nucl. Phys., B70:39-50, 1974.

[61] G. W. Anderson and D. J. Castano. Measures of Fine Tuning. Phys. Lett., B347:300-308, 1995.

[62] G. R. Farrar and P. Fayet. Phenomenology of the Production, Decay, and Detection of New Hadronic States Associated with Supersymmetry. Phys. Lett., B76:575-579, 1978.

[63] H. Goldberg. Constraint on the photino mass from cosmology. Phys. Rev. Lett., 50:1419, 1983. 
[64] J. R. Ellis et al. Supersymmetric Relics from the Big Bang. Nucl. Phys., B238:453-476, 1984.

[65] J. R. Ellis and S. Rudaz. Search for Supersymmetry in Toponium Decays. Phys. Lett., B128:248, 1983.

[66] A. Riotto and M. Trodden. Recent Progress in Baryogenesis. Ann. Rev. Nucl. Part. Sci., 49:35-75, 1999.

[67] A. D. Sakharov. Violation of CP Invariance, C Asymmetry, and Baryon Asymmetry of the Universe. Pisma Zh. Eksp. Teor. Fiz., 5:32-35, 1967.

[68] D. Delepine et al. A Light Stop and Electroweak Baryogenesis. Phys. Lett., B386:183-188, 1996.

[69] M. S. Carena et al. Electroweak Baryogenesis and Higgs and Stop searches at LEP and the Tevatron. Nucl. Phys., B524:3-22, 1998.

[70] C. Balazs et al. Dark Matter, Light Stops and Electroweak Baryogenesis. Phys. Rev., D70:015007, 2004.

[71] J. M. Cline et al. Supersymmetric Electroweak Phase Rransition: Baryogenesis versus Experimental Constraints. Phys. Rev. Lett., 81:3315-3318, 1998.

[72] A. Heister et al. Search for Scalar Quarks in e+ e- Collisions at $\mathrm{s}^{* *}(1 / 2)$ up to 209-GeV. Phys. Lett., B537:5-20, 2002.

[73] J. Abdallah et al. Searches for Supersymmetric Particles in e+ e- collisions up to 208-GeV and Interpretation of the Results within the MSSM. Eur. Phys. J., C31:421-479, 2004. 
[74] P. Achard et al. Search for Scalar Leptons and Scalar Quarks at LEP. Phys. Lett., B580:37-49, 2004.

[75] V. M. Abazov et al. Search for Pair Production of Light Scalar Top Quarks in $p \bar{p}$ Collisions at $\sqrt{s}=1.8 \mathrm{TeV}$. Phys. Rev. Lett., 93:011801, 2004.

[76] A. A. Affolder et al. Search for Scalar Top and Scalar Bottom Quarks in $p \bar{p}$ Collisions at $\sqrt{s}=1.8 \mathrm{TeV}$. Phys. Rev. Lett., 84:5704-5709, 2000.

[77] W. M. Yao et al. Review of Particle Physics. J. Phys., G33:1-1232, 2006.

[78] F. E. Paige et al. ISAJET 7.69: A Monte Carlo Event Generator for p p, anti-p p, and e+ e- Reactions, 2003. hep-ph/0312045.

[79] G. Marchesini et al. HERWIG: A Monte Carlo Event Generator for Simulating Hadron Emission Reactions with Interfering Gluons. Comput. Phys. Commun., 67:465-508, 1992. Version 5.1.

[80] G. Corcella et al. HERWIG 6: An Event Generator for Hadron Emission Reactions with Interfering Gluons (including supersymmetric processes). JHEP, 01:010, 2001.

[81] G. Corcella et al. HERWIG 6.5 Release Note. 2002. hep-ph/0210213.

[82] S. Moretti et al. Implementation of Supersymmetric Processes in the HERWIG Event Generator. JHEP, 04:028, 2002.

[83] ATLAS SUSY Working Group. ATLFAST Background. https://twiki.cern. $\mathrm{ch} /$ twiki/bin/view/AtlasProtected/AtlfastProduction.

[84] ATLAS Detector and Physics Performance. Technical Design Report. CERNLHCC-99-15, 2. 
[85] J. Pumplin et al. New Generation of Parton Distributions with Uncertainties from Global QCD Analysis. JHEP, 07:012, 2002.

[86] ATLAS Collaboration. Expected Performance of the ATLAS Experiment. 2008. CERN-OPEN-2008-020.

[87] J. P. Archambault and P. Gumplinger. Geant4 Photon Readout Simulations of Plastic Scintillating Strips with Embedded WLS Fibers. September, 2003. Geant4 Collaboration Workshop.

[88] J. P. Archambault et al. Geant4 Photon Readout Simulations of Plastic Scintillating Strips with Embedded WLS Fibers. 2003. Conference proceedings of IEEE Nuclear Science Symposium and Medical Imaging Conference.

[89] J. P. Archambault and M. G. Vincter. Pedestal Study of Scintillators. http: //indico. cern.ch/conferenceDisplay. py?confId=a044995.

[90] J. Pinfold et al. Performance of the ATLAS Liquid Argon Endcap Calorimeter in the Pseudorapidity Region $2.5<\mid$ eta $\mid<4.0$ in Beam Tests. Nucl. Instrum. Meth., A593:324-342, 2008.

[91] J. P. Archambault and M. Khakzad. G4 ATLAS FCal Calibration Hits. http: //indico.cern. ch/conferenceDisplay. py? conf Id=a055700.

[92] J. P. Archambault and M. Khakzad. MC Definitions: Standard and Calibration Hits. http://indico. cern. ch/conferenceDisplay. py? conf Id=a056950.

[93] J. P. Archambault and M. Khakzad. Update on FCAL Sampling Fraction. http: //indico.cern.ch/conferenceDisplay.py? conf Id=a063003.

[94] P. Krieger. FCal Simulation and Performance Summary. http://indico.cern. ch/conferenceDisplay $\cdot$ py? conf Id $=13514$. 
[95] J. P. Archambault. Geant4 Studies of the ATLAS Forward Calorimeter. June 2008. Presented at Canadian Association of Physicists (CAP) Congress.

[96] J. P. Archambault, M. Vincter, and Z. Yang. Light Stop Searches with the ATLAS Detector. ATL-COM-PHYS-2009-091, 2009.

[97] J. P. Archambault et al. Search for a Light Stop with Same-Sign Leptons. http: //indico.cern. ch/conferenceDisplay.py? conf Id=31736.

[98] J. P. Archambault et al. Search for a Light Stop using SameSign Top Quarks. http://indico.cern.ch/conferenceOtherViews.py?view= standard\&conf Id=15084. 